\title{
Synthesis of Tetrasubstituted Pyrroles from Terminal Alkynes and Imines
}

\section{Supporting Information}

Yancheng Hu, Chunxiang Wang, Dongping Wang, Fan Wu, and Boshun Wan*

Dalian Institute of Chemical Physics, Chinese Academy of Sciences, 457 Zhongshan Road, Dalian 116023, China

bswan@dicp.ac.cn 


\section{Table of contents}

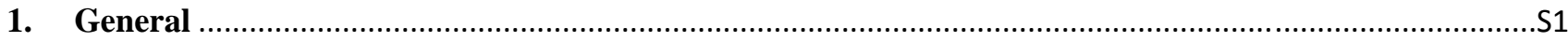

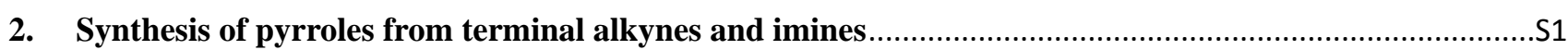

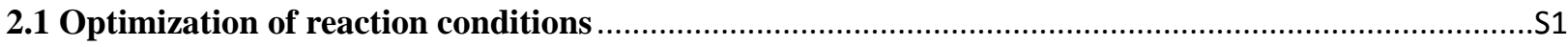

2.2 Representative procedures for the synthesis of pyrroles from terminal alkynes and imines..............S1

3. Synthesis of tetrasubstituted pyrroles from propargylamines and imines ............................................ 8

3.1 General procedures for the synthesis of propargylamines ...................................................

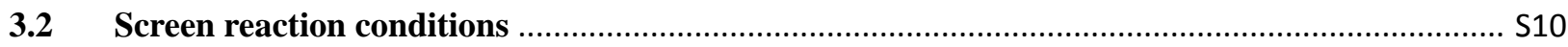

3.3 Typical procedures for the synthesis of pyrroles from propargylamines and imines ................ S10

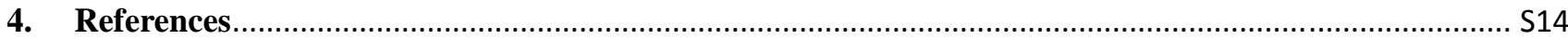

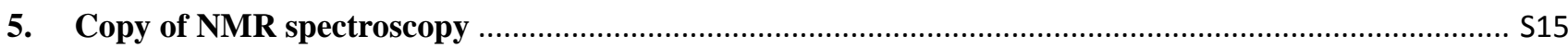




\section{General}

Unless otherwise noted, all reactions and manipulations were carried out under an atmosphere of argon using standard Schlenk techniques or in an argon-filled glove-box. Solvents were treated prior to use according to the standard methods. All chemicals were obtained from commercial sources and were used without further purification. Column chromatography was carried out on silica gel (300-400 mesh) using a forced flow of eluent at $0.3-0.5$ bar pressure. For TLC, silica gel GF254 was used and visualized by fluorescence quenching under UV light.

NMR Spectra were recorded on a Bruker $400 \mathrm{MHz}$ or $500 \mathrm{MHz}$ NMR spectrometer in the solvents indicated. The chemical shifts for ${ }^{1} \mathrm{H}$ NMR were recorded in ppm downfield from tetramethylsilane (TMS) with $\mathrm{CDCl}_{3}(7.26$ ppm) as the internal standard. The chemical shifts for ${ }^{13} \mathrm{C}$ NMR were recorded in ppm downfield using the central peak of $\mathrm{CDCl}_{3}(77.16 \mathrm{ppm})$ as the internal standard. Coupling constants (J) are reported in hertz and refer to apparent peak multiplications. Imines were obtained from the corresponding aldehyde and amine, according to the published literature ${ }^{1}$. And the propargylamines were synthesized by the reported method ${ }^{2}$.

\section{Synthesis of pyrroles from terminal alkynes and imines}

\subsection{Optimization of reaction conditions ${ }^{a}$}

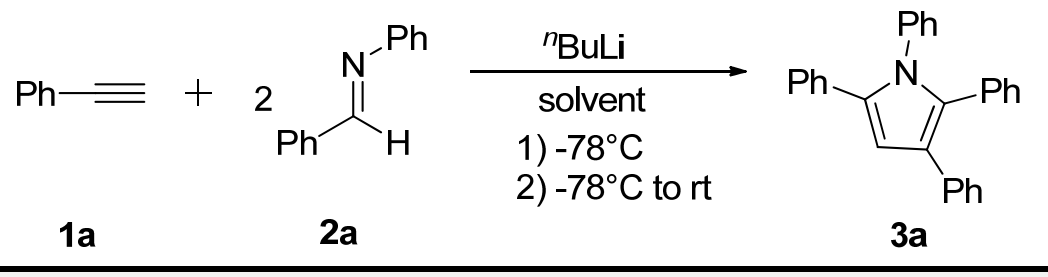

\begin{tabular}{cccc}
\hline Entry & Solvent & ${ }^{n}$ BuLi (equiv) & Yield (\%) ${ }^{b}$ \\
\hline 1 & THF & 1 & 83 \\
2 & THF & 2 & 45 \\
3 & THF & 3 & 0 \\
4 & $\mathrm{Et}_{2} \mathrm{O}$ & 1 & trace \\
5 & toluene & 1 & 0 \\
6 & PhOMe & 1 & 0 \\
${ }^{a} 0.625$ mmol 1a, 1.25 mmol 2a, 4 mL solvent, $-78^{\circ} \mathrm{C}$ for $1 \mathrm{~h}, \mathrm{rt}$ for $10 \mathrm{~h}$. \\
${ }^{b}$ Determined by HPLC. Naphthalene was used as internal standard. \\
\hline
\end{tabular}

\subsection{Representative procedures for the synthesis of pyrroles from terminal alkynes and imines}

2.5 M BuLi in hexane $(0.25 \mathrm{~mL}, 0.625 \mathrm{mmol})$ was added dropwise with stirring to a solution of terminal alkyne 1 $(0.625 \mathrm{mmol})$ in THF $(2 \mathrm{~mL})$ at $-78{ }^{\circ} \mathrm{C}$. The mixture was stirred for $45-60 \mathrm{~min}$, and subsequently, an appropriate imine $2(1.25 \mathrm{mmol})$ in THF $(2 \mathrm{~mL})$ was added dropwise. The solution was stirred at room temperature for $10 \mathrm{~h}$, quenched with an aqueous saturated solution of ammonium chloride. The aqueous layer was extracted with $\mathrm{Et}_{2} \mathrm{O}$ and the combined organic layers were washed twice with brine, dried over $\mathrm{MgSO}_{4}$, filtered and concentrated under reduced pressure to give crude products. Purification by crystallization from EtOH/ pentane gave the desired pyrrole 3. 
<smiles>c1ccc(-c2cc(-c3ccccc3)n(-c3ccccc3)c2-c2ccccc2)cc1</smiles>

$3 a$

\section{1,2,3,5-tetraphenyl-1H-pyrrole}

White solid (81\% yield), m.p. $199-200{ }^{\circ} \mathrm{C}$

${ }^{1} \mathrm{H}$ NMR $\left(400 \mathrm{MHz}, \mathrm{CDCl}_{3}\right) \delta 7.17(\mathrm{~m}, 16 \mathrm{H}), 7.04(\mathrm{~d}, \mathrm{~J}=7.1 \mathrm{~Hz}, 2 \mathrm{H}), 7.01-6.94(\mathrm{~m}, 2 \mathrm{H}), 6.71(\mathrm{~s}, 1 \mathrm{H}) .{ }^{13} \mathrm{C} \mathrm{NMR}$ $\left(100 \mathrm{MHz}, \mathrm{CDCl}_{3}\right) \delta 138.9,136.3,135.0,133.1,132.8,132.3,131.6,129.3,128.7,128.6,128.3,128.1,128.0$, $127.3,127.1,126.5,125.7,123.6,110.2$.

HRMS calcd for $\mathrm{C}_{28} \mathrm{H}_{22} \mathrm{~N}[\mathrm{M}+\mathrm{H}]^{+} 372.1752$, found 372.1742<smiles>COc1ccc(-c2c(-c3ccccc3)cc(-c3ccccc3)n2-c2ccccc2)cc1</smiles>

\section{2,5-bis(4-methoxyphenyl)-1,3-diphenyl-1H-pyrrole}

Light yellow solid (62\% yield), m.p. $187-188^{\circ} \mathrm{C}$

${ }^{1} \mathrm{H}$ NMR $\left(500 \mathrm{MHz}, \mathrm{CDCl}_{3}\right) \delta 7.27-7.24(\mathrm{~m}, 2 \mathrm{H}), 7.23-7.15(\mathrm{~m}, 5 \mathrm{H}), 7.14-7.08(\mathrm{~m}, 1 \mathrm{H}), 7.06-7.00(\mathrm{~m}, 2 \mathrm{H})$, $6.99-6.91(\mathrm{~m}, 4 \mathrm{H}), 6.74-6.70(\mathrm{~m}, 2 \mathrm{H}), 6.69-6.65(\mathrm{~m}, 2 \mathrm{H}), 6.62(\mathrm{~s}, 1 \mathrm{H}), 3.75(\mathrm{~s}, 3 \mathrm{H}), 3.74(\mathrm{~s}, 3 \mathrm{H}) .{ }^{13} \mathrm{C} \mathrm{NMR}$ $\left(100 \mathrm{MHz}, \mathrm{CDCl}_{3}\right) \delta 158.6,158.3,139.1,136.5,134.5,132.7,131.6,129.9,129.3,128.5,128.2,127.1,125.8$, $125.4,125.3,123.0,113.6,113.5,109.1,55.3,55.2$.

HRMS (EI, $\mathrm{m} / \mathrm{z}$ ) calcd for $\mathrm{C}_{30} \mathrm{H}_{25} \mathrm{NO}_{2}[\mathrm{M}]^{+} 431.1885$, found 431.1880<smiles></smiles>

\section{2,5-bis(4-isopropylphenyl)-1,3-diphenyl-1H-pyrrole}

White solid (91\% yield), m.p. $199-200^{\circ} \mathrm{C}$

${ }^{1} \mathrm{H}$ NMR (400 MHz, CDCl $)_{3}$ ) $7.26-7.07$ (m, 8H), 7.03 (s, 4H), $6.95(\mathrm{dd}, \mathrm{J}=15.1,8.0 \mathrm{~Hz}, 6 \mathrm{H}), 6.66(\mathrm{~s}, 1 \mathrm{H}), 2.82$ $(\mathrm{dd}, \mathrm{J}=14.0,7.0 \mathrm{~Hz}, 2 \mathrm{H}), 1.19(\mathrm{t}, \mathrm{J}=7.7 \mathrm{~Hz}, 12 \mathrm{H}) .{ }^{13} \mathrm{C} \mathrm{NMR}\left(100 \mathrm{MHz}, \mathrm{CDCl}_{3}\right) \delta 147.6,146.9,139.2,136.5$, $134.8,132.2$, 131.4, 130.6, 130.1, 129.3, 128.5, 128.4, 128.3, 128.2, 127.0, 126.1, 125.9, 125.4, 123.2, 109.7, 33.8, 24.0 .

HRMS (EI, m/z) calcd for $\mathrm{C}_{34} \mathrm{H}_{33} \mathrm{~N}[\mathrm{M}]^{+} 455.2613$, found 455.2614 
<smiles></smiles>

3d

\section{2,5-bis(4-fluorophenyl)-1,3-diphenyl-1H-pyrrole}

Light yellow solid (70\% yield), m.p. $168-169^{\circ} \mathrm{C}$

${ }^{1} \mathrm{H}$ NMR $\left(500 \mathrm{MHz}, \mathrm{CDCl}_{3}\right) \delta 7.24-7.12(\mathrm{~m}, 8 \mathrm{H}), 7.10-7.04(\mathrm{~m}, 2 \mathrm{H}), 7.02-6.92(\mathrm{~m}, 4 \mathrm{H}), 6.90-6.80(\mathrm{~m}, 4 \mathrm{H})$, $6.65(\mathrm{~s}, 1 \mathrm{H}) .{ }^{13} \mathrm{C}$ NMR $\left(126 \mathrm{MHz}, \mathrm{CDCl}_{3}\right) \delta 161.9(\mathrm{~d}, \mathrm{~J}=247.1 \mathrm{~Hz}), 161.7(\mathrm{~d}, \mathrm{~J}=246.4 \mathrm{~Hz}), 138.5,135.9,134.0$, $133.1(\mathrm{~d}, \mathrm{~J}=8.1 \mathrm{~Hz}), 131.0,130.3,130.2(\mathrm{~d}, \mathrm{~J}=7.9 \mathrm{~Hz}), 129.1,129.0,128.7,128.2,127.4,125.7,123.7,115.03(\mathrm{~d}$, $J=21.4 \mathrm{~Hz}), 115.00(\mathrm{~d}, J=21.5 \mathrm{~Hz}), 109.8$.

${ }^{19} \mathrm{~F}$ NMR $\left(377 \mathrm{MHz}, \mathrm{CDCl}_{3}\right) \delta-115.1,-116.1$.

HRMS (EI, $\mathrm{m} / \mathrm{z}$ ) calcd for $\mathrm{C}_{28} \mathrm{H}_{19} \mathrm{~F}_{2} \mathrm{~N}[\mathrm{M}]^{+} 407.1486$, found 407.1485<smiles>Brc1cccc(-c2c(-c3ccccc3)cc(-c3ccccc3)n2-c2ccccc2)c1</smiles>

\section{2,5-bis(3-bromophenyl)-1,3-diphenyl-1H-pyrrole}

White solid (80\% yield), m.p. $151-152{ }^{\circ} \mathrm{C}$

${ }^{1} \mathrm{H}$ NMR (400 MHz, CDCl $) \delta 7.32(\mathrm{~s}, 1 \mathrm{H}), 7.29-7.20(\mathrm{~m}, 9 \mathrm{H}), 7.19-7.13(\mathrm{~m}, 2 \mathrm{H}), 6.97(\mathrm{qd}, J=14.3,7.8 \mathrm{~Hz}$, $6 \mathrm{H}), 6.70(\mathrm{~s}, 1 \mathrm{H}) .{ }^{13} \mathrm{C} \mathrm{NMR}\left(100 \mathrm{MHz}, \mathrm{CDCl}_{3}\right) \delta 138.3,135.5,134.8,134.6,134.2,133.8,131.4,131.1,130.3$, $130.1,129.5,129.1,129.0,128.4,128.3,127.9,127.0,126.1,124.5,122.2,121.9,110.9$.

HRMS (EI, m/z) calcd for $\mathrm{C}_{28} \mathrm{H}_{19} \mathrm{Br}_{2} \mathrm{~N}[\mathrm{M}]^{+}$526.9884, found 526.9883<smiles>c1ccc(-c2c([Bi]c3ccco3)cc(-c3ccco3)n2-c2ccccc2)cc1</smiles>

\section{2,5-di(furan-2-yl)-1,3-diphenyl-1H-pyrrole}

Light yellow solid (48\% yield), m.p. $114-115^{\circ} \mathrm{C}$

${ }^{1} \mathrm{H}$ NMR (400 MHz, CDCl $) \delta 7.41-7.33(\mathrm{~m}, 5 \mathrm{H}), 7.29$ (q, $\left.J=6.0 \mathrm{~Hz}, 5 \mathrm{H}\right), 7.24(\mathrm{~d}, J=0.9 \mathrm{~Hz}, 1 \mathrm{H}), 7.20(\mathrm{t}, J=$ $7.2 \mathrm{~Hz}, 1 \mathrm{H}), 6.86(\mathrm{~s}, 1 \mathrm{H}), 6.19(\mathrm{ddd}, J=5.9,3.3,1.8 \mathrm{~Hz}, 2 \mathrm{H}), 5.93-5.86(\mathrm{~m}, 1 \mathrm{H}), 5.28(\mathrm{~d}, J=3.3 \mathrm{~Hz}, 1 \mathrm{H}) .{ }^{13} \mathrm{C}$ NMR $\left(100 \mathrm{MHz}, \mathrm{CDCl}_{3}\right) \delta 147.2,145.6,142.3,141.2,139.1,135.6,129.0,128.7,128.3,128.0,127.7,126.5,126.3$, $122.9,111.4,111.0,110.8,108.5,105.5$.

HRMS (EI, m/z) calcd for $\mathrm{C}_{24} \mathrm{H}_{17} \mathrm{NO}_{2}[\mathrm{M}]^{+} 351.1259$, found 351.1249 
<smiles>c1ccc(-c2cc(-c3cccs3)n(-c3ccccc3)c2-c2ccccc2)cc1</smiles>

$3 g$

\section{1,3-diphenyl-2,5-di(thiophen-2-yl)-1H-pyrrole}

Light yellow solid (59\% yield), m.p. $165-166^{\circ} \mathrm{C}$

${ }^{1} \mathrm{H}$ NMR (500 MHz, $\mathrm{CDCl}_{3}$ ) $\delta 7.36-7.29(\mathrm{~m}, 5 \mathrm{H}), 7.26-7.21(\mathrm{~m}, 4 \mathrm{H}), 7.20-7.14(\mathrm{~m}, 2 \mathrm{H}), 7.07$ (dd, J= 5.1, 1.1 $\mathrm{Hz}, 1 \mathrm{H}), 6.81(\mathrm{ddd}, J=8.3,5.1,3.6 \mathrm{~Hz}, 2 \mathrm{H}), 6.74(\mathrm{~s}, 1 \mathrm{H}), 6.69(\mathrm{dd}, J=3.6,1.2 \mathrm{~Hz}, 1 \mathrm{H}), 6.55(\mathrm{dd}, J=3.6,1.1 \mathrm{~Hz}$, 1H). ${ }^{13} \mathrm{C} \mathrm{NMR}\left(100 \mathrm{MHz}, \mathrm{CDCl}_{3}\right) \delta 138.4,135.7,134.9,133.4,129.9,129.7,128.9,128.6,128.3,127.1,126.7$, $126.1,125.8,125.3,124.8,124.4,109.9$.

HRMS (EI, m/z) calcd for $\mathrm{C}_{24} \mathrm{H}_{17} \mathrm{NS}_{2}[\mathrm{M}]^{+} 383.0802$, found 383.0795<smiles>c1ccc(-c2cc(-c3ccc4ccccc4c3)c(-c3ccc4ccccc4c3)[nH]2)cc1</smiles>

\section{2,5-di(naphthalen-2-yl)-1,3-diphenyl-1H-pyrrole}

Light yellow solid ( $82 \%$ yield), m.p. $234-235{ }^{\circ} \mathrm{C}$

${ }^{1} \mathrm{H} \mathrm{NMR}\left(500 \mathrm{MHz}, \mathrm{CDCl}_{3}\right) \delta 7.74(\mathrm{~d}, J=6.9 \mathrm{~Hz}, 2 \mathrm{H}), 7.66-7.51(\mathrm{~m}, 6 \mathrm{H}), 7.45-7.34(\mathrm{~m}, 4 \mathrm{H}), 7.33-7.27(\mathrm{~m}$, $2 \mathrm{H}), 7.24-7.10(\mathrm{~m}, 8 \mathrm{H}), 7.09-7.05(\mathrm{~m}, 2 \mathrm{H}), 6.87(\mathrm{~s}, 1 \mathrm{H}) .{ }^{13} \mathrm{C} \mathrm{NMR}\left(100 \mathrm{MHz}, \mathrm{CDCl}_{3}\right) \delta 139.0,136.2,135.2$, $133.4,133.2,132.4,132.1,130.8,130.5,130.3,129.4,129.3,128.8,128.4,128.2,128.1,127.7,127.5,127.4,127.3$, $127.0,126.2,126.1,126.0,125.9,125.8,124.3,110.9$.

HRMS (EI, m/z) calcd for $\mathrm{C}_{36} \mathrm{H}_{25} \mathrm{~N}[\mathrm{M}]^{+}$471.1987, found 471.1980<smiles>Brc1ccc(-n2c(-c3ccccc3)cc(-c3ccccc3)c2-c2ccccc2)cc1</smiles>

$3 \mathbf{i}$

\section{1-(4-bromophenyl)-2,3,5-triphenyl-1H-pyrrole}

Light yellow solid (89\% yield), m.p. $165-166^{\circ} \mathrm{C}$

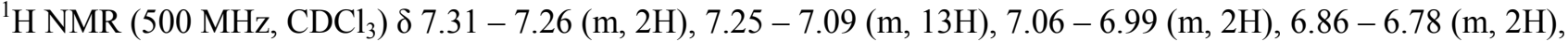
$6.69(\mathrm{~s}, 1 \mathrm{H}) .{ }^{13} \mathrm{C} \mathrm{NMR}\left(100 \mathrm{MHz}, \mathrm{CDCl}_{3}\right) \delta 138.0,136.0,134.9,132.7,132.5,132.1,131.8,131.6,130.7,128.8$, $128.3,128.2,127.4,126.7,125.8,124.0,121.0,110.5$.

HRMS (EI, m/z) calcd for $\mathrm{C}_{28} \mathrm{H}_{20} \mathrm{BrN}[\mathrm{M}]^{+} 449.0779$, found 449.0771 
<smiles>COc1ccc(-n2c(-c3ccccc3)cc(-c3ccccc3)c2-c2ccccc2)cc1</smiles>

\section{1-(4-methoxyphenyl)-2,3,5-triphenyl-1H-pyrrole}

Light yellow solid (62\% yield), m.p. $154-155^{\circ} \mathrm{C}$

${ }^{1} \mathrm{H}$ NMR $\left(400 \mathrm{MHz}, \mathrm{CDCl}_{3}\right) \delta 7.34-7.29(\mathrm{~m}, 2 \mathrm{H}), 7.27-7.14(\mathrm{~m}, 11 \mathrm{H}), 7.12(\mathrm{dt}, J=7.7,3.9 \mathrm{~Hz}, 2 \mathrm{H}), 6.99-6.92$ $(\mathrm{m}, 2 \mathrm{H}), 6.76(\mathrm{~s}, 1 \mathrm{H}), 6.74-6.69(\mathrm{~m}, 2 \mathrm{H}), 3.75(\mathrm{~s}, 3 \mathrm{H}) .{ }^{13} \mathrm{C} \mathrm{NMR}\left(100 \mathrm{MHz}, \mathrm{CDCl}_{3}\right) \delta 158.5,136.4,135.1,133.2$, 133.0, 132.6, 131.9, 131.7, 130.2, 128.7, 128.3, 128.2, 128.0, 127.1, 126.4, 125.6, 123.5, 113.8, 109.9, 55.4. HRMS (EI, m/z) calcd for $\mathrm{C}_{29} \mathrm{H}_{23} \mathrm{NO}[\mathrm{M}]^{+} 401.1780$, found 401.1771<smiles>Cc1cccc(-n2c(-c3ccccc3)cc(-c3ccccc3)c2-c2ccccc2)c1</smiles>

3k

\section{2,3,5-triphenyl-1-(m-tolyl)-1H-pyrrole}

Light yellow solid (84\% yield), m.p. $160-161{ }^{\circ} \mathrm{C}$

${ }^{1} \mathrm{H}$ NMR (500 MHz, $\left.\mathrm{CDCl}_{3}\right) \delta 7.28-7.23(\mathrm{~m}, 2 \mathrm{H}), 7.23-7.10(\mathrm{~m}, 11 \mathrm{H}), 7.07-7.01(\mathrm{~m}, 3 \mathrm{H}), 6.98(\mathrm{~d}, J=7.6 \mathrm{~Hz}$, $1 \mathrm{H}), 6.83-6.74(\mathrm{~m}, 2 \mathrm{H}), 6.70(\mathrm{~s}, 1 \mathrm{H}), 2.15(\mathrm{~s}, 3 \mathrm{H}) .{ }^{13} \mathrm{C} \mathrm{NMR}\left(100 \mathrm{MHz}, \mathrm{CDCl}_{3}\right) \delta 138.8,138.5,136.4,134.9$, 133.2, 132.9, 132.4, 131.6, 129.9, 128.6, 128.3, 128.1, 128.0, 127.9, 127.1, 126.4, 126.3, 125.6, 123.6, 110.1, 21.3. HRMS (EI, m/z) calcd for $\mathrm{C}_{29} \mathrm{H}_{23} \mathrm{~N}[\mathrm{M}]^{+} 385.1830$, found 385.1827<smiles>Cc1ccc(-c2cc(-c3ccccc3)n(-c3ccccc3)c2-c2ccccc2)cc1</smiles>

\section{1,2,5-triphenyl-3-(p-tolyl)-1H-pyrrole}

White solid (81\% yield), m.p. $157-158^{\circ} \mathrm{C}$

${ }^{1} \mathrm{H}$ NMR $\left(500 \mathrm{MHz}, \mathrm{CDCl}_{3}\right) \delta 7.20-7.10(\mathrm{~m}, 13 \mathrm{H}), 7.07-7.01(\mathrm{~m}, 4 \mathrm{H}), 7.01-6.96(\mathrm{~m}, 2 \mathrm{H}), 6.70(\mathrm{~s}, 1 \mathrm{H}), 2.31(\mathrm{~s}$, $3 \mathrm{H}) .{ }^{13} \mathrm{C}$ NMR $\left(126 \mathrm{MHz}, \mathrm{CDCl}_{3}\right) \delta 139.1,135.2,134.9,133.4,133.2,133.0,132.2,131.7,129.3,129.1,128.8$, 128.6, 128.2, 128.1, 128.0, 127.2, 127.1, 126.5, 123.6, 110.2, 21.3.

HRMS (EI, $\mathrm{m} / \mathrm{z}$ ) calcd for $\mathrm{C}_{29} \mathrm{H}_{23} \mathrm{~N}[\mathrm{M}]^{+} 385.1830$, found 385.1835 


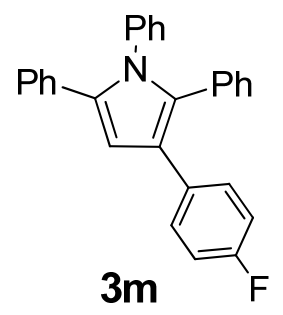

\section{3-(4-fluorophenyl)-1,2,5-triphenyl-1H-pyrrole}

Light yellow solid (80\% yield), m.p. $160-161{ }^{\circ} \mathrm{C}$

${ }^{1} \mathrm{H}$ NMR $\left(500 \mathrm{MHz}, \mathrm{CDCl}_{3}\right) \delta 7.22-7.08(\mathrm{~m}, 12 \mathrm{H}), 7.04-7.00(\mathrm{~m}, 2 \mathrm{H}), 6.99-6.95(\mathrm{~m}, 2 \mathrm{H}), 6.93-6.85(\mathrm{~m}, 2 \mathrm{H})$, $6.65(\mathrm{~s}, 1 \mathrm{H}) .{ }^{13} \mathrm{C}$ NMR $\left(100 \mathrm{MHz}, \mathrm{CDCl}_{3}\right) \delta 161.3(\mathrm{~d}, J=244.4 \mathrm{~Hz}), 138.9,135.0,133.0,132.7,132.3,131.6$, $129.8,129.7$ (d, $J=7.7 \mathrm{~Hz}), 129.2,128.7,128.1,127.3,127.2,126.6,122.7,115.1$ (d, $J=21.2 \mathrm{~Hz}), 110.0$.

${ }^{19} \mathrm{~F} \mathrm{NMR}\left(377 \mathrm{MHz}, \mathrm{CDCl}_{3}\right) \delta-118.2$.

HRMS (EI, $\mathrm{m} / \mathrm{z}$ ) calcd for $\mathrm{C}_{28} \mathrm{H}_{20} \mathrm{FN}[\mathrm{M}]^{+}$389.1580, found 389.1575<smiles>c1ccc(-c2cc(C3CC3)c(-c3ccccc3)n2-c2ccccc2)cc1</smiles>

\section{3-cyclopropyl-1,2,5-triphenyl-1H-pyrrole}

Light yellow solid (73\% yield), m.p. $171-172{ }^{\circ} \mathrm{C}$

${ }^{1} \mathrm{H}$ NMR $\left(500 \mathrm{MHz}, \mathrm{CDCl}_{3}\right) \delta 7.22-7.08(\mathrm{~m}, 11 \mathrm{H}), 7.06-7.01(\mathrm{~m}, 2 \mathrm{H}), 6.96-6.91(\mathrm{~m}, 2 \mathrm{H}), 6.09(\mathrm{~s}, 1 \mathrm{H}), 1.81(\mathrm{tt}$, $J=8.4,5.2 \mathrm{~Hz}, 1 \mathrm{H}), 0.84(\mathrm{ddd}, J=8.4,6.2,4.1 \mathrm{~Hz}, 2 \mathrm{H}), 0.72-0.60(\mathrm{~m}, 2 \mathrm{H}) .{ }^{13} \mathrm{C} \mathrm{NMR}\left(100 \mathrm{MHz}, \mathrm{CDCl}_{3}\right) \delta$ 139.1, 134.3, 133.3, 133.0, 132.8, 131.0, 129.0, 128.6, 128.0, 127.7, 126.9, 126.4, 126.2, 125.3, 106.3, 8.5, 7.9. HRMS (EI, m/z) calcd for $\mathrm{C}_{25} \mathrm{H}_{21} \mathrm{~N}[\mathrm{M}]^{+} 335.1674$, found 335.1667<smiles>CS(=O)(=O)c1cc(-c2ccccc2)n(-c2ccccc2)c1-c1ccccc1</smiles>

\section{1,2,5-triphenyl-3-(trimethylsilyl)-1H-pyrrole}

White solid (78\% yield), m.p. $142-143{ }^{\circ} \mathrm{C}$

${ }^{1} \mathrm{H}$ NMR (400 MHz, $\left.\mathrm{CDCl}_{3}\right) \delta 7.22-7.08(\mathrm{~m}, 13 \mathrm{H}), 7.02-6.89(\mathrm{~m}, 2 \mathrm{H}), 6.57(\mathrm{~s}, 1 \mathrm{H}), 0.10(\mathrm{~s}, 9 \mathrm{H}) .{ }^{13} \mathrm{C} \mathrm{NMR}(100$ $\left.\mathrm{MHz}, \mathrm{CDCl}_{3}\right) \delta 142.0,139.2,135.4,134.9,133.4,131.5,128.9,128.6,128.5,128.1,127.5,127.4,127.0,126.2$, $117.4,115.5,0.5$

HRMS (EI, m/z) calcd for $\mathrm{C}_{25} \mathrm{H}_{25} \mathrm{NSi}[\mathrm{M}]^{+}$367.1756, found 367.1752 


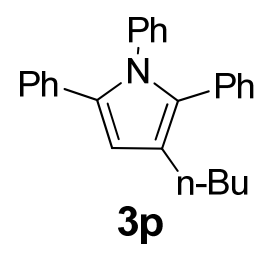

\section{3-butyl-1,2,5-triphenyl-1H-pyrrole}

White solid (93\% yield), m.p. $141-142{ }^{\circ} \mathrm{C}$

${ }^{1} \mathrm{H}$ NMR (400 MHz, $\left.\mathrm{CDCl}_{3}\right) \delta 7.21-6.99(\mathrm{~m}, 13 \mathrm{H}), 6.95-6.89(\mathrm{~m}, 2 \mathrm{H}), 6.40(\mathrm{~s}, 1 \mathrm{H}), 2.57-2.43(\mathrm{~m}, 2 \mathrm{H}), 1.67-$ $1.55(\mathrm{~m}, 2 \mathrm{H}), 1.37(\mathrm{dd}, J=14.8,7.4 \mathrm{~Hz}, 2 \mathrm{H}), 0.89(\mathrm{t}, J=7.3 \mathrm{~Hz}, 3 \mathrm{H}) .{ }^{13} \mathrm{C}$ NMR $\left(100 \mathrm{MHz}, \mathrm{CDCl}_{3}\right) \delta 139.4,134.1$, 133.6, 133.1, 132.7, 131.1, 129.0, 128.6, 128.0, 127.8, 126.8, 126.6, 126.1, 123.7, 110.5, 33.8, 26.2, 22.9, 14.2. HRMS (EI, m/z) calcd for $\mathrm{C}_{26} \mathrm{H}_{25} \mathrm{~N}[\mathrm{M}]^{+} 351.1987$, found 351.1984 


\section{Synthesis of tetrasubstituted pyrroles from propargylamines and imines}

\subsection{General procedures for the synthesis of propargylamines}

A mixture of the aldehyde $(10 \mathrm{mmol})$ and aniline $(11 \mathrm{mmol})$ was heated at $60{ }^{\circ} \mathrm{C}$ for two hours. Then, ruthenium trichloride $(10 \mathrm{~mol} \%)$, copper(I) bromide $(30 \mathrm{~mol} \%)$, phenylacetylene $(11 \mathrm{mmol})$ and water $(20 \mathrm{~mL})$ were added into the mixture under nitrogen. And the resulting mixture was stirred at $40{ }^{\circ} \mathrm{C}$ for $6-7 \mathrm{~h}$. The reaction mixture was poured into water, and extracted with diethyl ether. The organic layer was washed with water and dried over anhydrous $\mathrm{MgSO}_{4}$. The solvent was removed under reduced pressure. The product was isolated by flash column chromatography on silica gel eluting with petroleum ether/EtOAc (50/1).<smiles>C(#Cc1ccccc1)c1ccccc1</smiles>

\section{$4 a$}

N-(1,3-diphenylprop-2-yn-1-yl)aniline, known compound ${ }^{3}$<smiles>Cc1cccc(C(C#Cc2ccccc2)Nc2ccccc2)c1</smiles>

4b

\section{N-(3-phenyl-1-(m-tolyl)prop-2-yn-1-yl)aniline}

Light yellow solid, m.p. $89-90{ }^{\circ} \mathrm{C}$

${ }^{1} \mathrm{H}$ NMR (400 MHz, $\left.\mathrm{CDCl}_{3}\right) \delta 7.53-7.39(\mathrm{~m}, 4 \mathrm{H}), 7.36-7.11(\mathrm{~m}, 7 \mathrm{H}), 6.80(\mathrm{~d}, J=8.2 \mathrm{~Hz}, 3 \mathrm{H}), 5.47(\mathrm{~s}, 1 \mathrm{H}), 4.16$ (s, $1 \mathrm{H}), 2.40(\mathrm{~s}, 3 \mathrm{H}) .{ }^{13} \mathrm{C} \mathrm{NMR}\left(100 \mathrm{MHz}, \mathrm{CDCl}_{3}\right) \delta 146.8,139.8,138.7,131.9,129.3,129.0,128.9,128.4,128.2$, 124.5, 123.0, 118.7, 114.2, 88.8, 85.1, 50.8, 21.7.

HRMS calcd for $\mathrm{C}_{22} \mathrm{H}_{20} \mathrm{~N}[\mathrm{M}+\mathrm{H}]^{+} 298.1596$, found 298.1592<smiles>Cc1ccc(C(C#Cc2ccccc2)Nc2ccccc2)cc1</smiles>

4c

\section{N-(3-phenyl-1-(p-tolyl)prop-2-yn-1-yl)aniline}

Light yellow solid, m.p. $99-100{ }^{\circ} \mathrm{C}$

${ }^{1} \mathrm{H}$ NMR $\left(400 \mathrm{MHz}, \mathrm{CDCl}_{3}\right) \delta 7.53(\mathrm{~d}, J=7.9 \mathrm{~Hz}, 2 \mathrm{H}), 7.40(\mathrm{dd}, J=6.8,2.7 \mathrm{~Hz}, 2 \mathrm{H}), 7.32-7.12(\mathrm{~m}, 7 \mathrm{H}), 6.78$ (dd, $J=7.2,5.3 \mathrm{~Hz}, 3 \mathrm{H}), 5.45$ (s, 1H), $4.13(\mathrm{~s}, 1 \mathrm{H}), 2.36$ (s, 3H). ${ }^{13} \mathrm{C} \mathrm{NMR}\left(100 \mathrm{MHz}, \mathrm{CDCl}_{3}\right) \delta 146.5,137.7$, 136.7, 131.6, 129.3, 129.0, 128.1, 127.1, 122.7, 118.4, 113.9, 88.6, 84.7, 50.3, 21.0.

HRMS calcd for $\mathrm{C}_{22} \mathrm{H}_{20} \mathrm{~N}[\mathrm{M}+\mathrm{H}]^{+} 298.1596$, found 298.1598 
<smiles>C(#Cc1ccco1)c1ccccc1</smiles>

4d

\section{N-(1-(furan-2-yl)-3-phenylprop-2-yn-1-yl)aniline}

Light yellow oil

${ }^{1} \mathrm{H}$ NMR $\left(400 \mathrm{MHz}, \mathrm{CDCl}_{3}\right) \delta 7.46-7.36(\mathrm{~m}, 3 \mathrm{H}), 7.30-7.16(\mathrm{~m}, 5 \mathrm{H}), 6.81(\mathrm{dd}, J=4.7,3.7 \mathrm{~Hz}, 3 \mathrm{H}), 6.50(\mathrm{~d}, J=$ $3.1 \mathrm{~Hz}, 1 \mathrm{H}), 6.37(\mathrm{dd}, J=2.9,1.7 \mathrm{~Hz}, 1 \mathrm{H}), 5.57(\mathrm{~s}, 1 \mathrm{H}), 4.21(\mathrm{~s}, 1 \mathrm{H}) .{ }^{13} \mathrm{C} \mathrm{NMR}\left(100 \mathrm{MHz}, \mathrm{CDCl}_{3}\right) \delta 152.1,146.2$, $142.9,132.0,129.3,128.6,128.4,122.6,119.3,114.7,110.6,107.8,86.3,84.2,45.2$.

HRMS calcd for $\mathrm{C}_{19} \mathrm{H}_{16} \mathrm{NO}[\mathrm{M}+\mathrm{H}]^{+} 274.1232$, found 274.1236<smiles>Cc1cccc(NC(C#Cc2ccccc2)c2ccccc2)c1</smiles>

\section{N-(1,3-diphenylprop-2-yn-1-yl)-3-methylaniline}

Light yellow oil

${ }^{1} \mathrm{H}$ NMR $\left(400 \mathrm{MHz}, \mathrm{CDCl}_{3}\right) \delta 7.67(\mathrm{~d}, J=7.6 \mathrm{~Hz}, 2 \mathrm{H}), 7.45-7.26(\mathrm{~m}, 8 \mathrm{H}), 7.12(\mathrm{t}, J=7.8 \mathrm{~Hz}, 1 \mathrm{H}), 6.75-6.40$ $(\mathrm{m}, 3 \mathrm{H}), 5.51(\mathrm{~s}, 1 \mathrm{H}), 4.12(\mathrm{~s}, 1 \mathrm{H}), 2.31(\mathrm{~s}, 3 \mathrm{H}) .{ }^{13} \mathrm{C} \mathrm{NMR}\left(100 \mathrm{MHz}, \mathrm{CDCl}_{3}\right) \delta 146.5,139.8,138.8,131.6,128.9$, $128.6,128.2,128.1,127.9,127.2,122.7,119.4,114.8,111.0,88.5,84.8,50.5,21.5$.

HRMS calcd for $\mathrm{C}_{22} \mathrm{H}_{20} \mathrm{~N}[\mathrm{M}+\mathrm{H}]^{+} 298.1596$, found 298.1591 


\subsection{Screen reaction conditions}<smiles>FC(C#Cc1ccccc1)Nc1ccccc1</smiles>

2a

$4 a$<smiles>c1ccc(-c2cc(-c3ccccc3)n(-c3ccccc3)c2-c2ccccc2)cc1</smiles>

3a

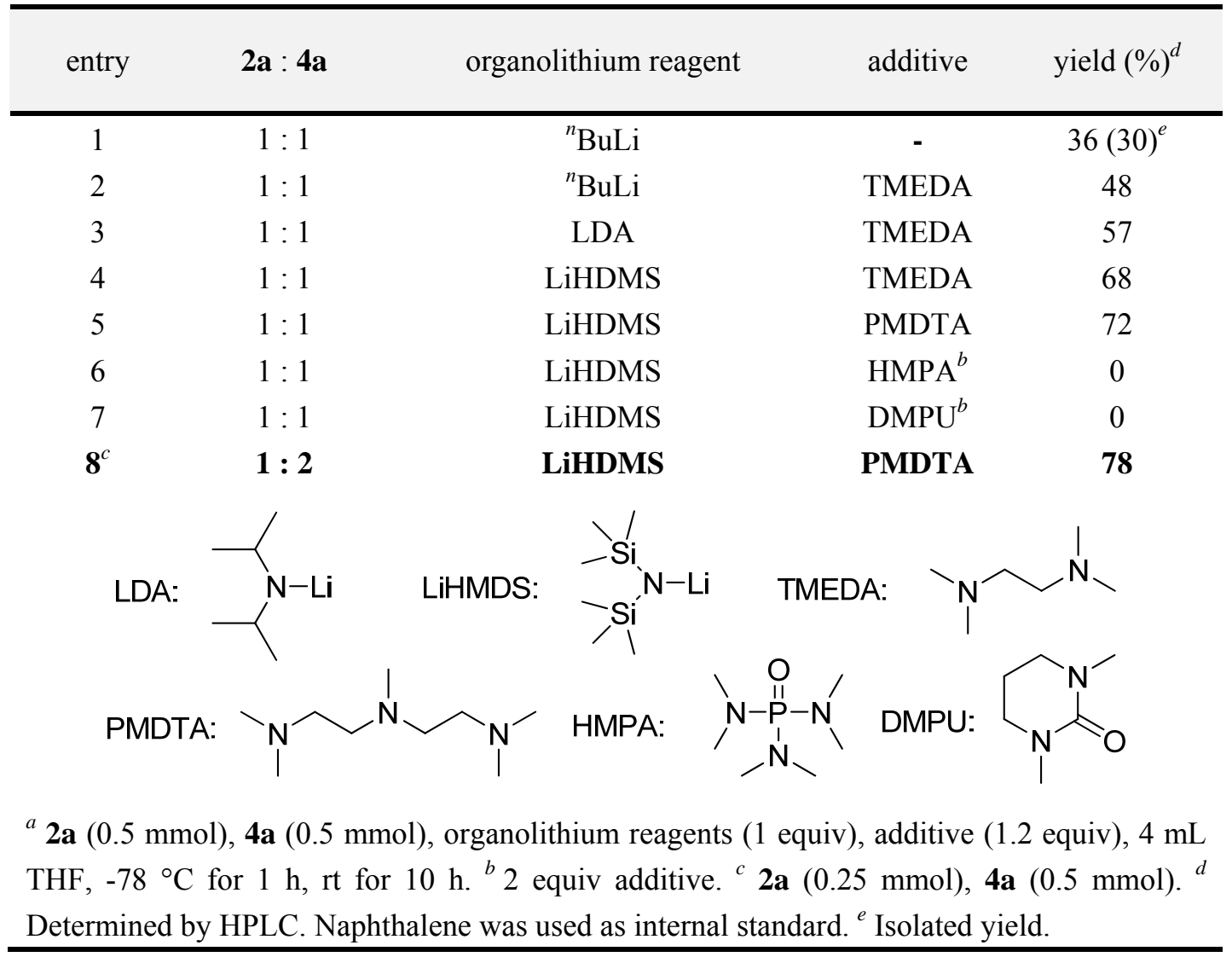

\subsection{Typical procedures for the synthesis of pyrroles from propargylamines and imines}

To a solution of propargylamine $4(0.5 \mathrm{mmol})$ in freshly distilled THF $(2 \mathrm{~mL})$ was added $N, N, N^{\prime}, N^{\prime \prime}, N^{\prime \prime}$-pentamethyldiethylenetriamine (PMDTA, 1.2 equiv). The resulting mixture was cooled to $-78^{\circ} \mathrm{C}$ and treated with LiHMDS ( $1 \mathrm{M}$ in THF, 1 equiv) dropwise. The resulting dark red solution was stirred at $-78{ }^{\circ} \mathrm{C}$ for 1 hour, treated with a solution of imine $2(0.25 \mathrm{mmol})$ in THF $(2 \mathrm{~mL})$ dropwise, stirred for $10 \mathrm{~h}$ at room temperature and quenched with water. The aqueous layer was extracted with $\mathrm{Et}_{2} \mathrm{O}$ and the combined organic layers were washed twice with brine, dried over $\mathrm{MgSO}_{4}$, filtered and concentrated under reduced pressure. The crude residue was purified by flash chromatography over silica gel (petroleum ether/EtOAc: 100/1) or direct crystallization from $\mathrm{EtOH} /$ pentane to yield the desired pyrrole 3 . 
<smiles>COc1ccccc1-c1[nH]c(-c2ccccc2)cc1-c1ccccc1</smiles>

2-(2-methoxyphenyl)-1,3,5-triphenyl-1H-pyrrole, $86 \%$ yield White solid, m.p. $162-163{ }^{\circ} \mathrm{C}$

${ }^{1} \mathrm{H}$ NMR (400 MHz, $\left.\mathrm{CDCl}_{3}\right) \delta 7.30-7.06(\mathrm{~m}, 15 \mathrm{H}), 6.98(\mathrm{~m}, 2 \mathrm{H}), 6.83-6.75(\mathrm{~m}, 2 \mathrm{H}), 6.68(\mathrm{~d}, J=8.2 \mathrm{~Hz}, 1 \mathrm{H})$, 3.36 (s, 3H). ${ }^{13} \mathrm{C}$ NMR $\left(100 \mathrm{MHz}, \mathrm{CDCl}_{3}\right) \delta 158.3$, 139.4, 136.4, 134.7, 133.6, 133.3, 129.7, 128.5, 128.2, 128.1, $128.0,127.5,126.8,126.3,125.4,123.7,122.3,120.5,110.8,109.7,55.0$.

HRMS calcd for $\mathrm{C}_{29} \mathrm{H}_{23} \mathrm{NONa}[\mathrm{M}+\mathrm{Na}]^{+} 424.1677$, found 424.1686<smiles>Cc1cccc(-c2c(Br)cc(-c3ccccc3)n2-c2ccccc2)c1</smiles>

1,3,5-triphenyl-2-(m-tolyl)-1H-pyrrole, $83 \%$ yield

White solid, m.p. $154-155{ }^{\circ} \mathrm{C}$

${ }^{1} \mathrm{H}$ NMR $\left(400 \mathrm{MHz}, \mathrm{CDCl}_{3}\right) \delta 7.30-7.09(\mathrm{~m}, 13 \mathrm{H}), 7.05-6.91(\mathrm{~m}, 4 \mathrm{H}), 6.87-6.80(\mathrm{~m}, 2 \mathrm{H}), 6.71(\mathrm{~s}, 1 \mathrm{H}), 2.13(\mathrm{~s}$, $3 \mathrm{H}) .{ }^{13} \mathrm{C}$ NMR $\left(100 \mathrm{MHz}, \mathrm{CDCl}_{3}\right) \delta 139.0,137.4,136.3,134.8,133.1,132.7,132.3,131.6,129.2,128.7,128.5$, $128.2,128.1,127.8,127.2,126.4,125.5,123.4,110.0,21.4$.

HRMS (EI, m/z) calcd for $\mathrm{C}_{29} \mathrm{H}_{23} \mathrm{~N}[\mathrm{M}]^{+} 385.1830$, found 385.1834<smiles>Brc1cccc(-c2c(-c3ccccc3)cc(-c3ccccc3)n2-c2ccccc2)c1</smiles>

3s

2-(3-bromophenyl)-1,3,5-triphenyl-1H-pyrrole, 79\% yield

Light yellow solid, m.p. $179-180^{\circ} \mathrm{C}$

${ }^{1} \mathrm{H}$ NMR $\left(400 \mathrm{MHz}, \mathrm{CDCl}_{3}\right) \delta 7.28-7.08(\mathrm{~m}, 15 \mathrm{H}), 7.01-6.92(\mathrm{~m}, 4 \mathrm{H}), 6.68(\mathrm{~s}, 1 \mathrm{H}) .{ }^{13} \mathrm{C} \mathrm{NMR}(100 \mathrm{MHz}$, $\left.\mathrm{CDCl}_{3}\right) \delta 138.6,135.8,135.5,134.9,134.3,132.8,130.5,130.2,130.1,129.4,129.2,128.8,128.7,128.4,128.1$, 127.6, 126.7, 126.0, 124.3, 121.8, 110.3.

HRMS (EI, m/z) calcd for $\mathrm{C}_{28} \mathrm{H}_{20} \mathrm{BrN}[\mathrm{M}]^{+} 449.0779$, found 449.0787 
<smiles>Cc1ccc(-c2[nH]c(-c3ccccc3)cc2-c2ccccc2)cc1</smiles>

$3 t$

1,3,5-triphenyl-2-(p-tolyl)-1H-pyrrole, $86 \%$ yield

White solid, m.p. $160-161{ }^{\circ} \mathrm{C}$

${ }^{1} \mathrm{H}$ NMR $\left(400 \mathrm{MHz}, \mathrm{CDCl}_{3}\right) \delta 7.27-7.07(\mathrm{~m}, 13 \mathrm{H}), 7.01-6.83(\mathrm{~m}, 6 \mathrm{H}), 6.70(\mathrm{~s}, 1 \mathrm{H}), 2.25(\mathrm{~s}, 3 \mathrm{H}) .{ }^{13} \mathrm{C}$ NMR $(100$ $\left.\mathrm{MHz}, \mathrm{CDCl}_{3}\right) \delta 139.1,136.7,136.4,134.8,133.1,132.4,131.4,129.8,129.3,128.8,128.7,128.6,128.3,128.1$, 127.2, 126.4, 125.6, 123.4, 110.1, 21.4.

HRMS (EI, $\mathrm{m} / \mathrm{z}$ ) calcd for $\mathrm{C}_{29} \mathrm{H}_{23} \mathrm{~N}[\mathrm{M}]^{+} 385.1830$, found 385.1837<smiles>FC(F)(F)c1ccc(-c2c(-c3ccccc3)cc(-c3ccccc3)n2-c2ccccc2)cc1</smiles>

$3 \mathbf{u}$

1,3,5-triphenyl-2-(4-(trifluoromethyl)phenyl)-1H-pyrrole, $66 \%$ yield

White solid, m.p. $162-163{ }^{\circ} \mathrm{C}$

${ }^{1} \mathrm{H}$ NMR $\left(500 \mathrm{MHz}, \mathrm{CDCl}_{3}\right) \delta 7.36(\mathrm{~d}, J=8.1 \mathrm{~Hz}, 2 \mathrm{H}), 7.25-7.16(\mathrm{~m}, 11 \mathrm{H}), 7.11(\mathrm{dd}, J=7.7,1.9 \mathrm{~Hz}, 4 \mathrm{H}), 7.00$ $6.94(\mathrm{~m}, 2 \mathrm{H}), 6.68(\mathrm{~s}, 1 \mathrm{H}) .{ }^{13} \mathrm{C} \mathrm{NMR}\left(126 \mathrm{MHz}, \mathrm{CDCl}_{3}\right) \delta 138.7,136.5,135.9(\mathrm{~d}, J=6.1 \mathrm{~Hz}), 132.8,131.6,130.4$, 129.2, 128.9, 128.7, 128.5, 128.2, 127.7, 126.8, 126.1, 125.4, 124.9 (d, $J=3.8 \mathrm{~Hz}), 124.4$ (q, $J=272.2 \mathrm{~Hz}), 110.7$. ${ }^{19} \mathrm{~F}$ NMR $\left(471 \mathrm{MHz}, \mathrm{CDCl}_{3}\right) \delta-62.5$.

HRMS (EI, m/z) calcd for $\mathrm{C}_{29} \mathrm{H}_{20} \mathrm{~F}_{3} \mathrm{~N}[\mathrm{M}]^{+} 439.1548$, found 439.1548<smiles>Cc1ccc(-c2c(-c3ccccc3)cc(-c3ccccc3)n2-c2ccccc2)cc1</smiles>

3v

3,5-diphenyl-1-(m-tolyl)-2-(p-tolyl)-1H-pyrrole, 73\% yield White solid, m.p. $146-147^{\circ} \mathrm{C}$

${ }^{1} \mathrm{H}$ NMR $\left(500 \mathrm{MHz}, \mathrm{CDCl}_{3}\right) \delta 7.27-7.11(\mathrm{~m}, 10 \mathrm{H}), 7.06-6.90(\mathrm{~m}, 6 \mathrm{H}), 6.82-6.75(\mathrm{~m}, 2 \mathrm{H}), 6.69(\mathrm{~s}, 1 \mathrm{H}), 2.26(\mathrm{~s}$, $3 \mathrm{H}), 2.15(\mathrm{~s}, 3 \mathrm{H}) .{ }^{13} \mathrm{C}$ NMR $\left(126 \mathrm{MHz}, \mathrm{CDCl}_{3}\right) \delta 138.8,138.3,136.5,136.4,134.7,133.1,132.4,131.3,129.8$, 128.6, 128.5, 128.2, 128.1, 127.9, 127.8, 126.2, 125.4, 123.2, 109.9, 21.3, 21.2.

HRMS calcd for $\mathrm{C}_{30} \mathrm{H}_{26} \mathrm{~N}[\mathrm{M}+\mathrm{H}]^{+} 400.2065$, found 400.2059 


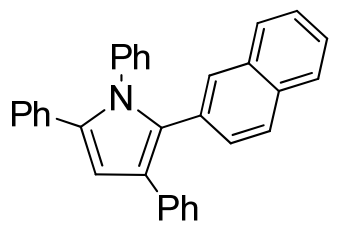

$3 w$

2-(naphthalen-2-yl)-1,3,5-triphenyl-1H-pyrrole, $86 \%$ yield

White solid, m.p. $209-210{ }^{\circ} \mathrm{C}$

${ }^{1} \mathrm{H}$ NMR $\left(400 \mathrm{MHz}, \mathrm{CDCl}_{3}\right) \delta 7.73(\mathrm{~d}, J=7.8 \mathrm{~Hz}, 1 \mathrm{H}), 7.57(\mathrm{dd}, J=16.0,8.2 \mathrm{~Hz}, 2 \mathrm{H}), 7.49(\mathrm{~s}, 1 \mathrm{H}), 7.43-7.32$ $(\mathrm{m}, 2 \mathrm{H}), 7.27(\mathrm{~d}, J=7.2 \mathrm{~Hz}, 2 \mathrm{H}), 7.22-7.08(\mathrm{~m}, 12 \mathrm{H}), 7.06-6.94(\mathrm{~m}, 2 \mathrm{H}), 6.75(\mathrm{~s}, 1 \mathrm{H}) .{ }^{13} \mathrm{C} \mathrm{NMR}(100 \mathrm{MHz}$, $\left.\mathrm{CDCl}_{3}\right) \delta 138.9,136.2,135.2,133.2,133.0,132.3,131.6,130.8,130.3,129.4,129.3,128.8,128.7,128.4,128.3$, $128.2,128.1,127.7,127.4,127.3,126.5,126.1,125.9,125.7,124.1,110.4$.

HRMS (EI, m/z) calcd for $\mathrm{C}_{32} \mathrm{H}_{23} \mathrm{~N}[\mathrm{M}]^{+} 421.1830$, found 421.1838<smiles></smiles>

$3 \mathbf{x}$

1,2,3-triphenyl-5-(m-tolyl)-1H-pyrrole, $86 \%$ yield

Light yellow solid, m.p. $166-167^{\circ} \mathrm{C}$

${ }^{1} \mathrm{H}$ NMR $\left(400 \mathrm{MHz}, \mathrm{CDCl}_{3}\right) \delta 7.33-6.96(\mathrm{~m}, 18 \mathrm{H}), 6.87(\mathrm{~d}, J=7.6 \mathrm{~Hz}, 1 \mathrm{H}), 6.73(\mathrm{~s}, 1 \mathrm{H}), 2.24(\mathrm{~s}, 3 \mathrm{H}) .{ }^{13} \mathrm{C} \mathrm{NMR}$ $\left(100 \mathrm{MHz}, \mathrm{CDCl}_{3}\right) \delta 139.0,137.6,136.3,135.1,132.9,132.2,131.6,129.5,129.3,128.6,128.3,128.0,127.9$, $127.3,127.2,127.1,125.8,125.6,123.6,110.0,21.6$.

HRMS (EI, m/z) calcd for $\mathrm{C}_{29} \mathrm{H}_{23} \mathrm{~N}[\mathrm{M}]^{+} 385.1830$, found 385.1838<smiles>Cc1ccc(-c2cc(-c3ccccc3)c(-c3ccccc3)[nH]2)cc1</smiles>

$3 y$

1,2,3-triphenyl-5-(p-tolyl)-1H-pyrrole, $83 \%$ yield

White solid, m.p. $173-174{ }^{\circ} \mathrm{C}$

${ }^{1} \mathrm{H}$ NMR $\left(500 \mathrm{MHz}, \mathrm{CDCl}_{3}\right) \delta 7.25-7.09(\mathrm{~m}, 11 \mathrm{H}), 7.06-6.95(\mathrm{~m}, 8 \mathrm{H}), 6.66(\mathrm{~s}, 1 \mathrm{H}), 2.28(\mathrm{~s}, 3 \mathrm{H}) .{ }^{13} \mathrm{C} \mathrm{NMR}(100$ $\left.\mathrm{MHz}, \mathrm{CDCl}_{3}\right) \delta 139.1,136.4,136.2,135.1,132.9,132.1,131.6,130.2,129.3,128.9,128.6,128.6,128.3,128.2$, 128.0, 127.2, 127.0, 125.6, 123.5, 109.8, 21.3.

HRMS (EI, m/z) calcd for $\mathrm{C}_{29} \mathrm{H}_{23} \mathrm{~N}[\mathrm{M}]^{+} 385.1830$, found 385.1834 


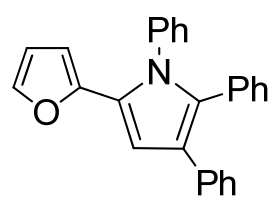

$3 z$

5-(furan-2-yl)-1,2,3-triphenyl-1H-pyrrole, 50\% yield

Light yellow solid, m.p. $107-108^{\circ} \mathrm{C}$

${ }^{1} \mathrm{H}$ NMR (400 MHz, $\left.\mathrm{CDCl}_{3}\right) \delta 7.34-7.00(\mathrm{~m}, 16 \mathrm{H}), 6.89(\mathrm{~s}, 1 \mathrm{H}), 6.18(\mathrm{dd}, J=3.4,1.8 \mathrm{~Hz}, 1 \mathrm{H}), 5.29$ (d, $J=3.3$

$\mathrm{Hz}, 1 \mathrm{H}) .{ }^{13} \mathrm{C} \mathrm{NMR}\left(100 \mathrm{MHz}, \mathrm{CDCl}_{3}\right) \delta 147.5,140.9,139.0,136.0,132.9,132.3,131.5,129.4,128.8,128.2,128.0$, $127.2,126.6,125.7,123.6,110.9,108.6,105.3$.

HRMS (EI, m/z) calcd for $\mathrm{C}_{26} \mathrm{H}_{19} \mathrm{NO}[\mathrm{M}]^{+} 361.1467$, found 361.1476

\section{References}

(1) Baricordi, N.; Benetti, S.; Biondini, G.; Risi, C. D.; Pollini, G. P. Tetrahedron Lett. 2004, 45, 1373.

(2) (a) Li, C. J.; Wei, C. M. Chem. Commun. 2002, 268. (b) Katritzky, A. R.; Li, J. Q.; Gordeev, M. F. Synthesis 1994, 93.

(3) Wei, C. M.; Mague, J. T.; Li, C. J. PNAS 2004, 101, 5749. 


\section{Copy of NMR spectroscopy}

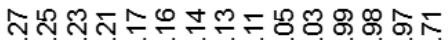

nNañNánogón

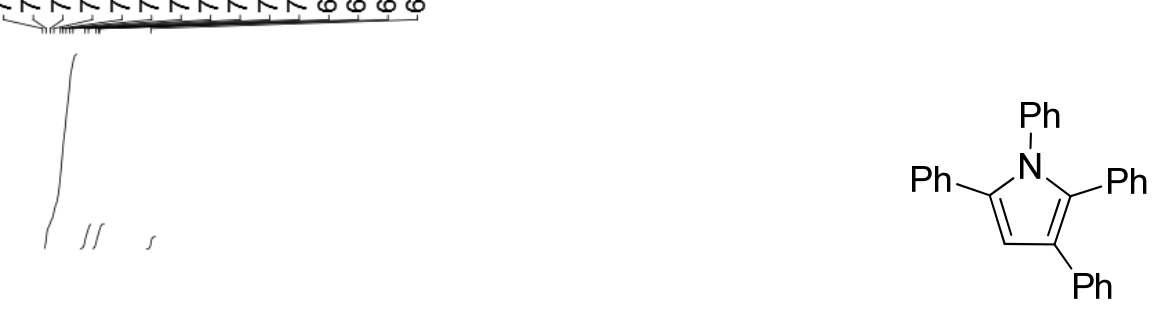

$3 a$
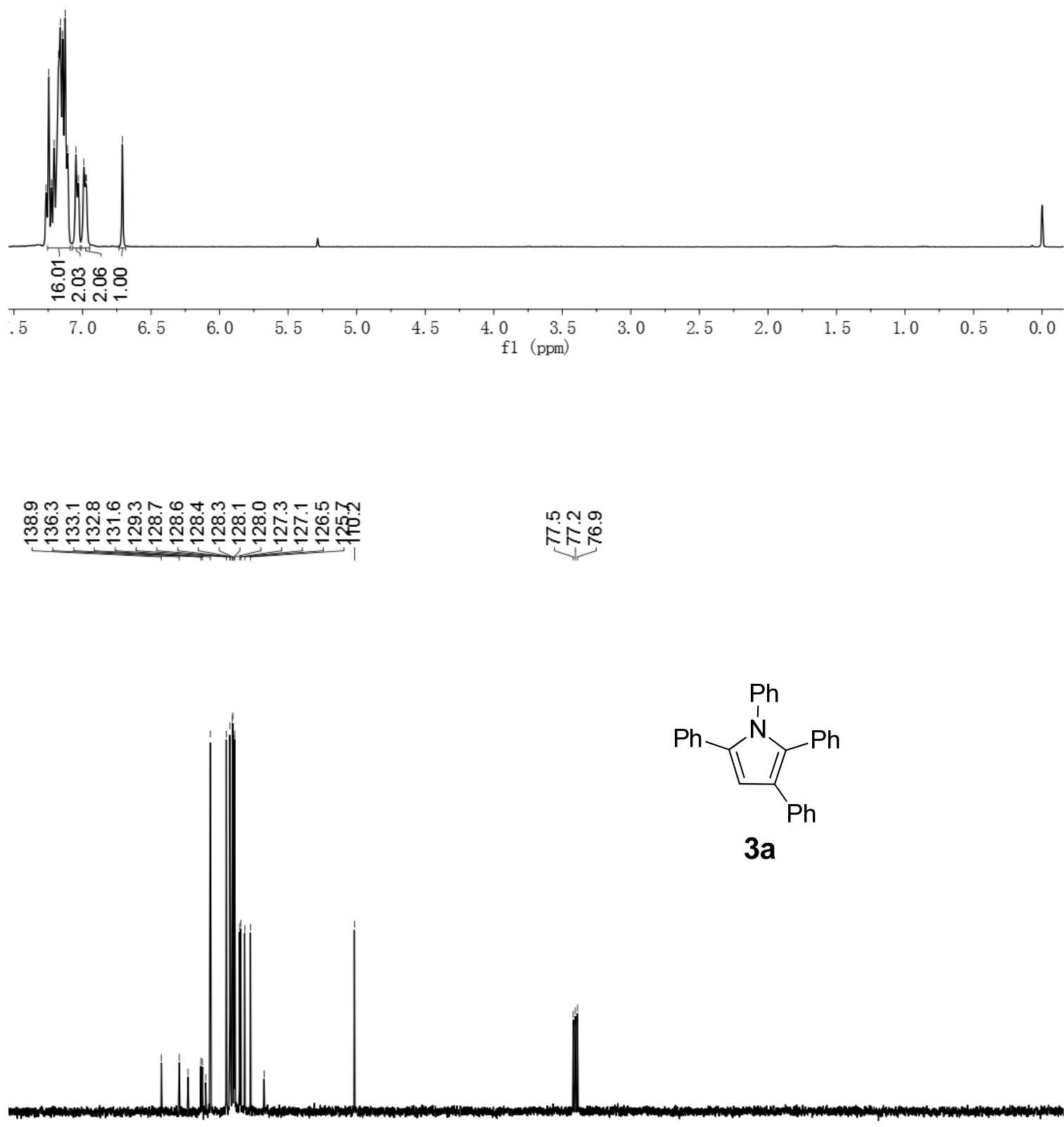

$3 a$

160

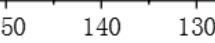

120

$110 \quad 100$

$\begin{array}{rr}90 & 80 \\ \text { f1 } & (\mathrm{ppm})\end{array}$ 
<smiles>COc1ccc(-c2cc(-c3ccccc3)c(-c3ccccc3)[nH]2)cc1</smiles>

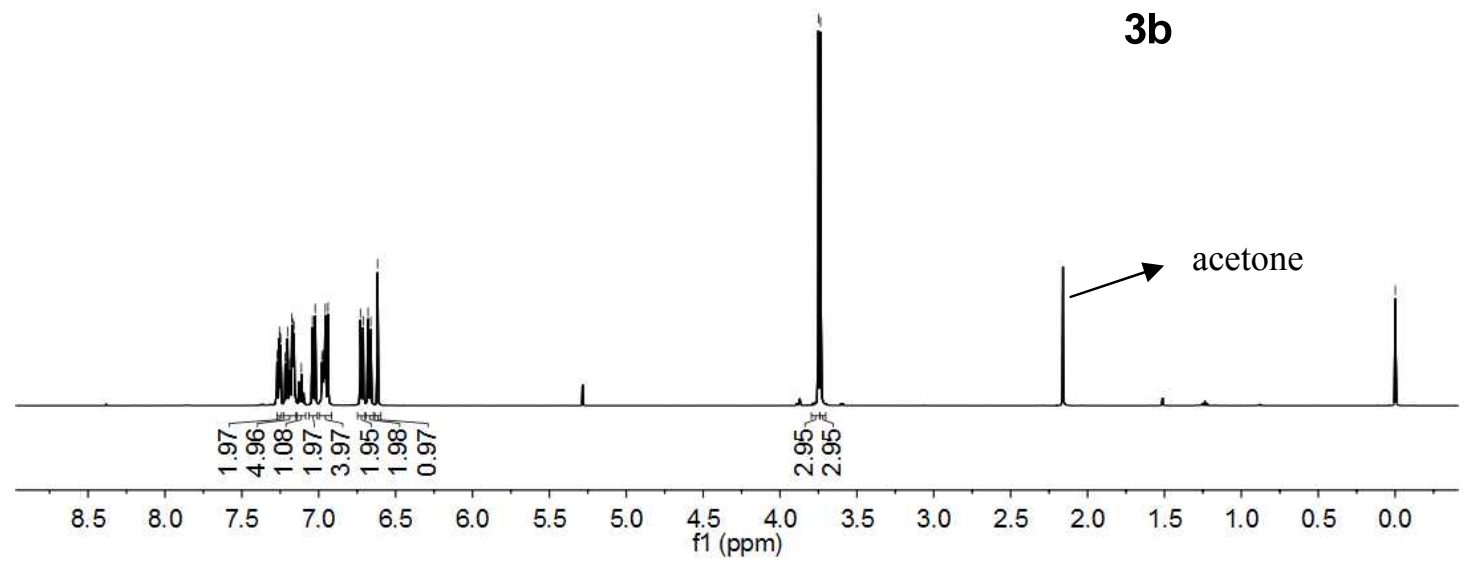<smiles>COc1ccc(-c2cc(-c3ccccc3)c(-c3ccccc3)[nH]2)cc1</smiles>

$3 b$

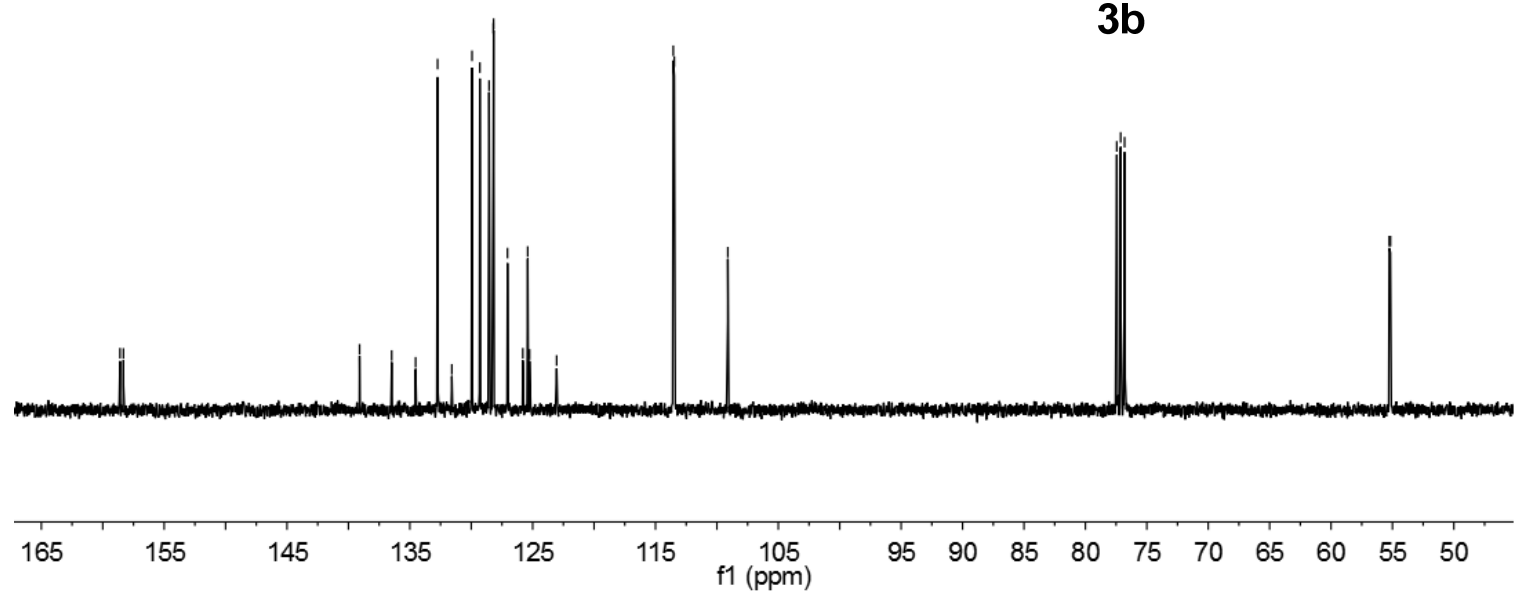



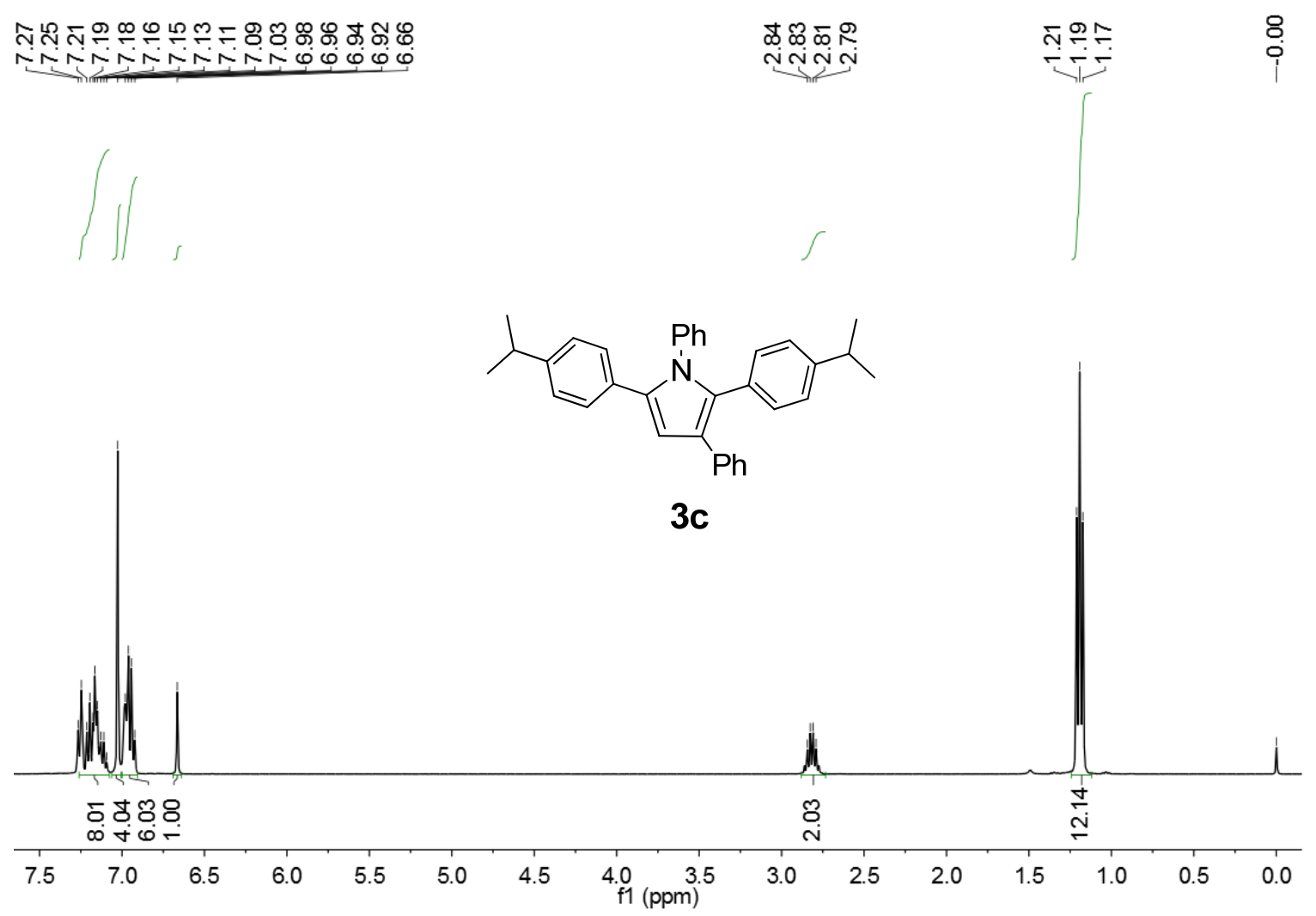

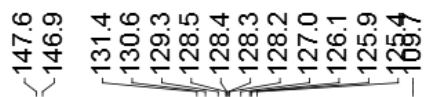

R̂́

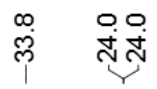<smiles>CC(C)c1ccc(-c2cc(-c3ccccc3)c(-c3ccc(C(C)C)cc3)n2P)cc1</smiles>

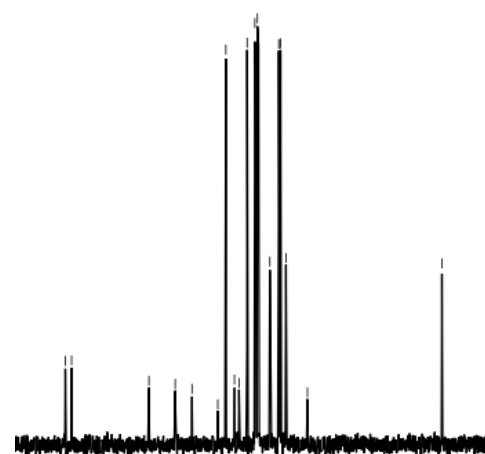

3c

\begin{tabular}{|c|c|c|c|c|c|c|c|c|c|c|c|c|c|}
\hline 145 & 135 & 125 & 115 & 105 & 95 & 85 & $\begin{array}{c}75 \\
\text { f1 (ppm) }\end{array}$ & 65 & 55 & 45 & 35 & 25 & 15 \\
\hline
\end{tabular}




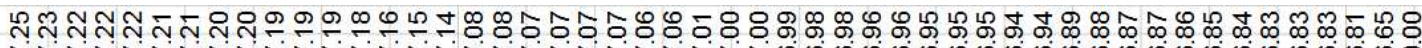

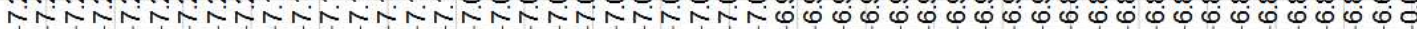

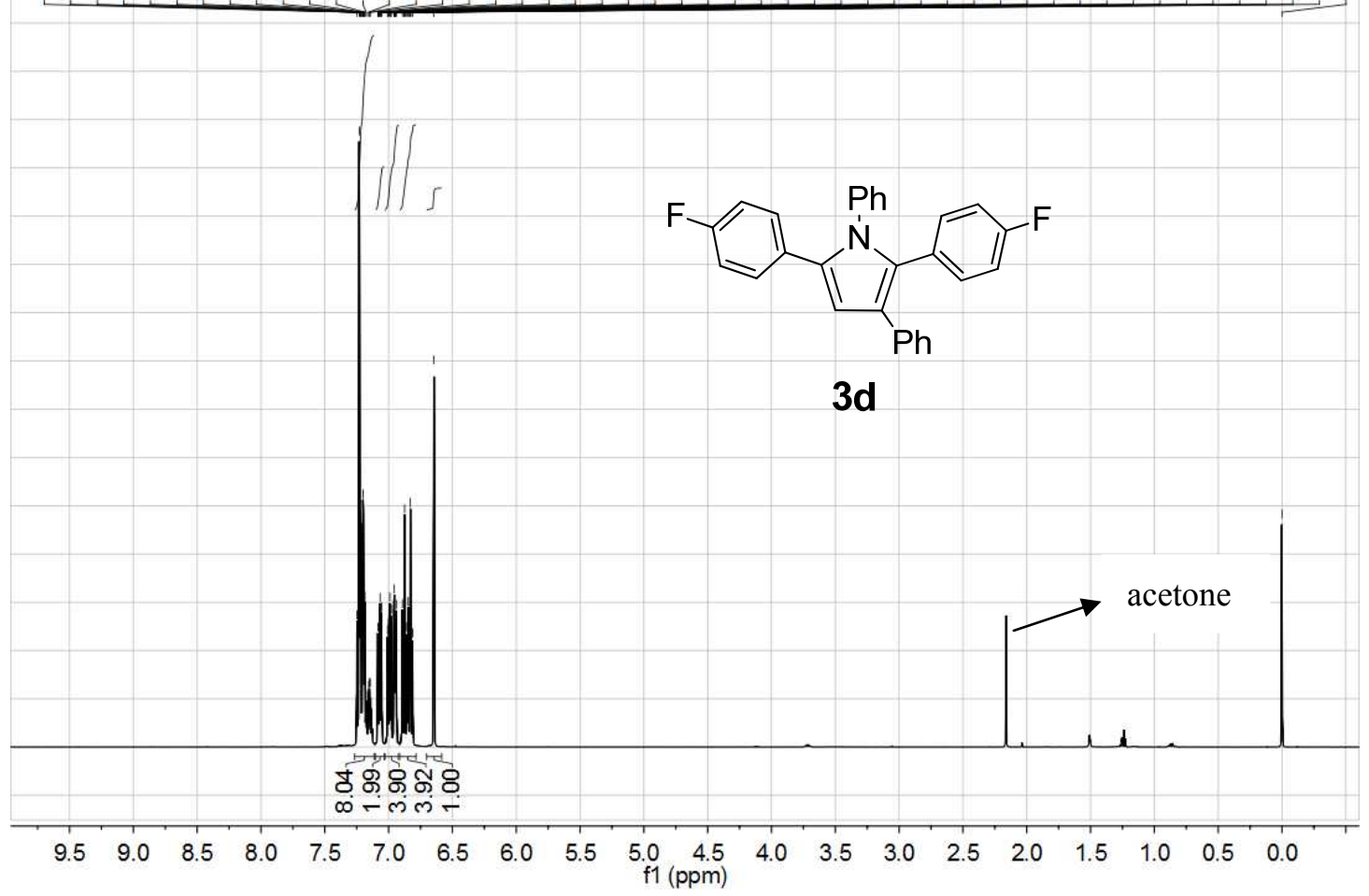

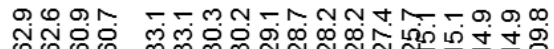

ஸู่

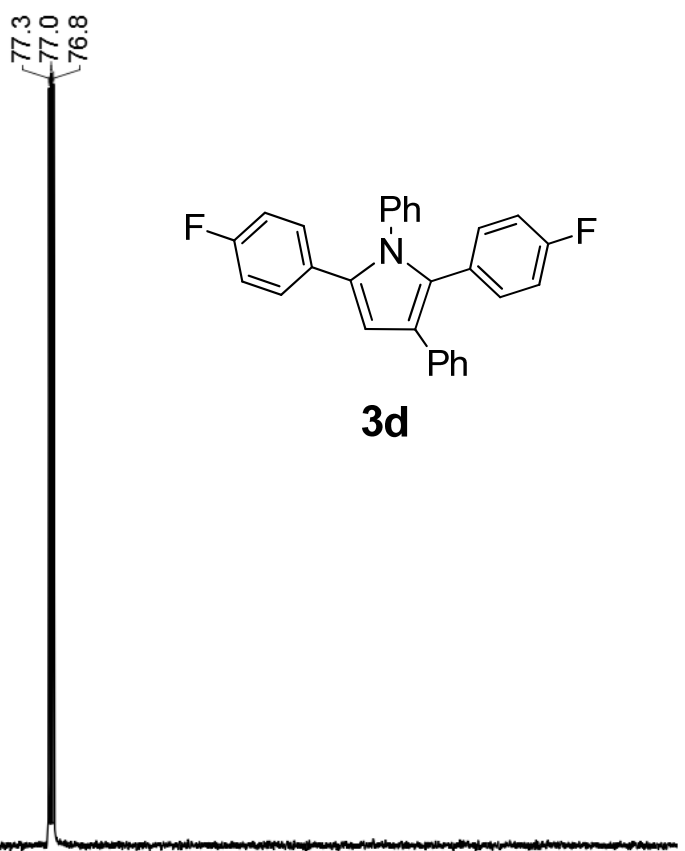

$\begin{array}{lllllllllllllll}160 & 150 & 140 & 130 & 120 & 110 & 100 & \underset{\mathrm{f} 1(\mathrm{ppm})}{90} 80 & 70 & 60 & 50 & 40 & 30 & 20 & 16\end{array}$


욛
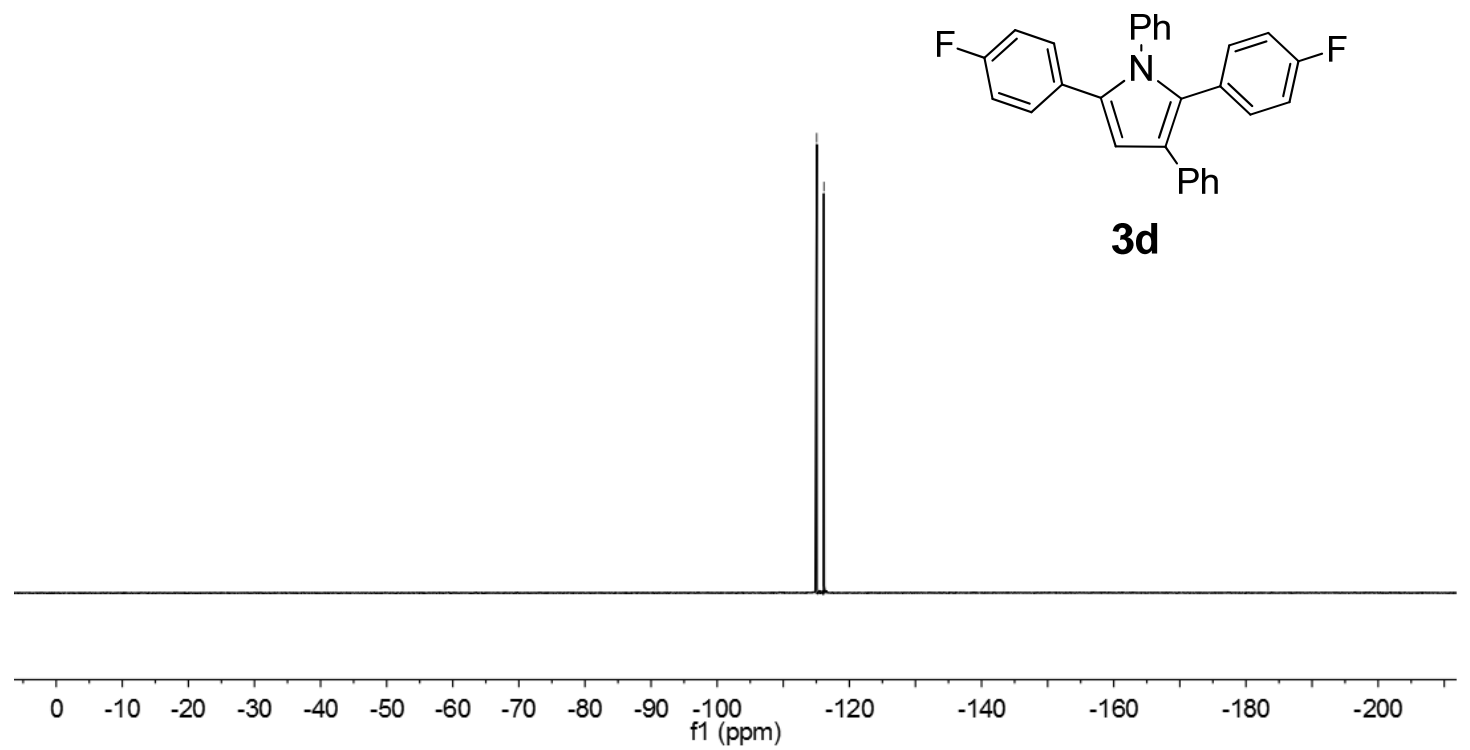


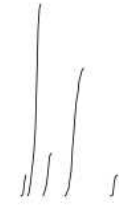<smiles>Brc1cccc(-c2cc(-c3ccccc3)c(-c3ccccc3)[nH]2)c1</smiles>

$3 e$
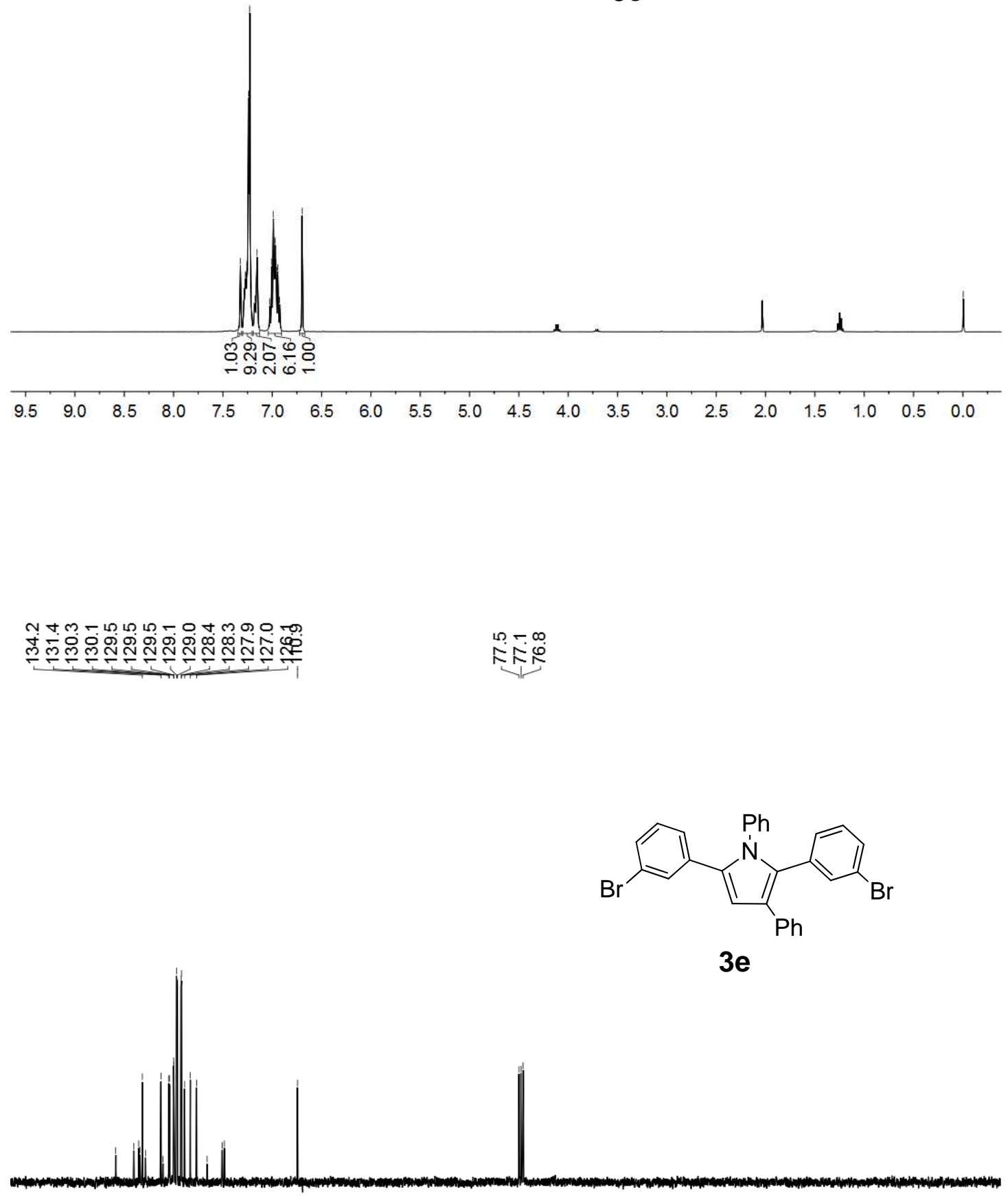


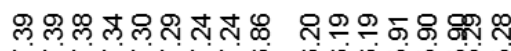

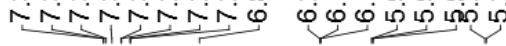
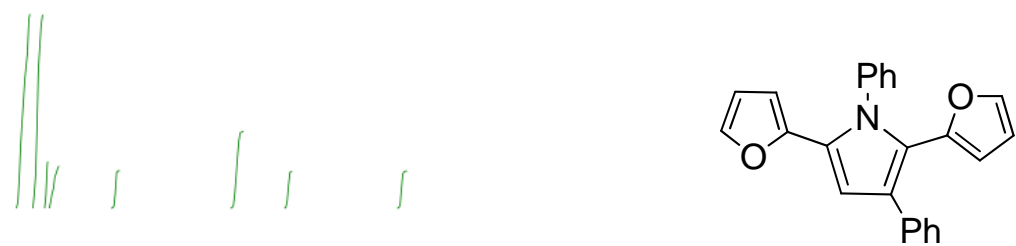

3f
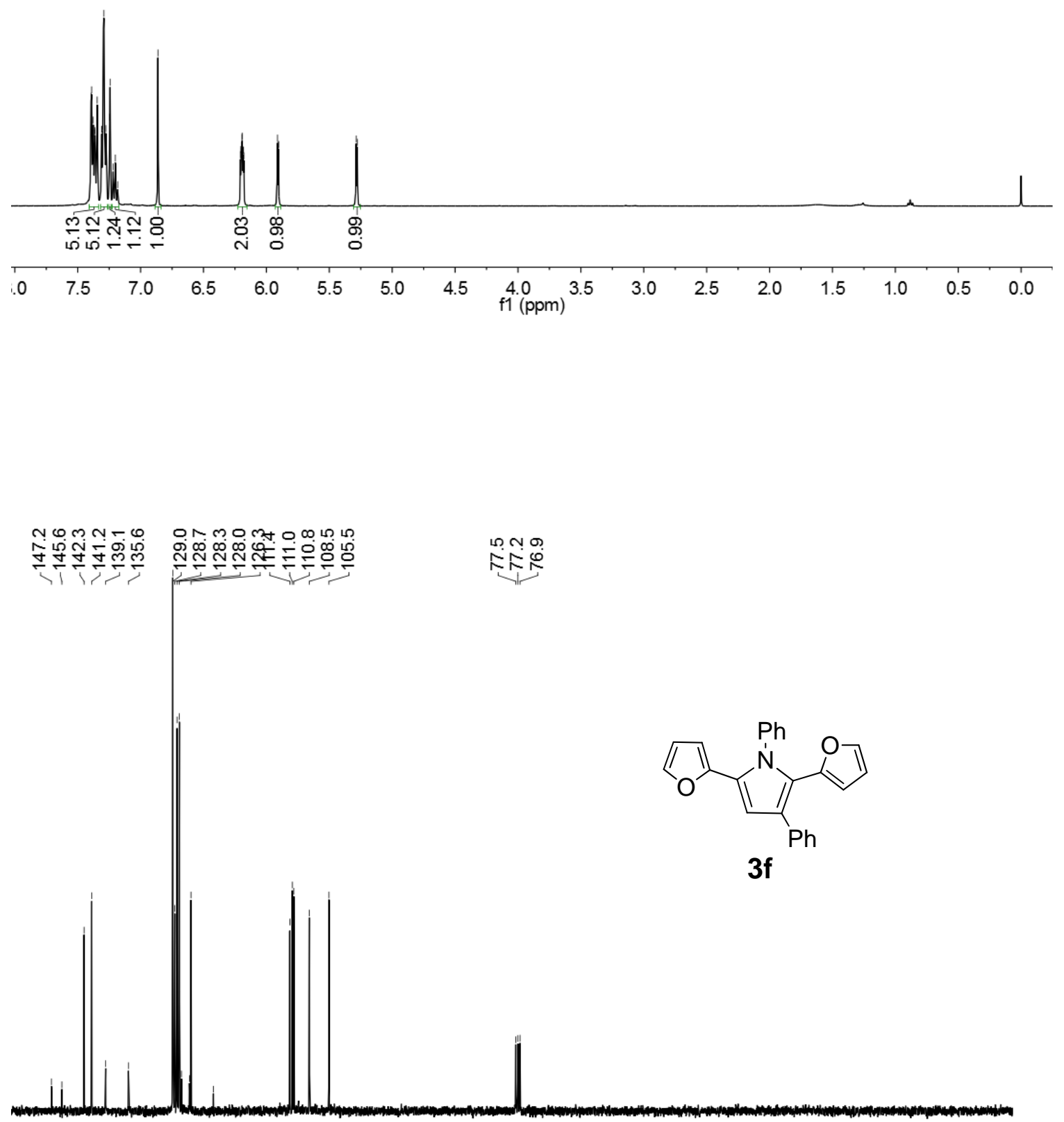

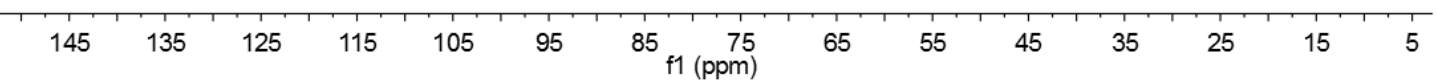




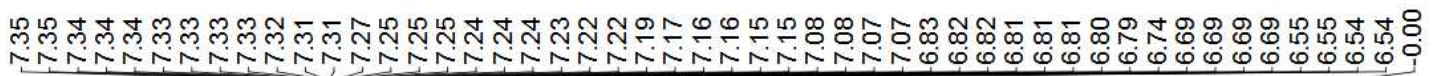

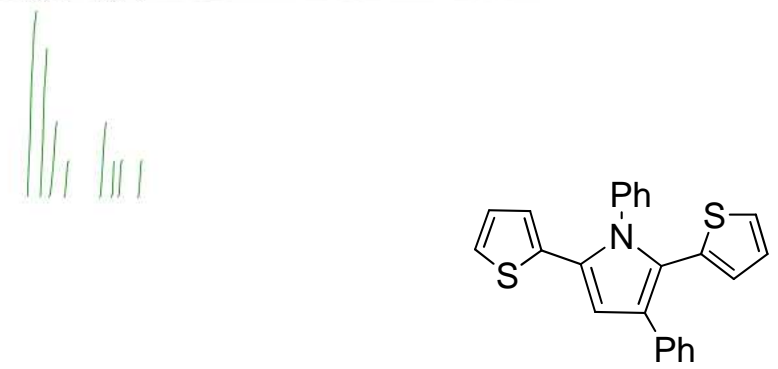

$3 g$
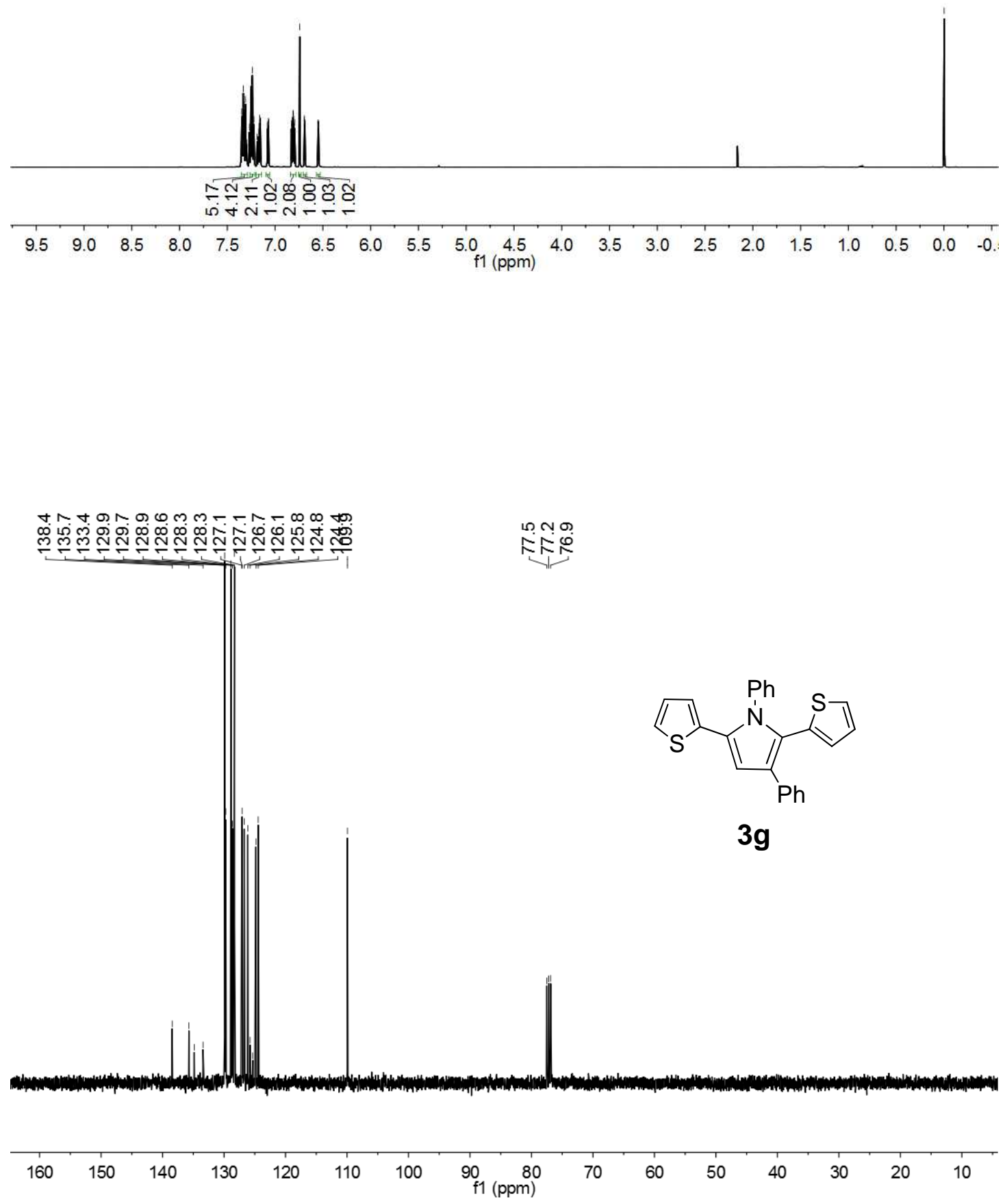


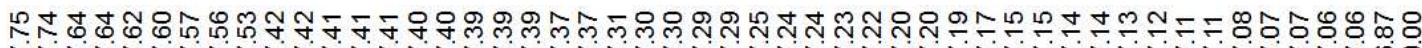

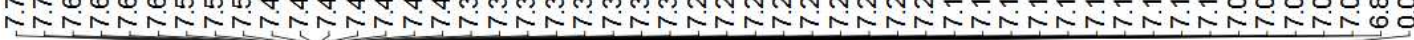
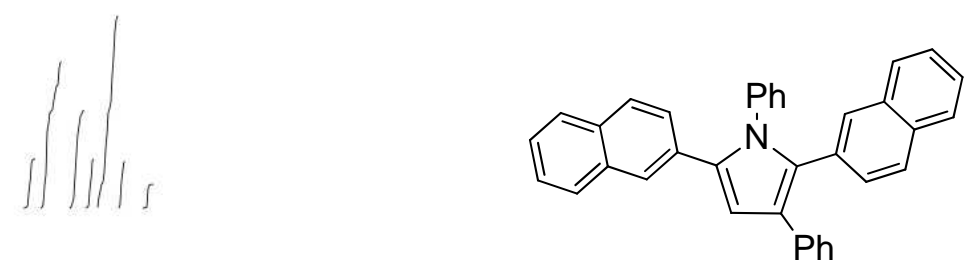

$3 h$

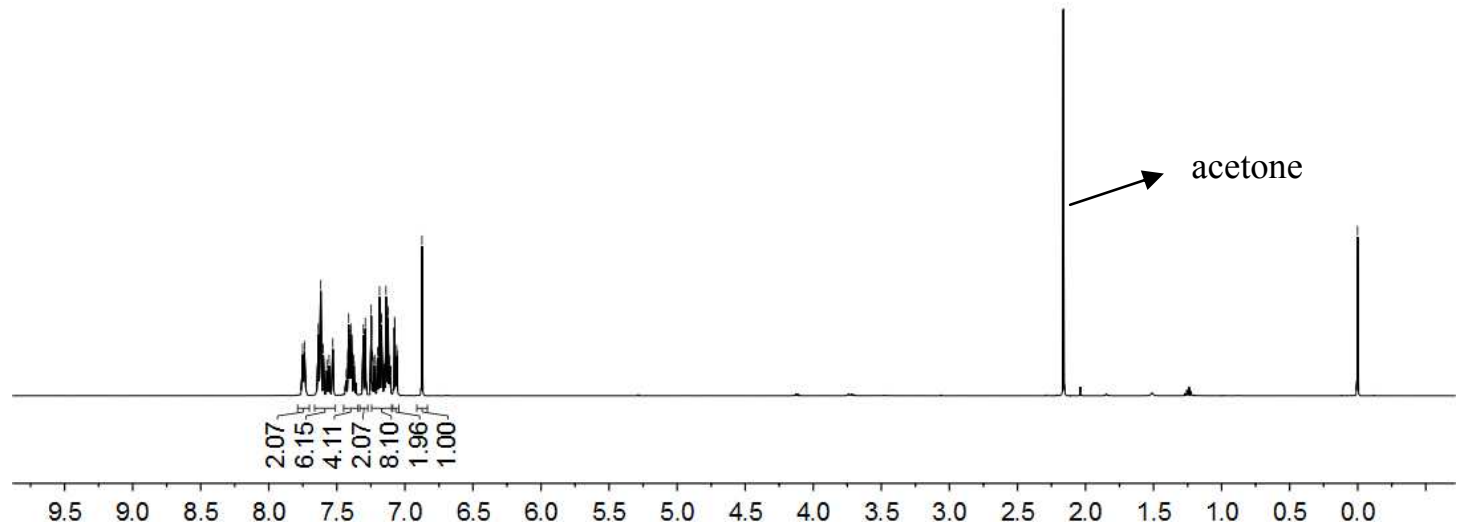

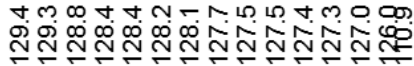

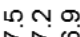

篎禺
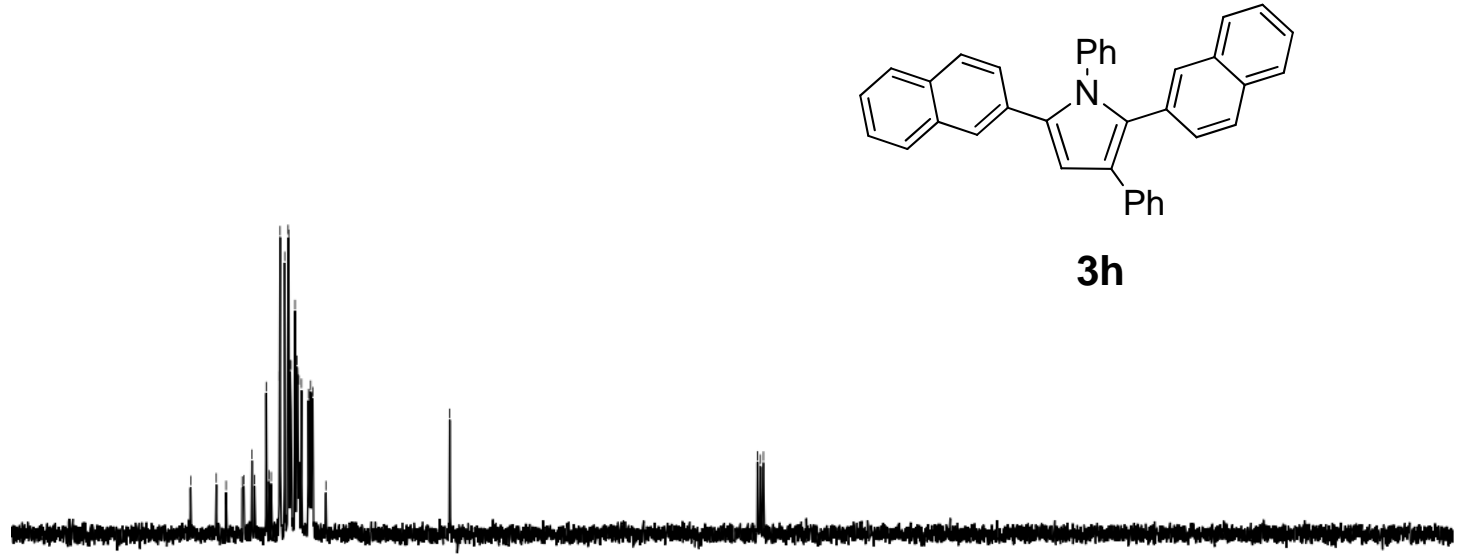

150

$140 \quad 130$

120

110

100

90

f1 $(\mathrm{ppm})$

$70 \quad 60$

50

$40 \quad 30$

$20 \quad 10$ 


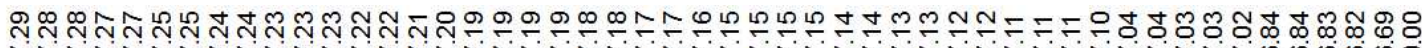

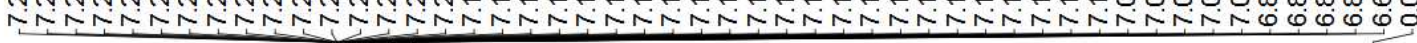
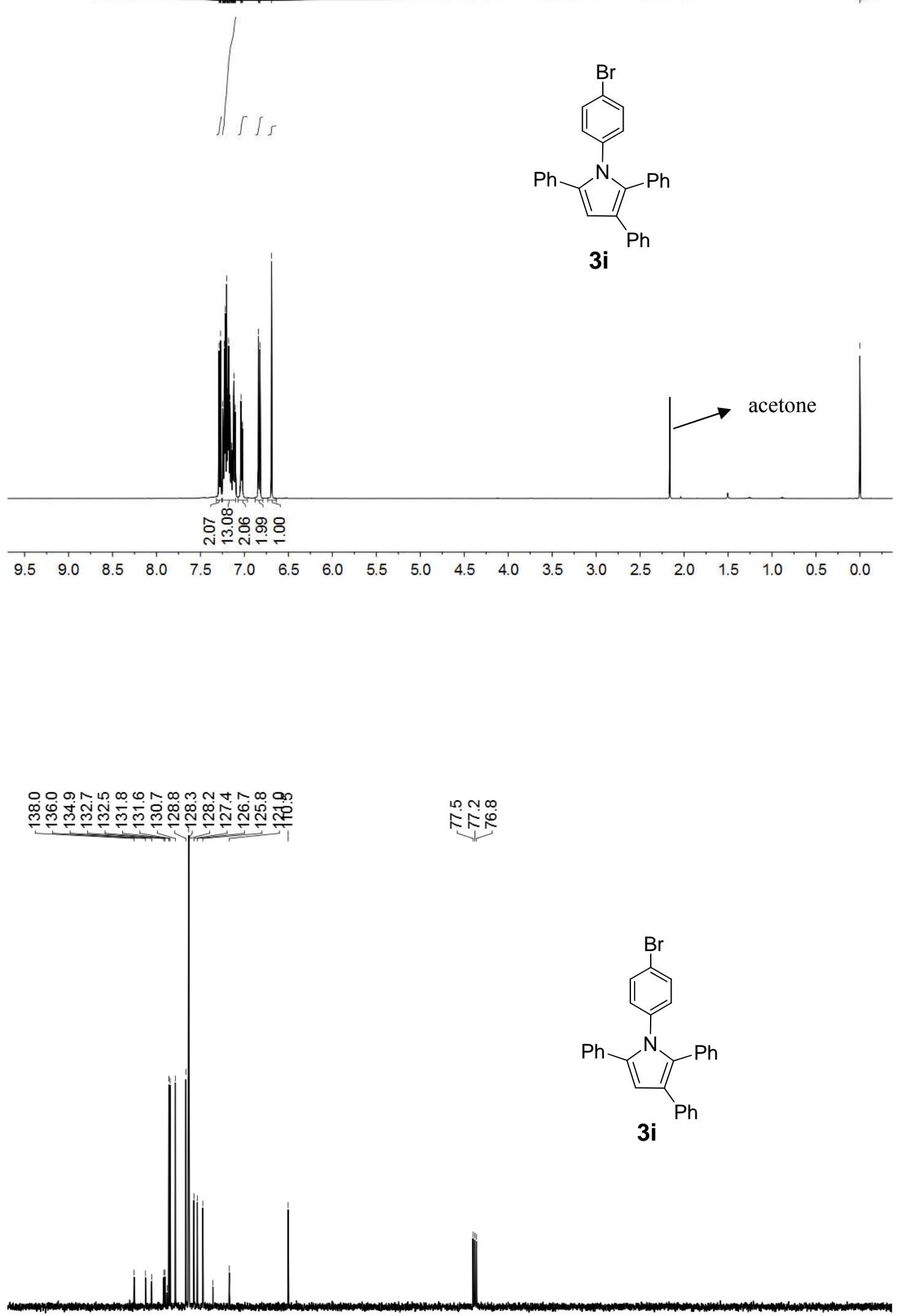

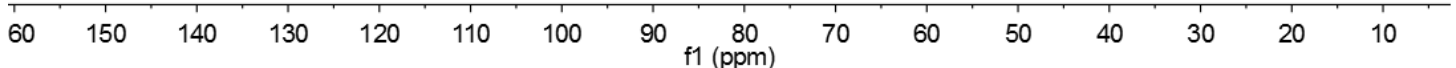




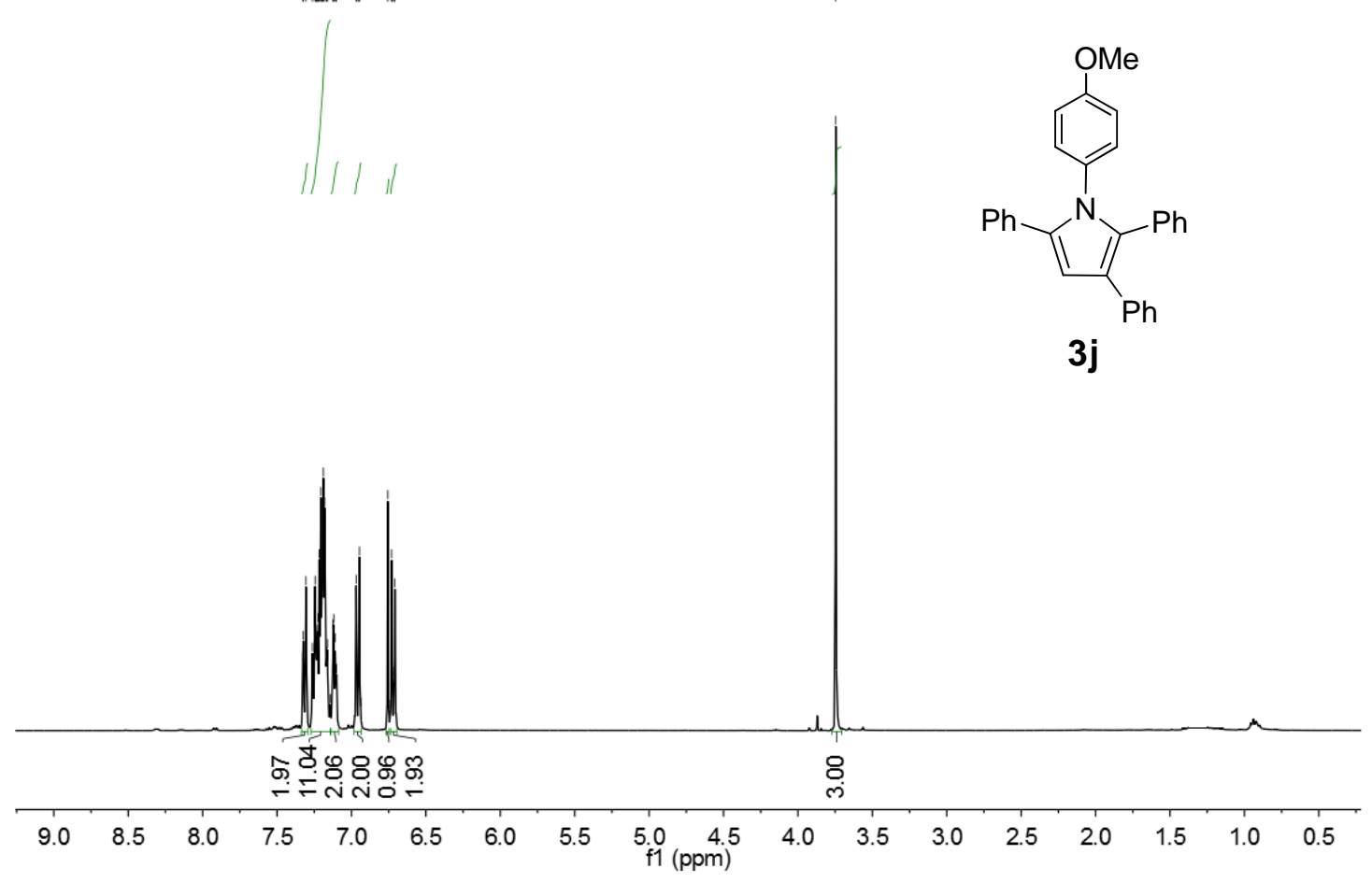

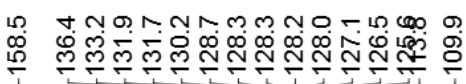

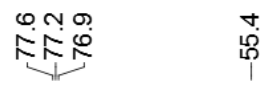

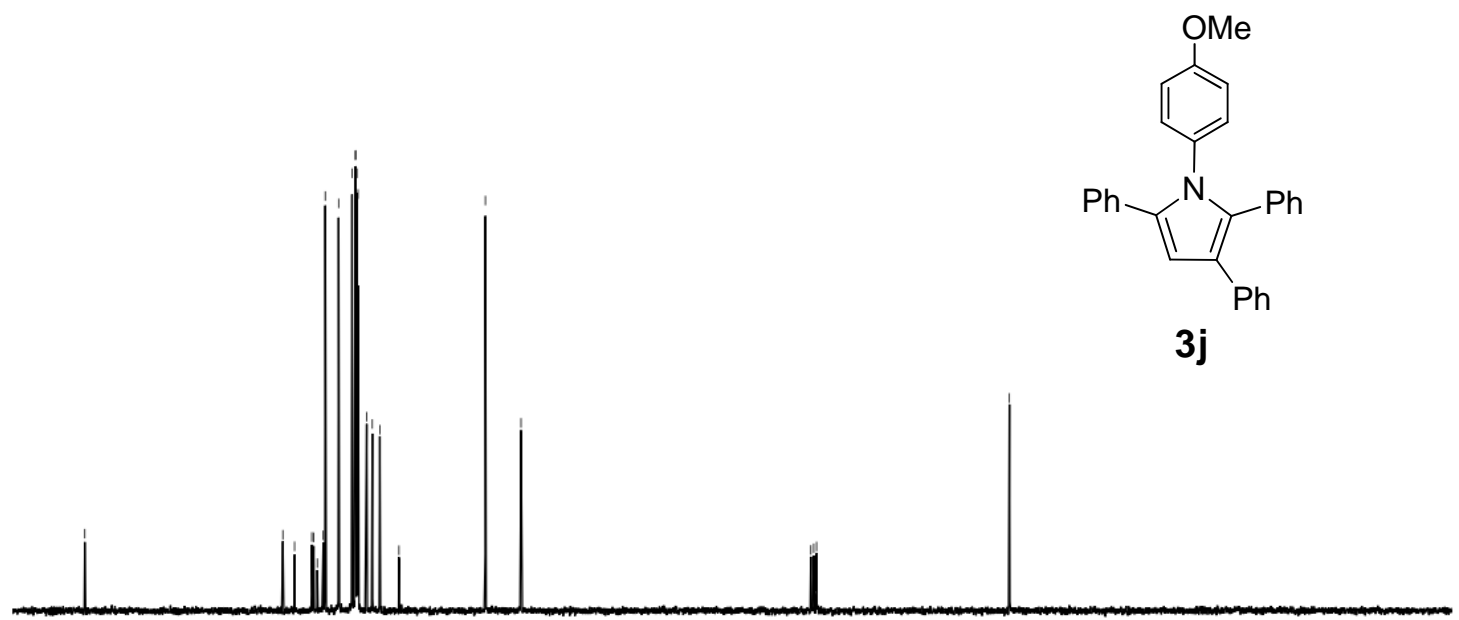

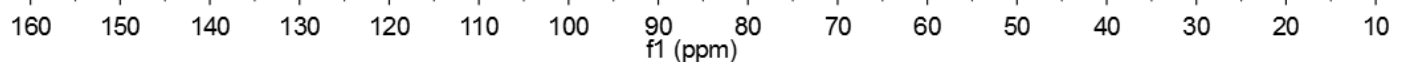



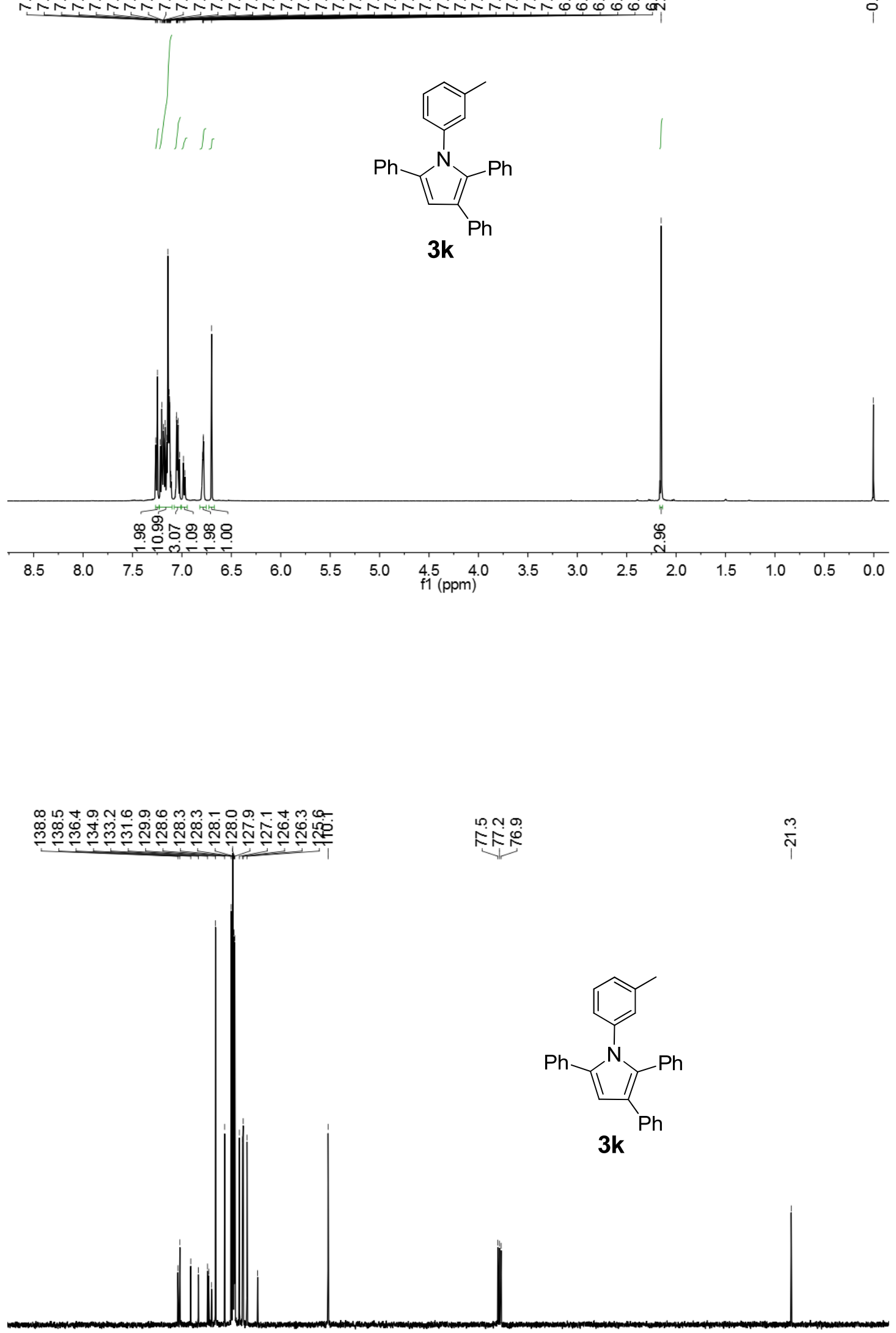

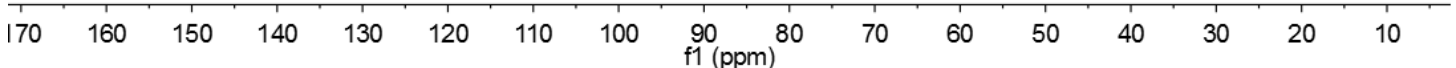



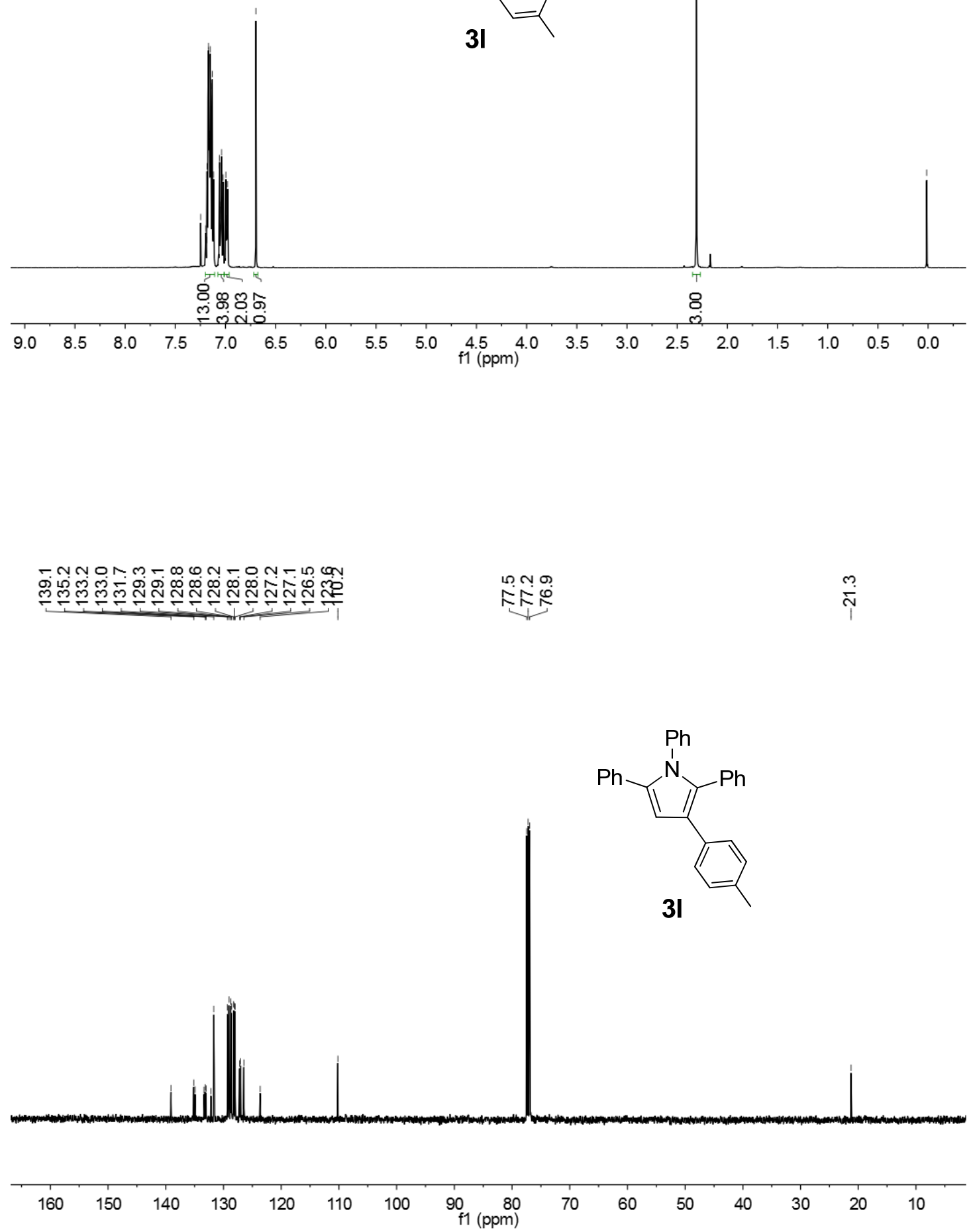


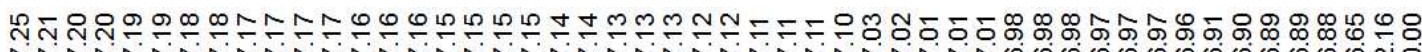

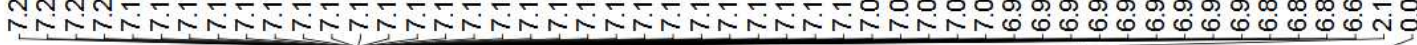
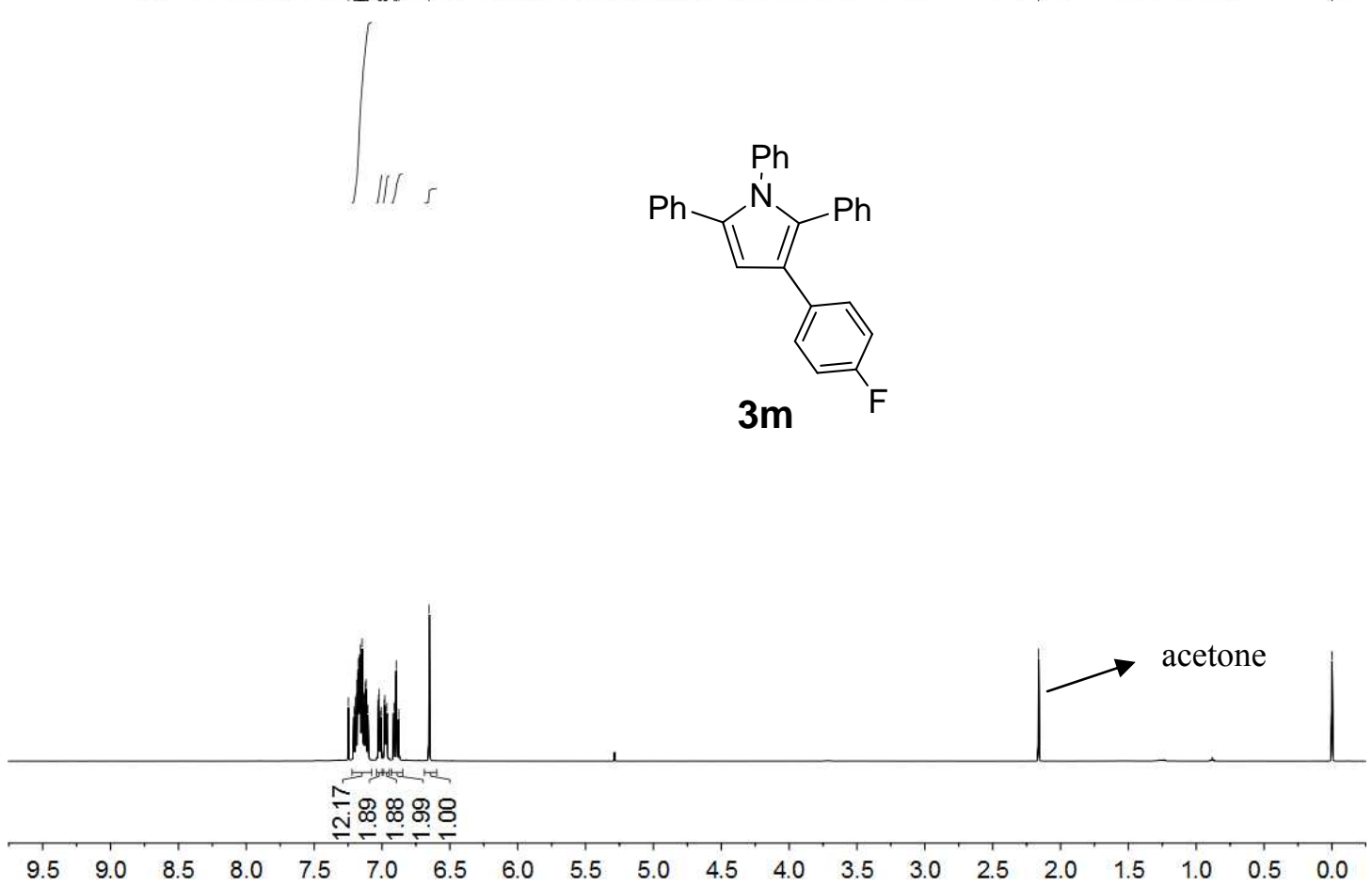

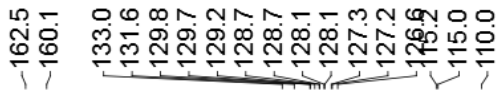

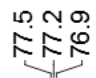

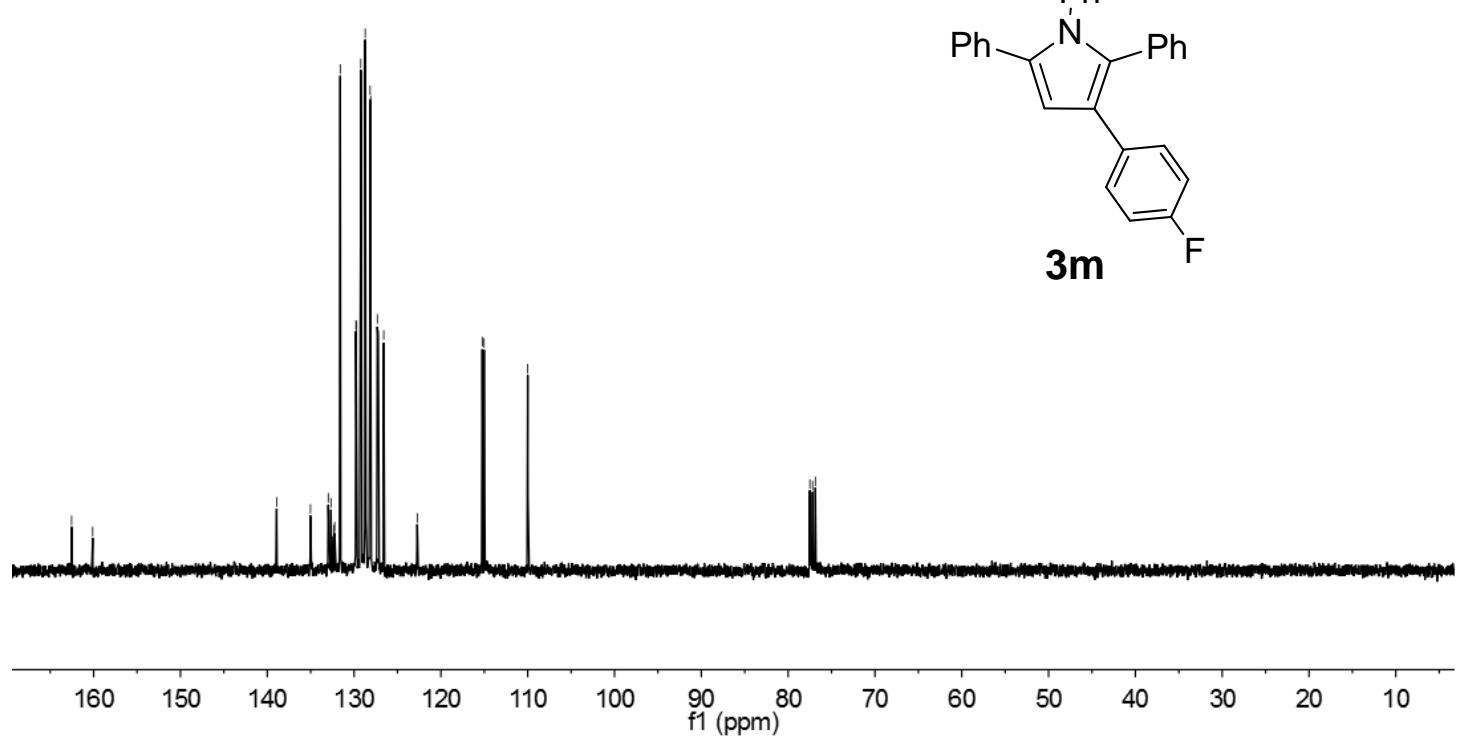



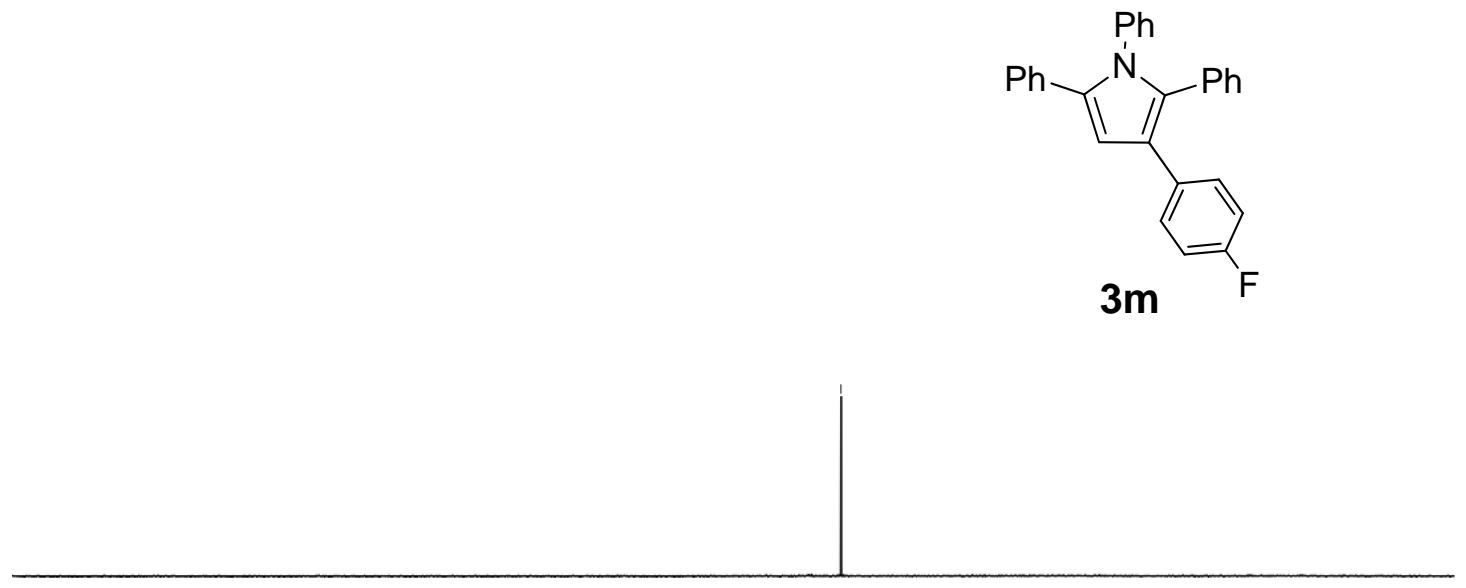


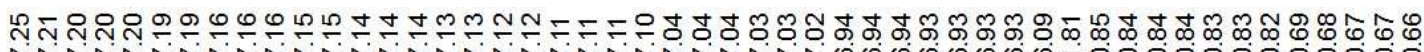

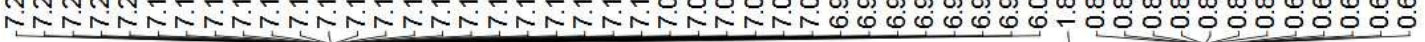
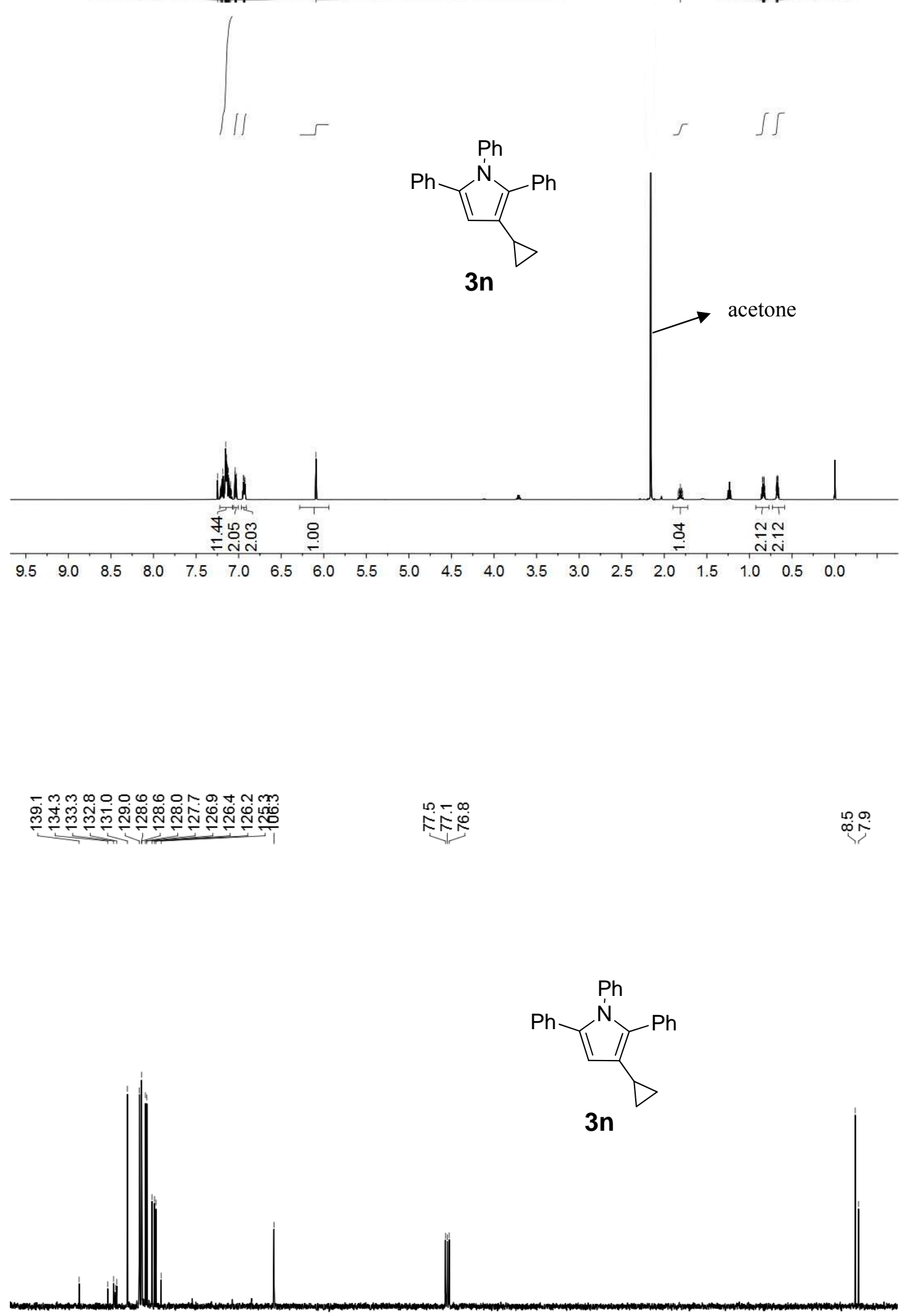

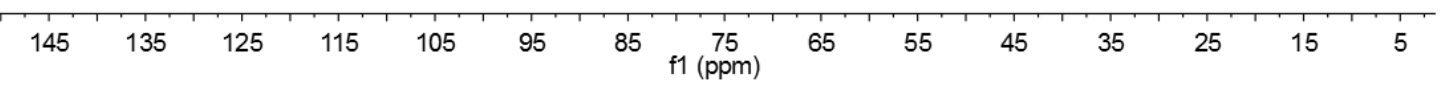




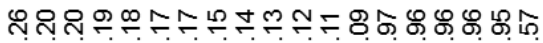

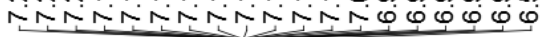
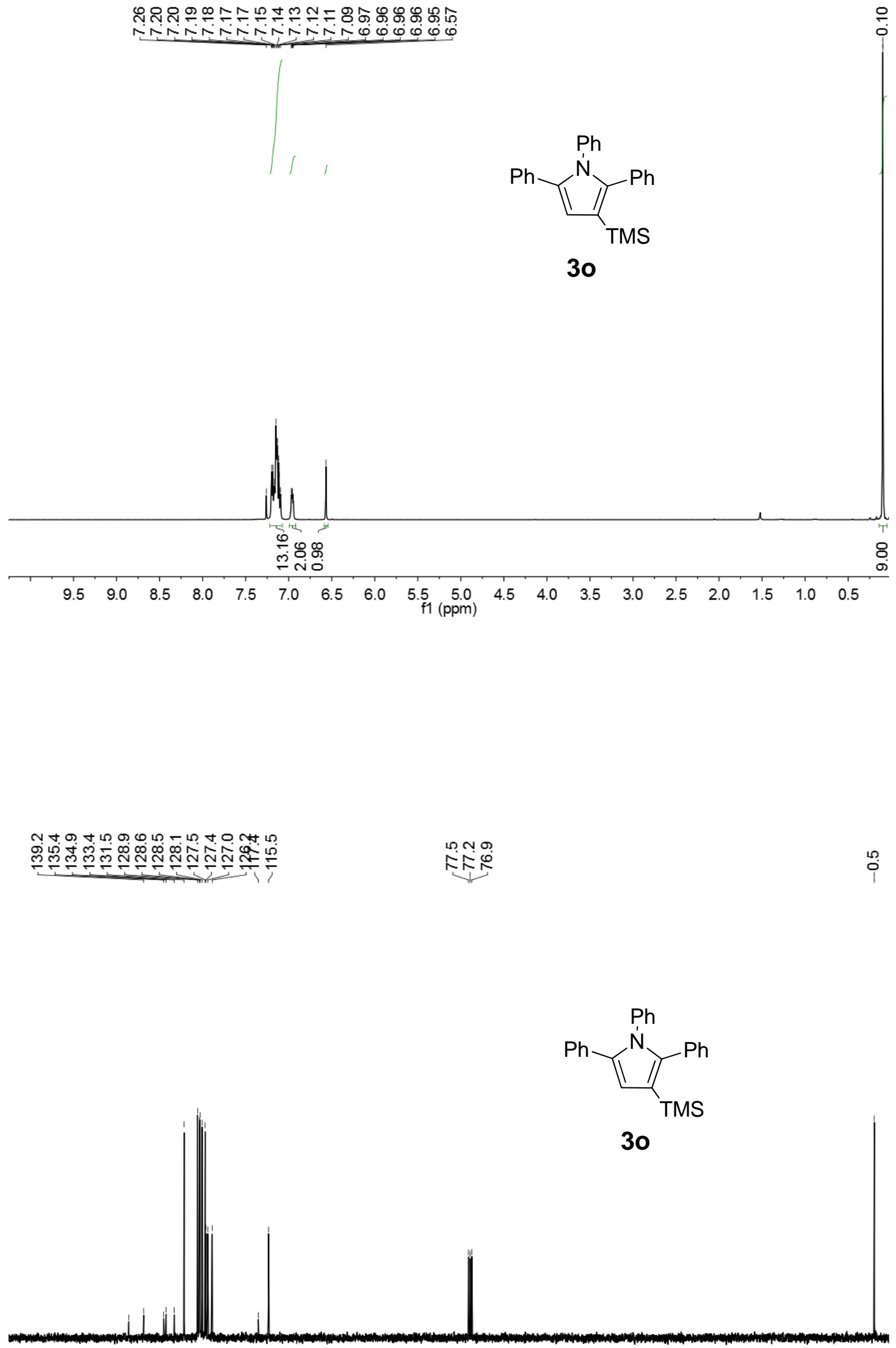
<smiles>CCCCc1cc(-c2ccccc2)n(-c2ccccc2)c1-c1ccccc1</smiles>
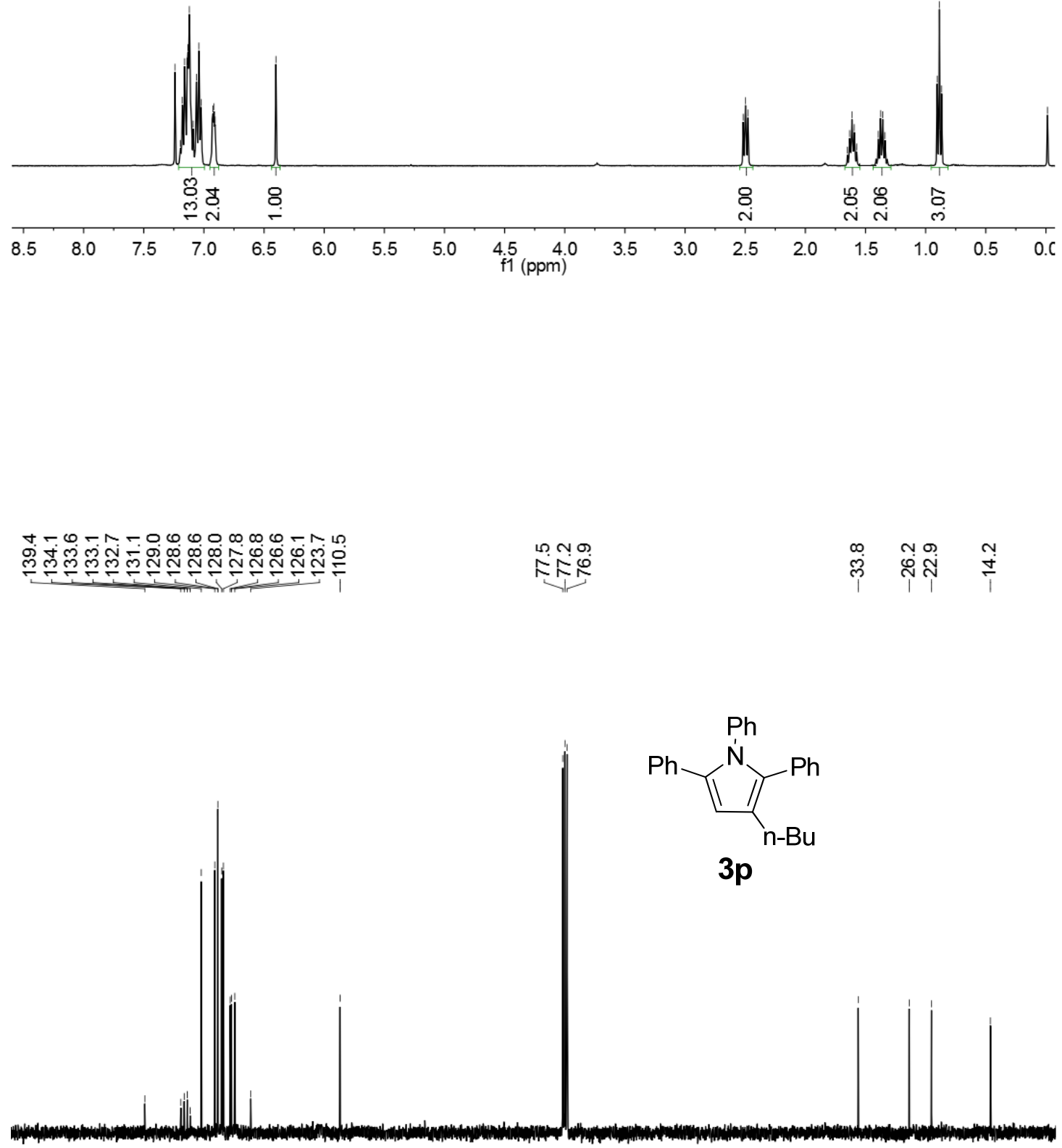


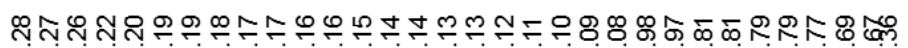

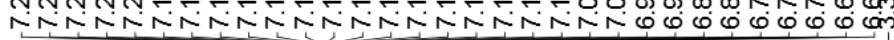

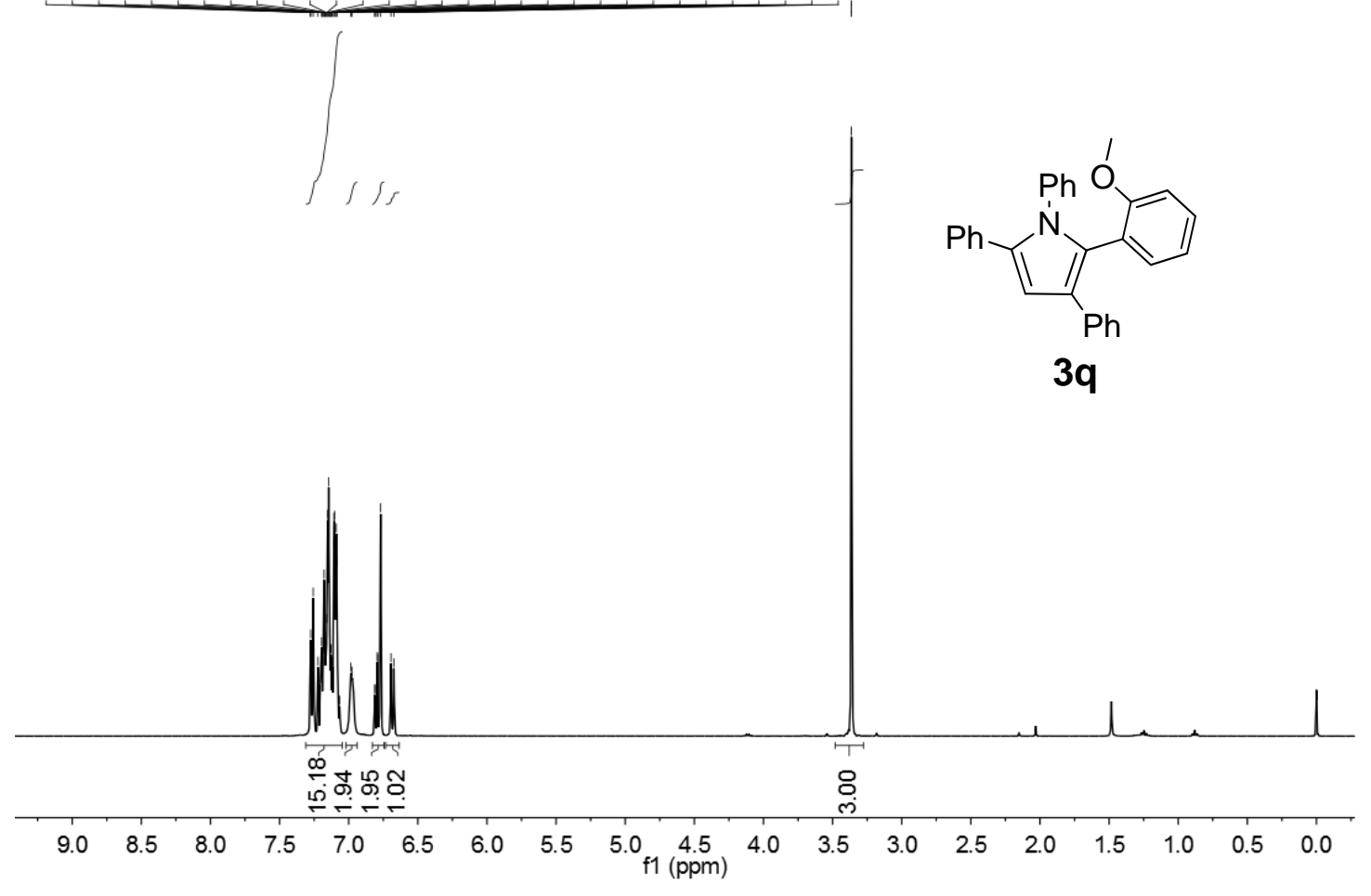

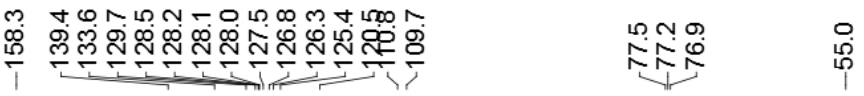

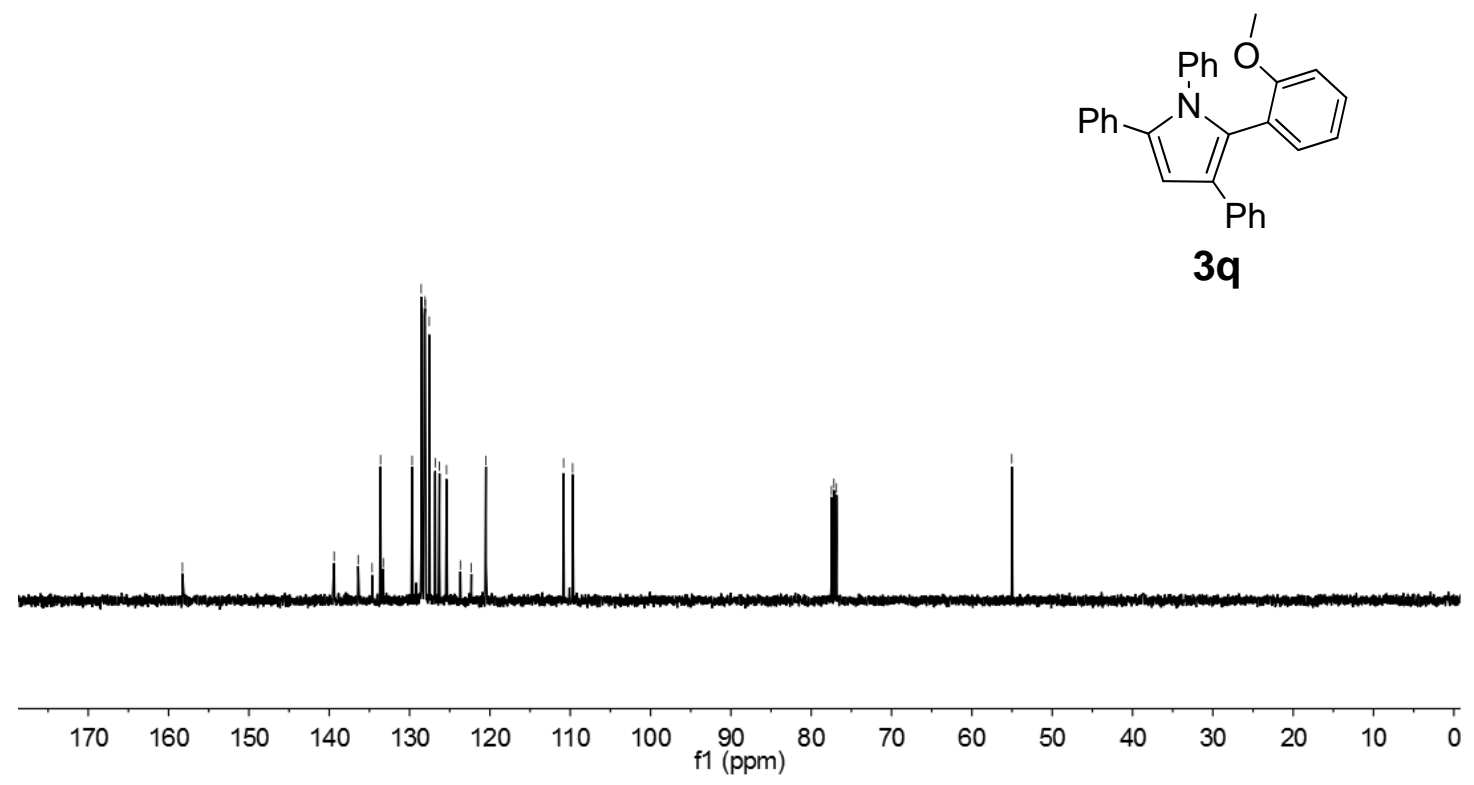




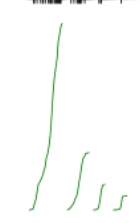<smiles>Cc1cccc(-c2[nH]c(-c3ccccc3)cc2-c2ccccc2)c1</smiles>
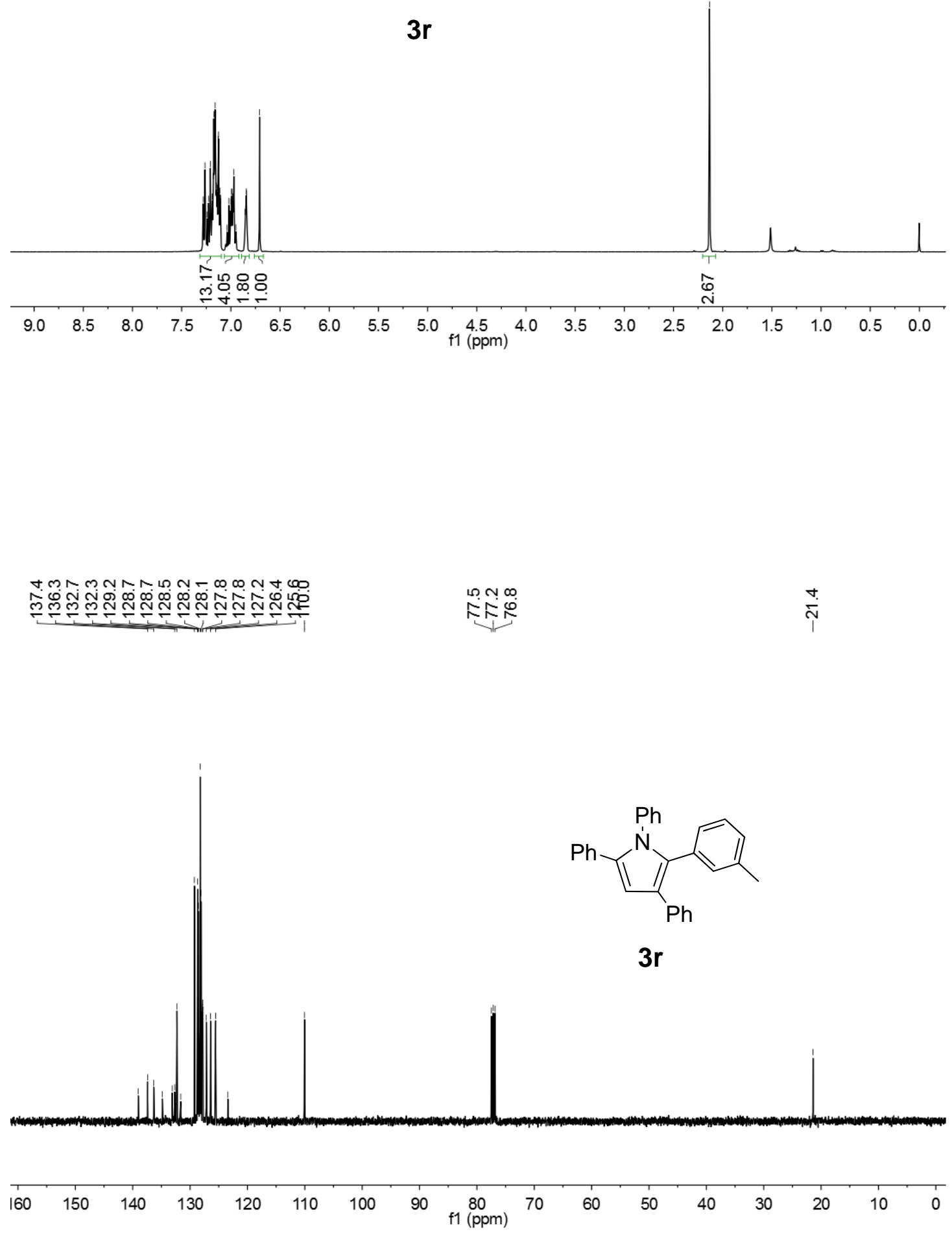


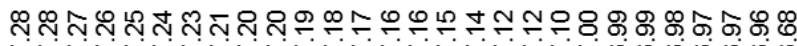

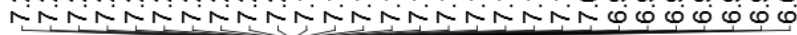
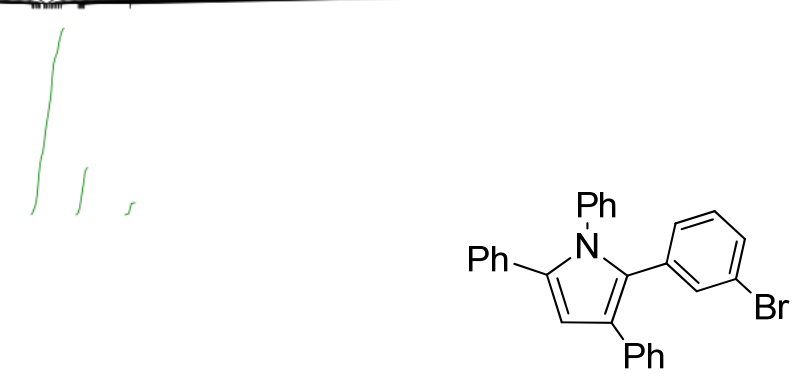

3s
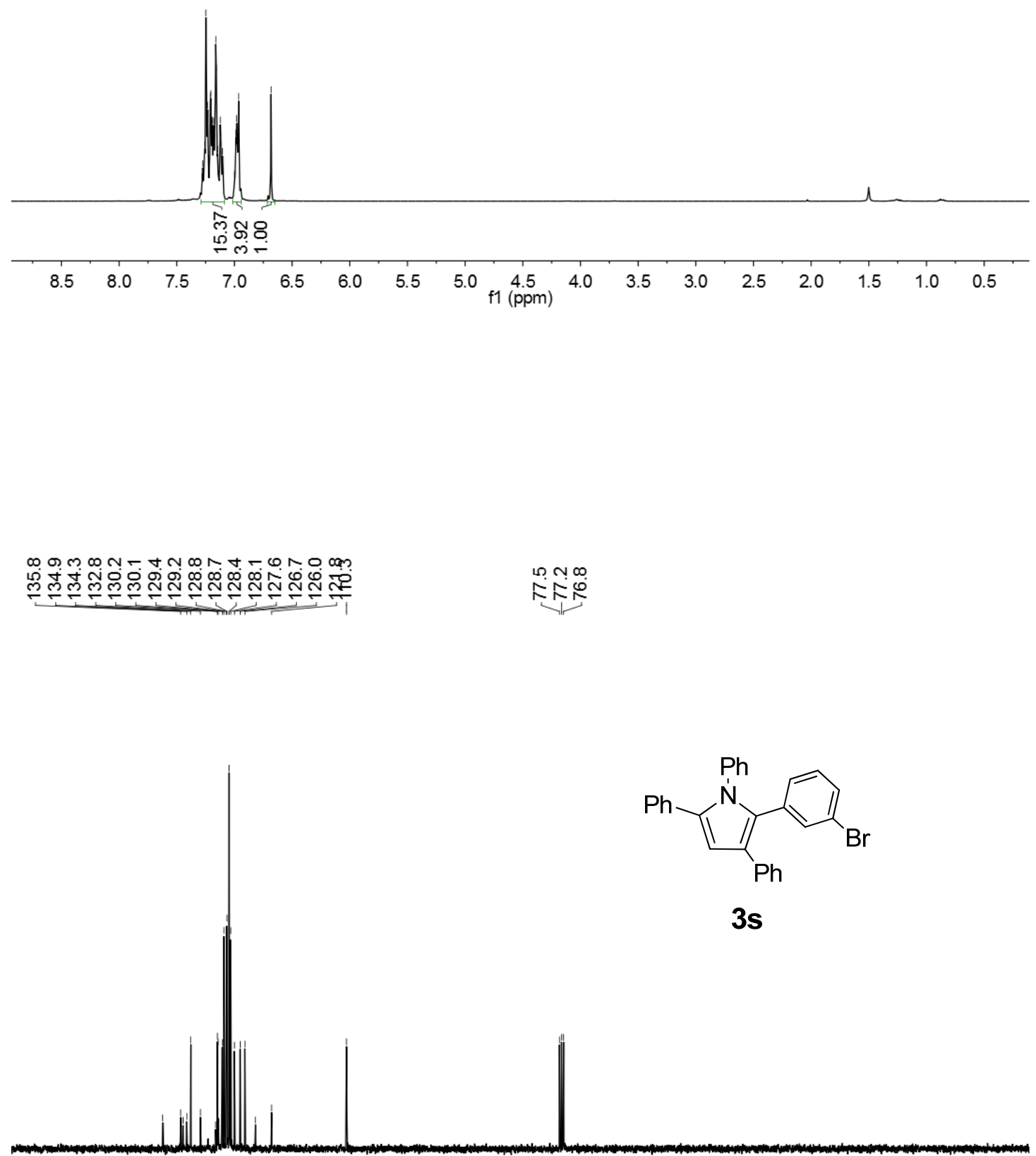

$\begin{array}{lllllllllllllll}160 & 150 & 140 & 130 & 120 & 110 & 100 & 90 \begin{array}{r}80 \\ \mathrm{f} 1(\mathrm{ppm})\end{array} & 70 & 60 & 50 & 40 & 30 & 20 & 10\end{array}$



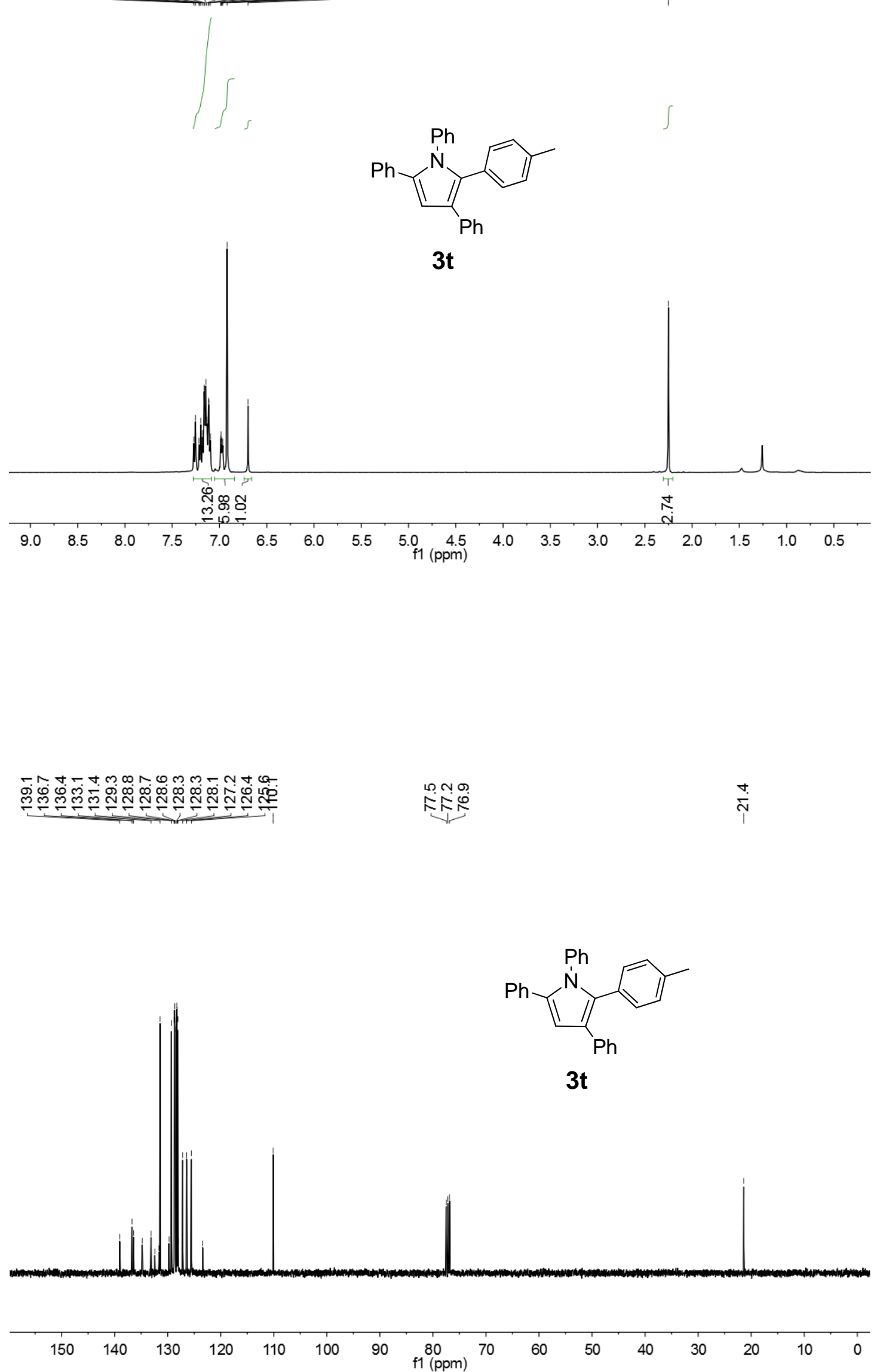

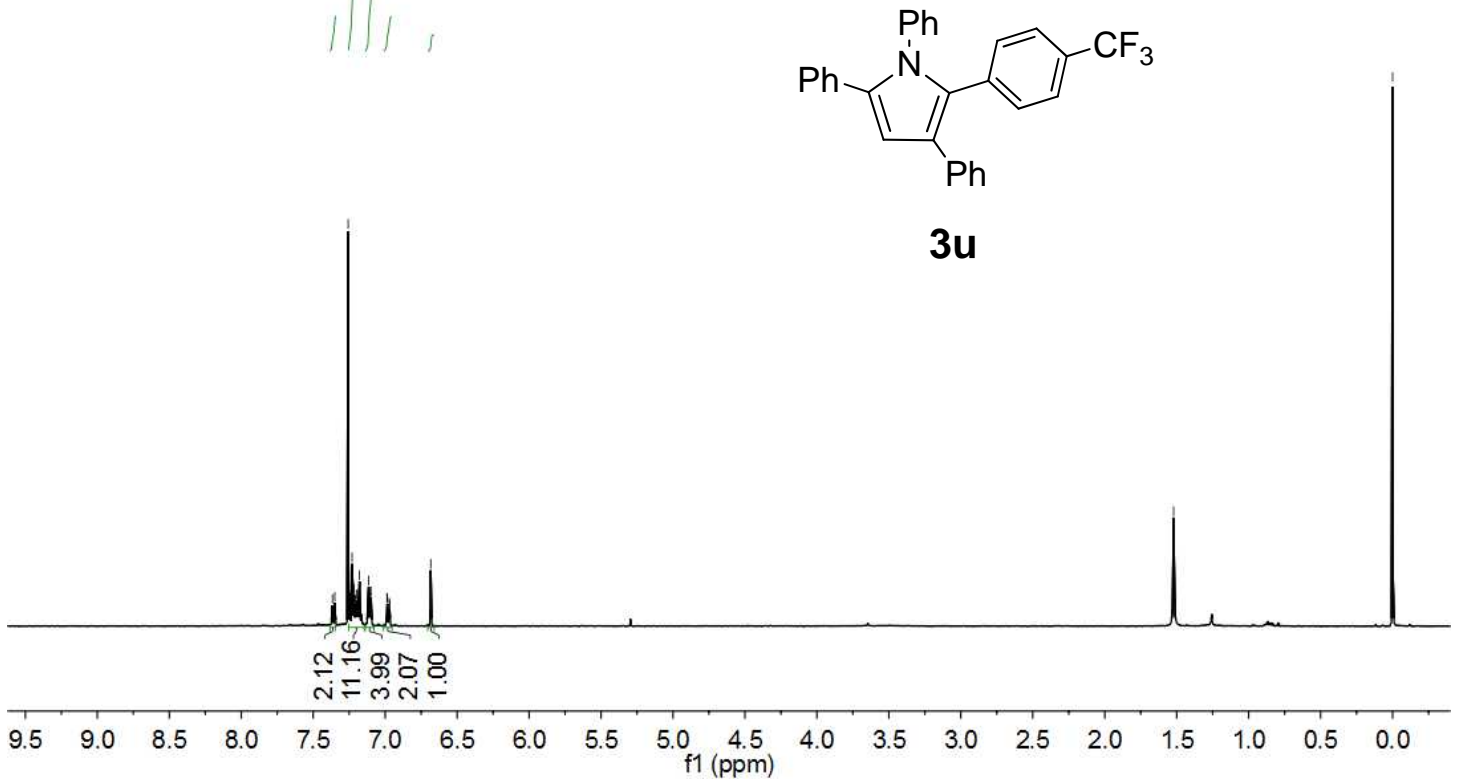

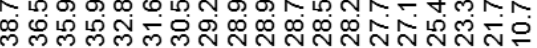

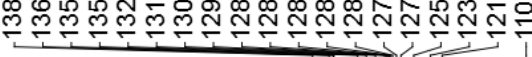

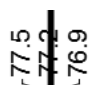

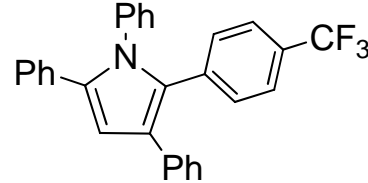

$3 u$ 
ণั่<smiles>FC(F)(F)c1ccc(-c2c(-c3ccccc3)cc(-c3ccccc3)n2-c2ccccc2)cc1</smiles>

$3 u$

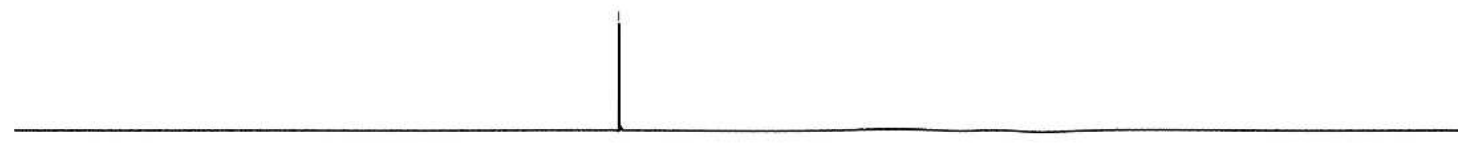

\begin{tabular}{llllllllllllllll}
\hline 120 & 80 & 60 & 40 & 20 & 0 & -20 & -40 & -60 & -80 & -100 & -140 & -180 & -220 & -260 & -300
\end{tabular} 
กิกฺุ
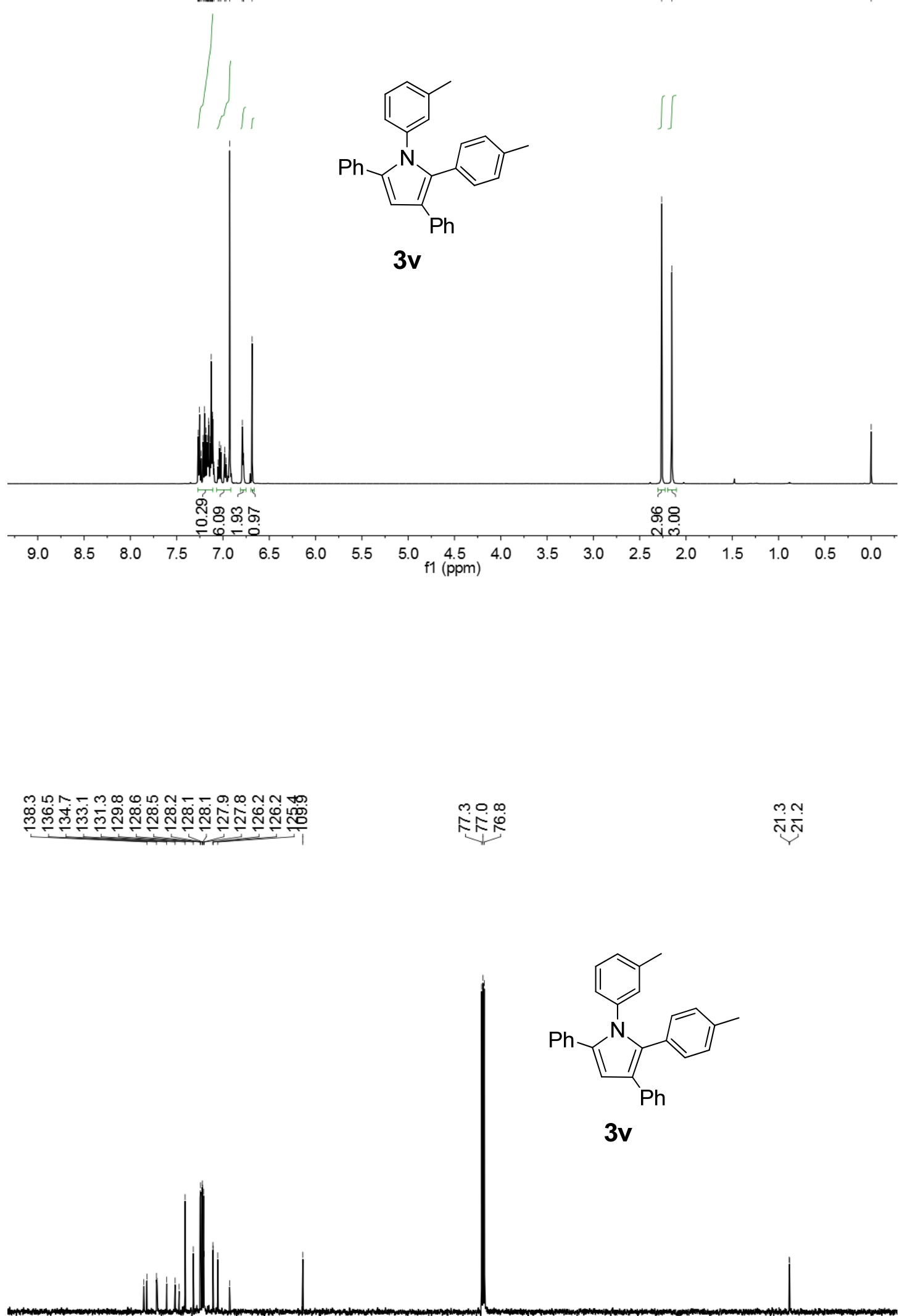

160 150 130 120 110100 $90 \begin{array}{r}80 \\ \mathrm{f} 1(\mathrm{ppm})\end{array}$ 

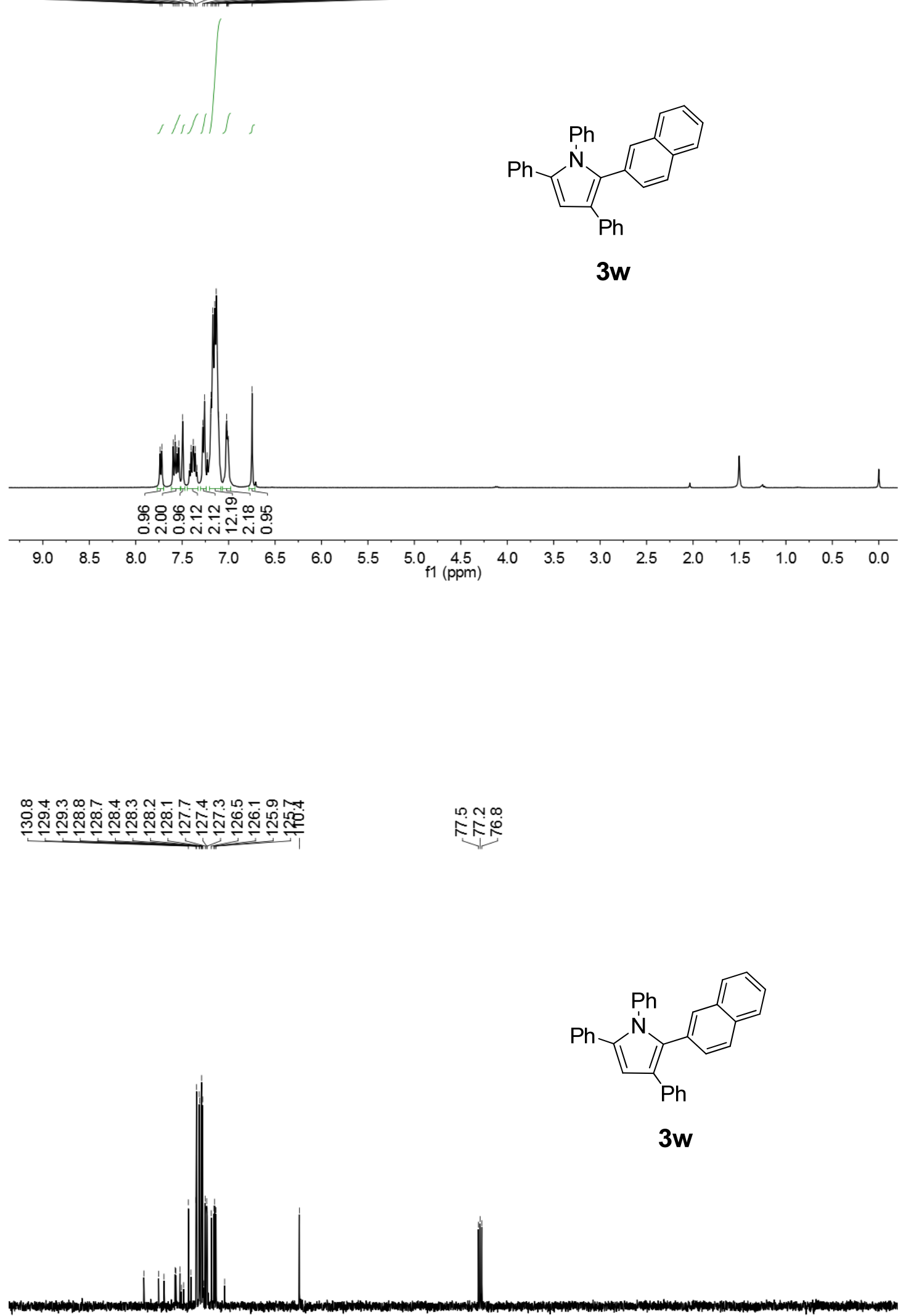<smiles>c1ccc(-c2cc(-c3ccccc3)n(-c3ccccc3)c2-c2ccc3ccccc3c2)cc1</smiles>

$3 w$ 

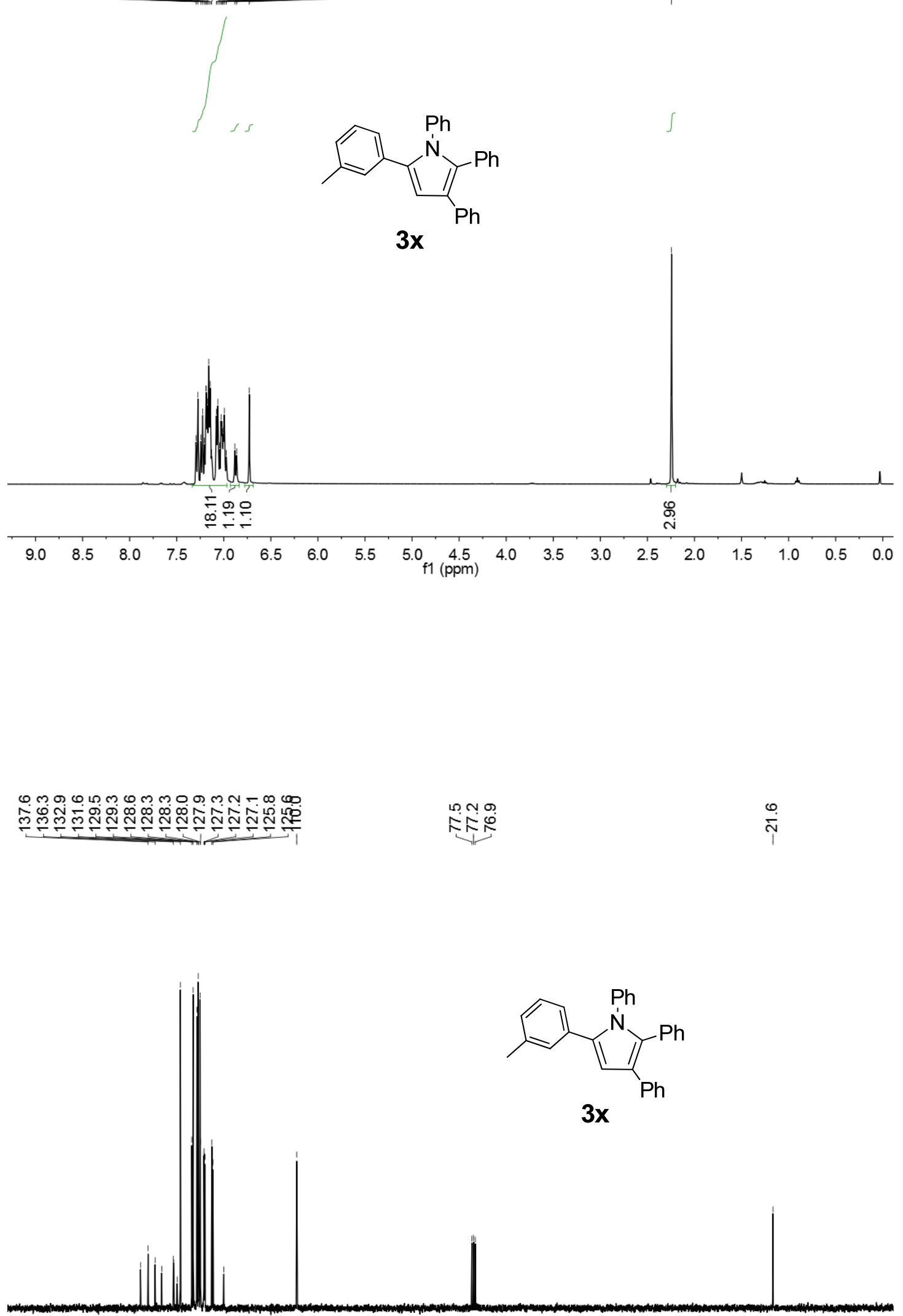

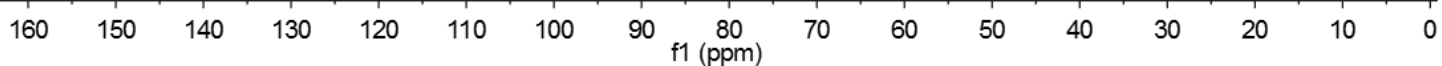




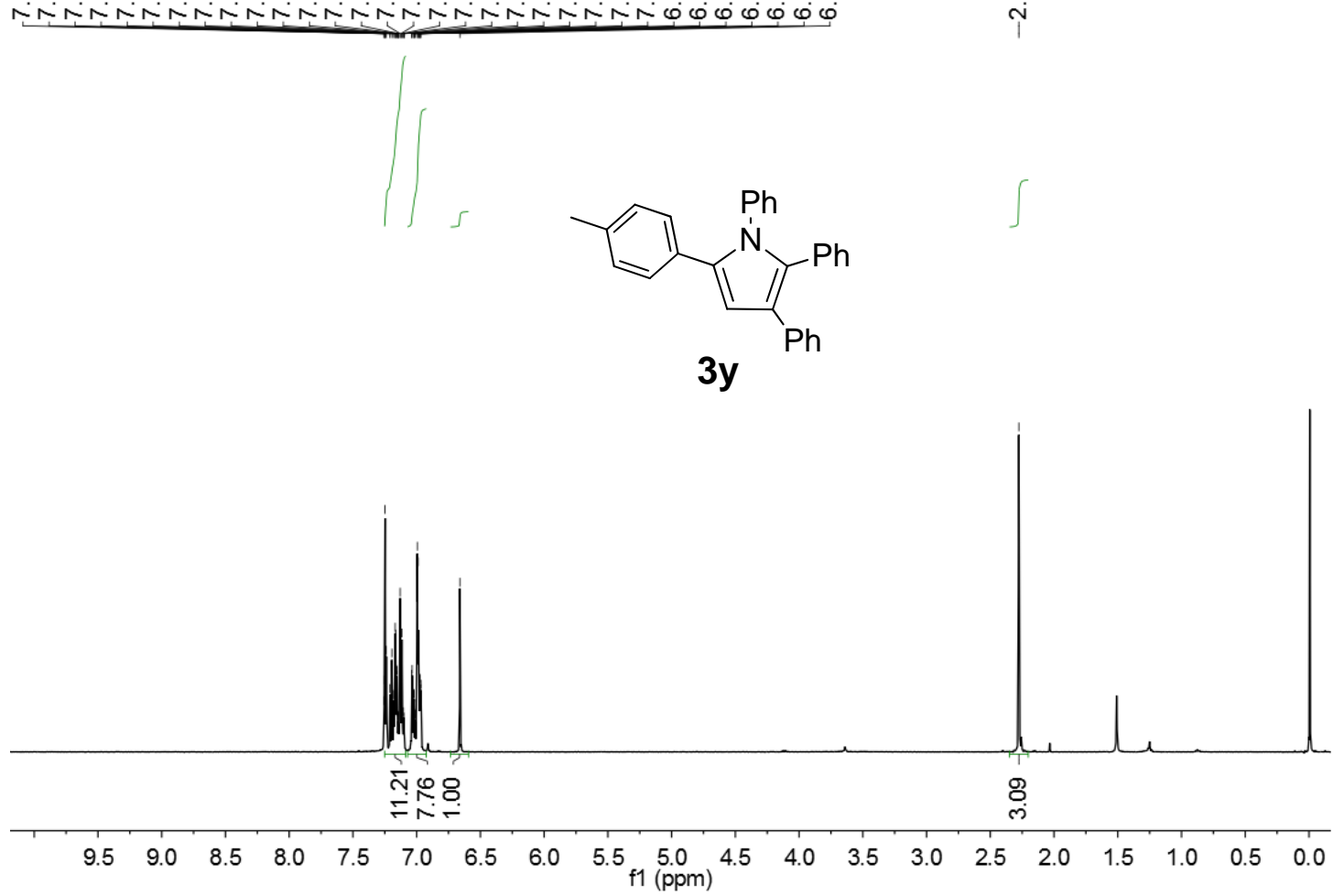<smiles>Cc1ccc(-c2cc(-c3ccccc3)c(-c3ccccc3)n2-c2ccccc2)cc1</smiles>

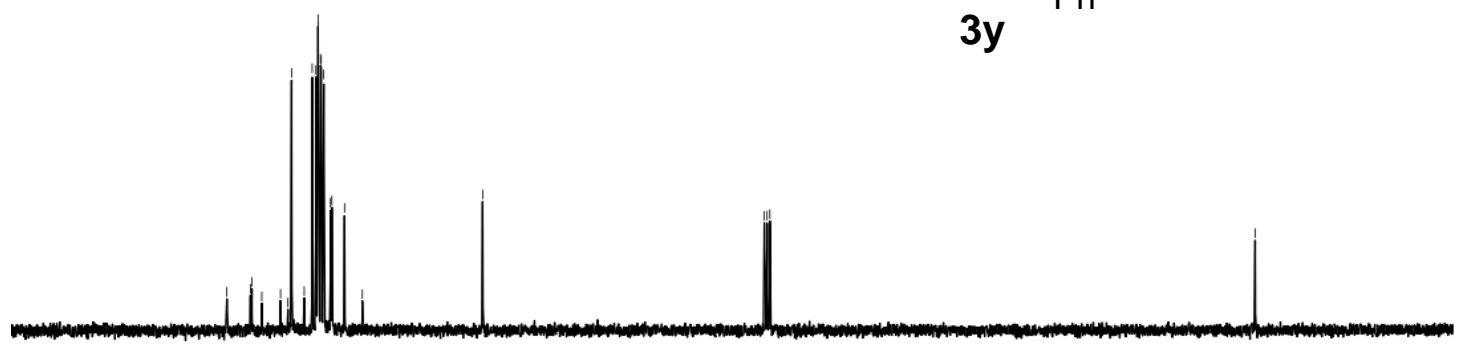

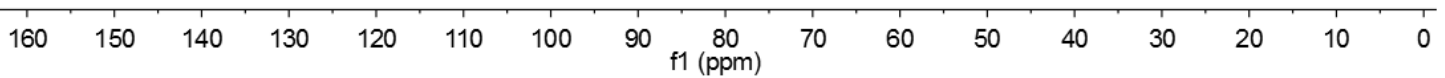




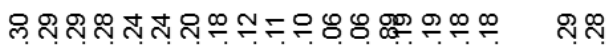

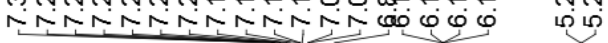

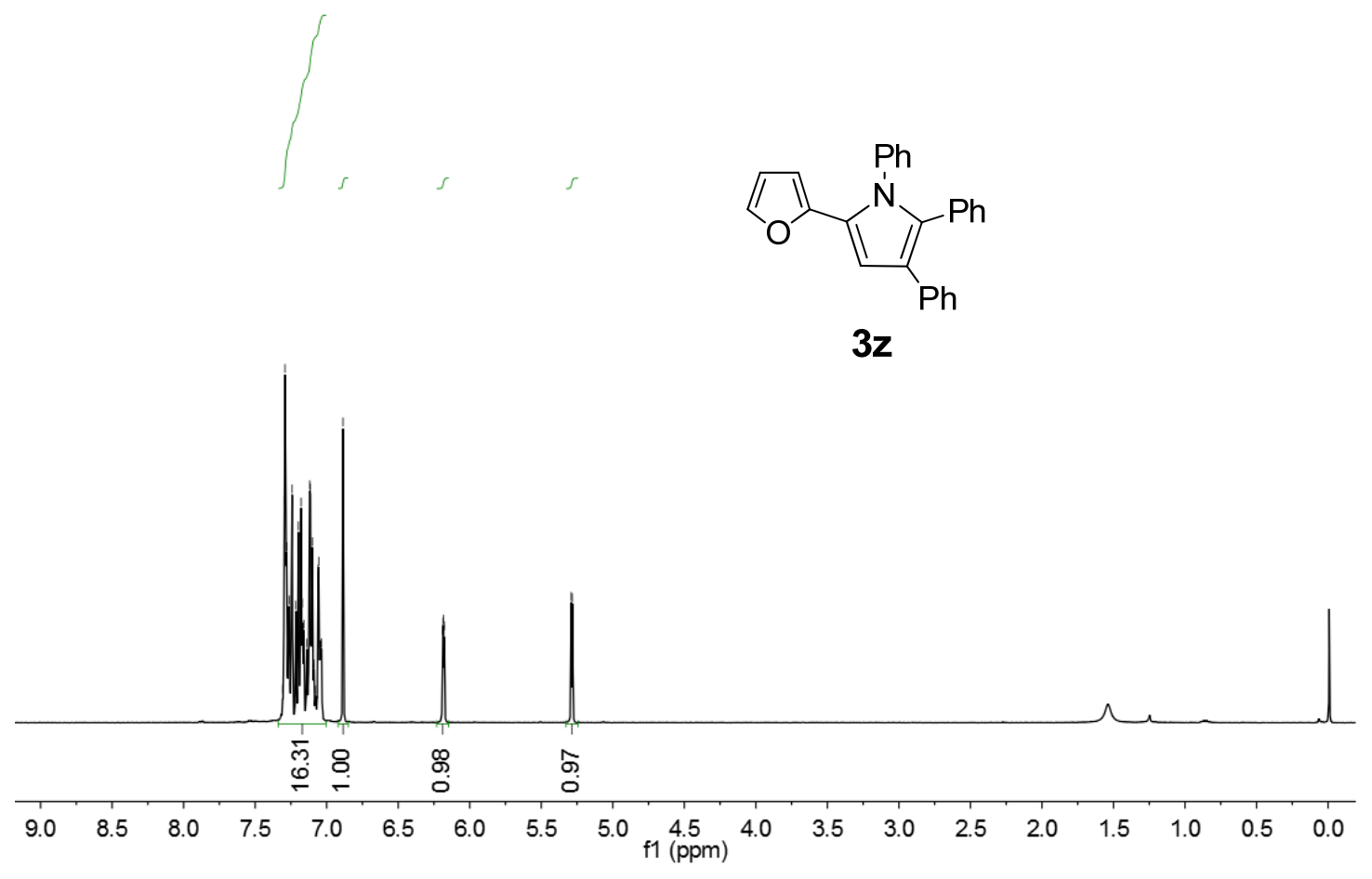

L

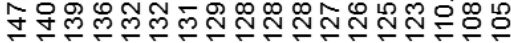

№
NNo

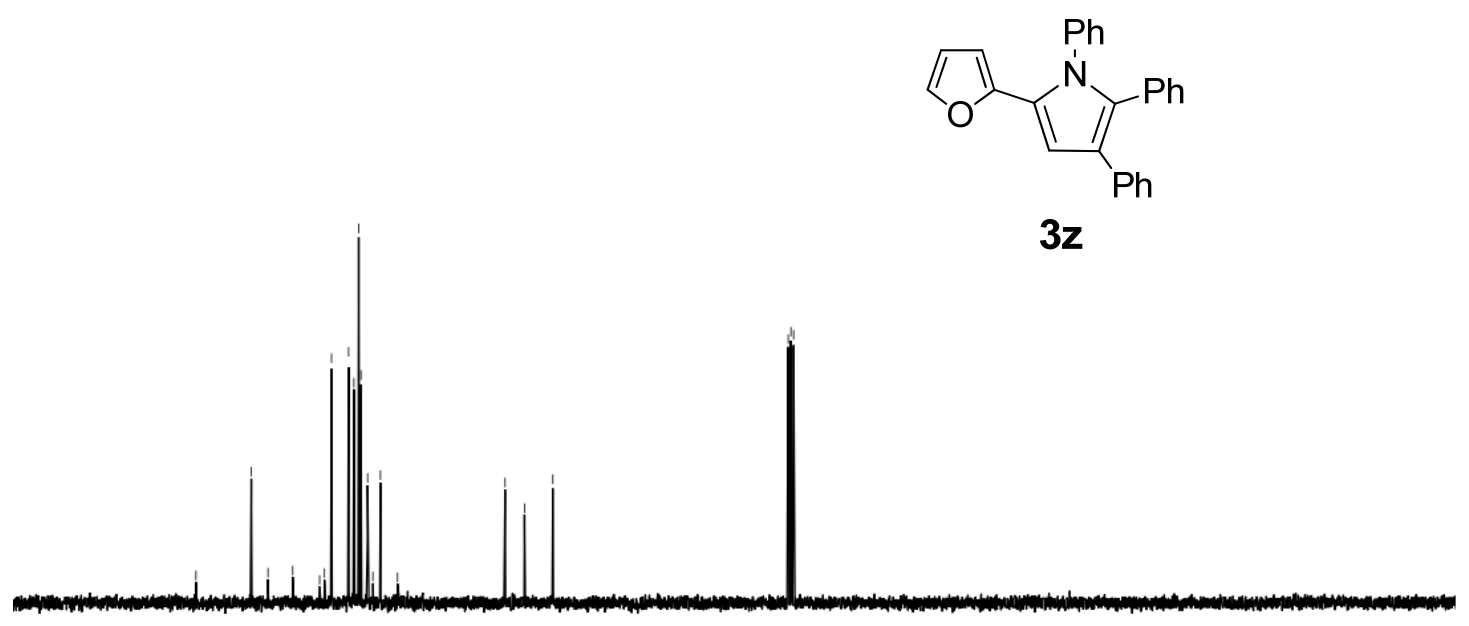

160

130

110

100

$90 \quad 80$

70

$60 \quad 50$

4

$\begin{array}{llll}30 & 20 & 10 & 0\end{array}$ 


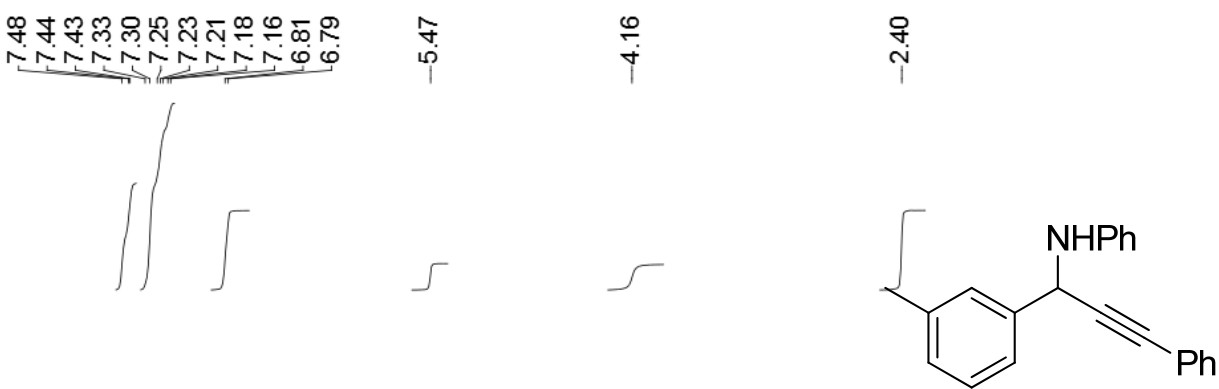

4b
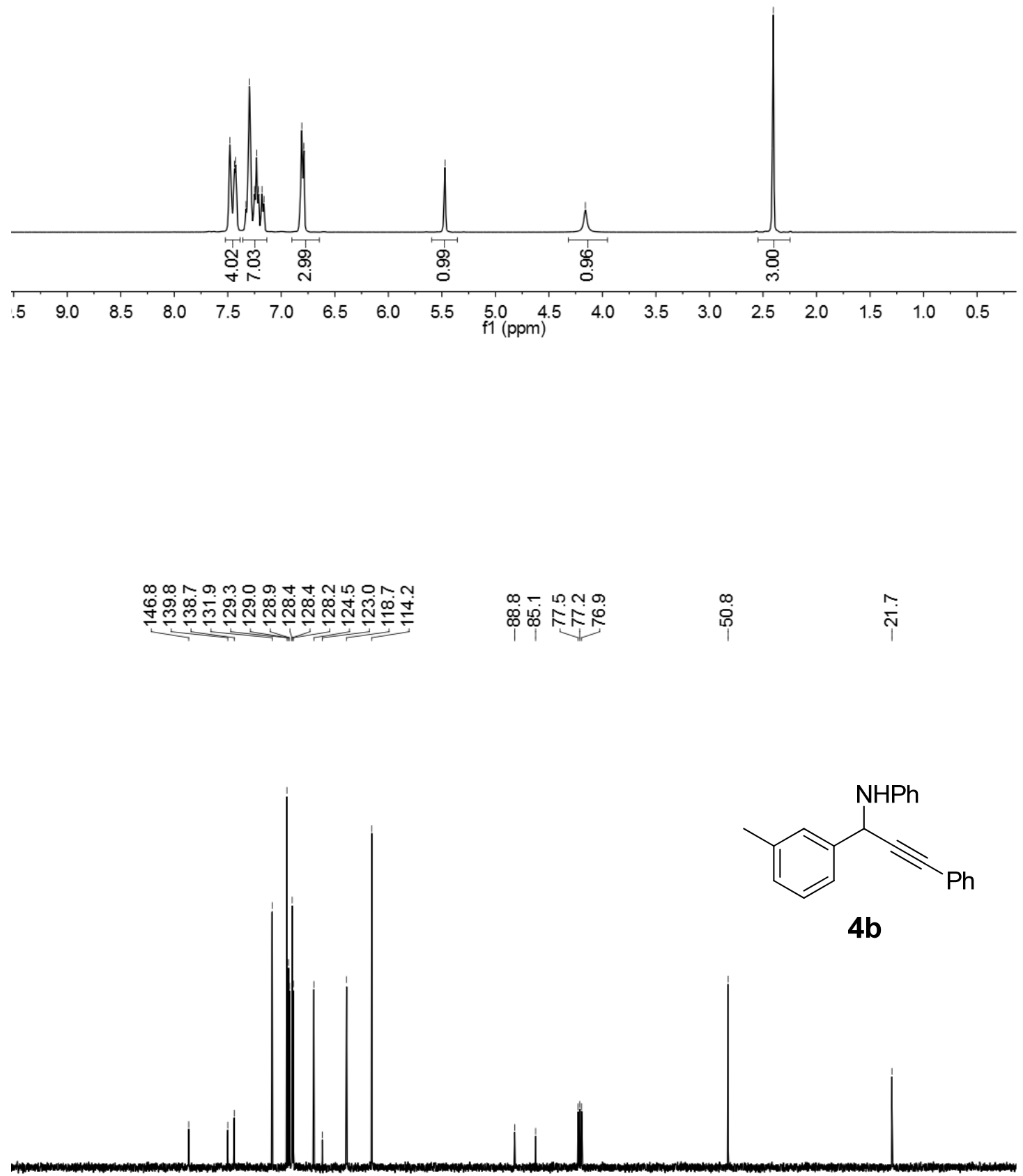

$\begin{array}{llllllllllllllllll}170 & 160 & 150 & 140 & 130 & 120 & 110 & 100 & \begin{array}{l}90 \\ \mathrm{f} 1(\mathrm{ppm})\end{array} & 80 & 70 & 60 & 50 & 40 & 30 & 20 & 10 & \mathrm{C}\end{array}$ 


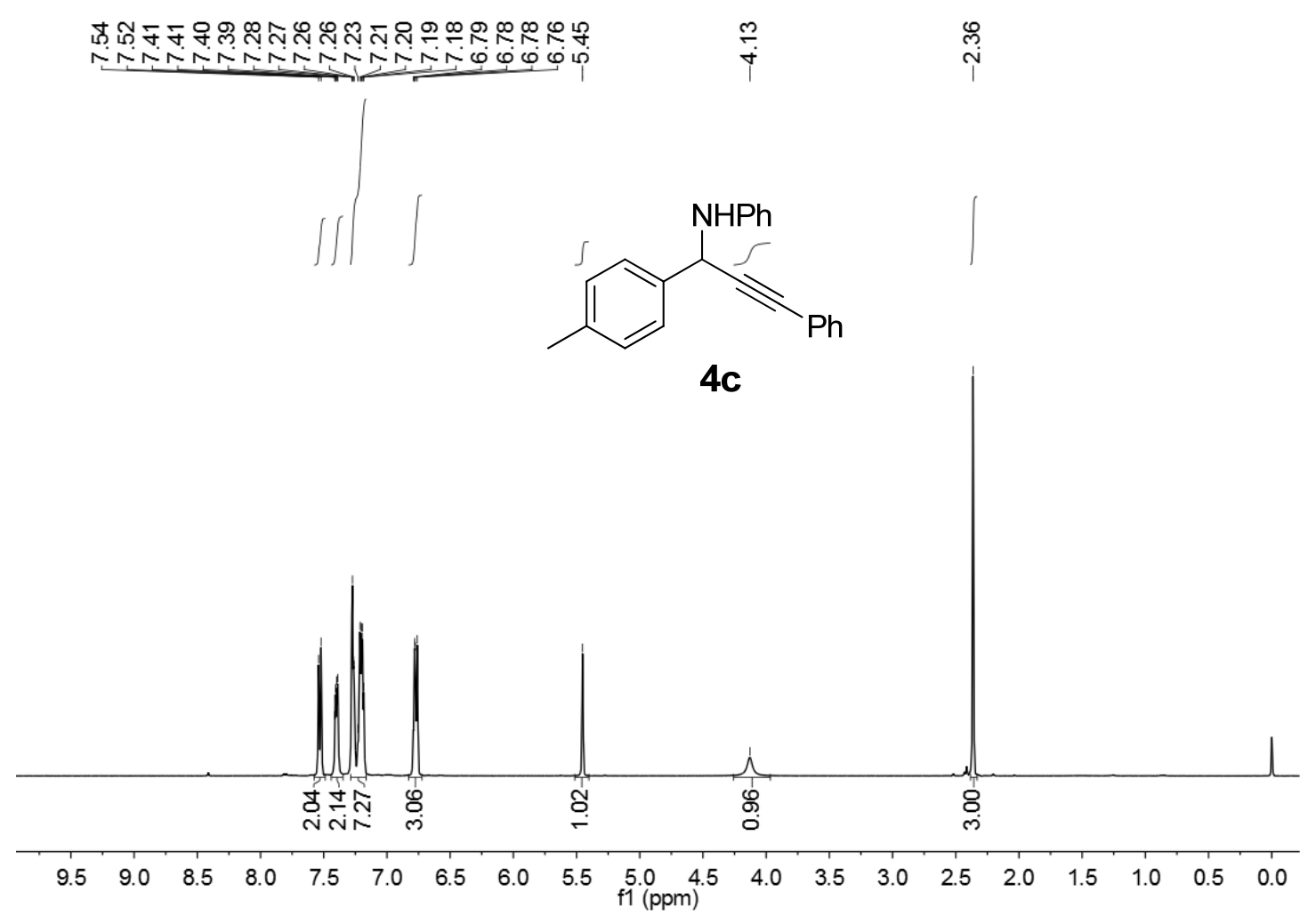

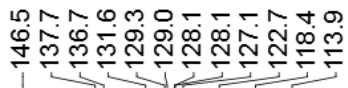

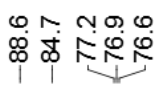

$\stackrel{m}{\circ}$

$\stackrel{\circ}{i}$

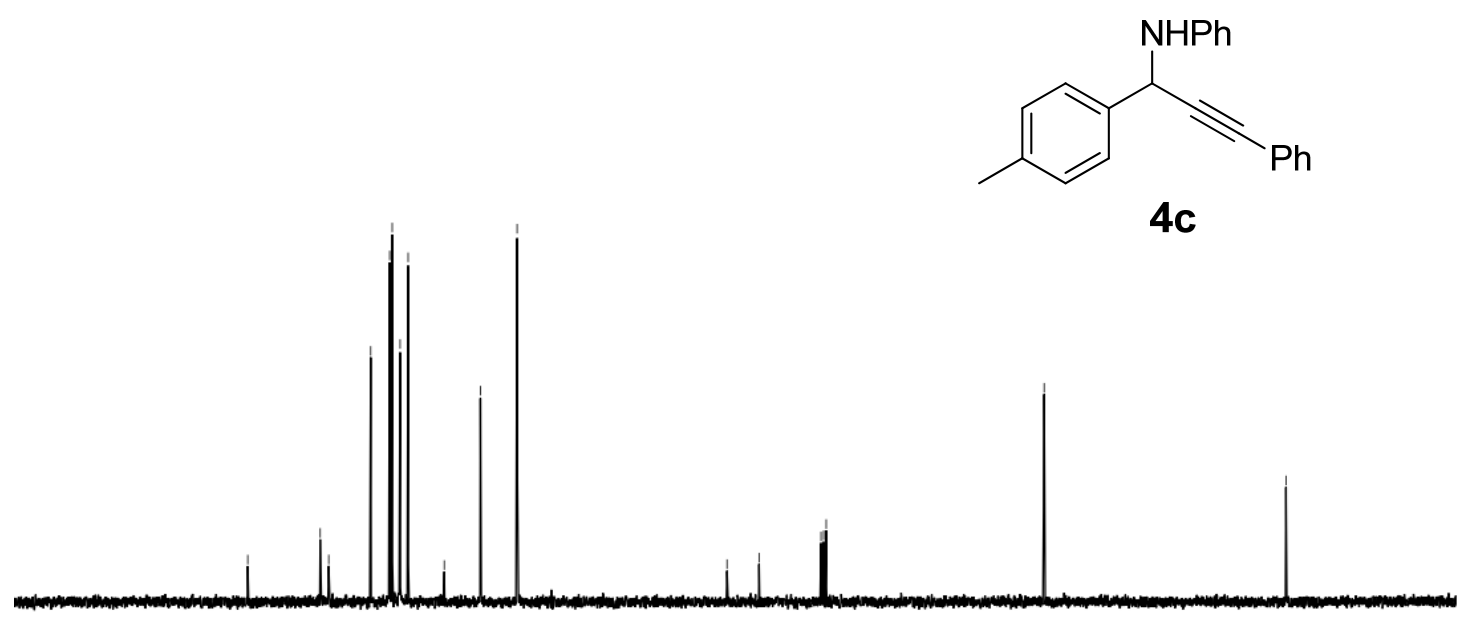

$\begin{array}{lllllllllllllllll}170 & 160 & 150 & 140 & 130 & 120 & 110 & 100 & \begin{array}{c}90 \\ \mathrm{f} 1(\mathrm{ppm})\end{array} & 80 & 70 & 60 & 50 & 40 & 30 & 20 & 10\end{array}$ 

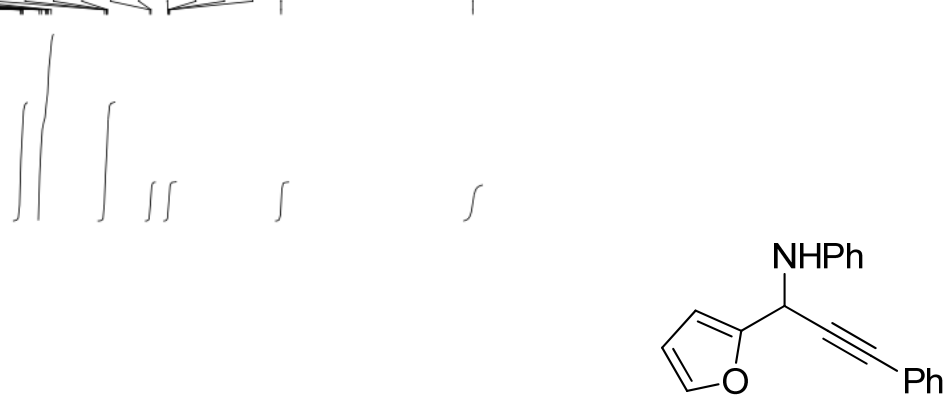

4d

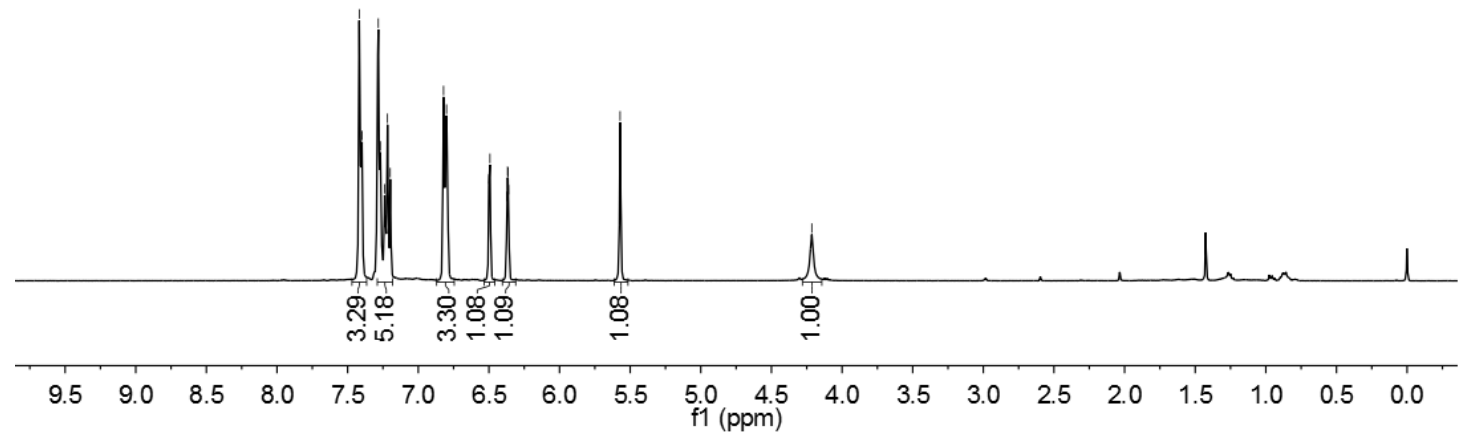

तิตู

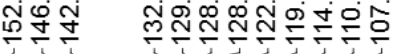

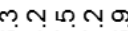

高年少

กี

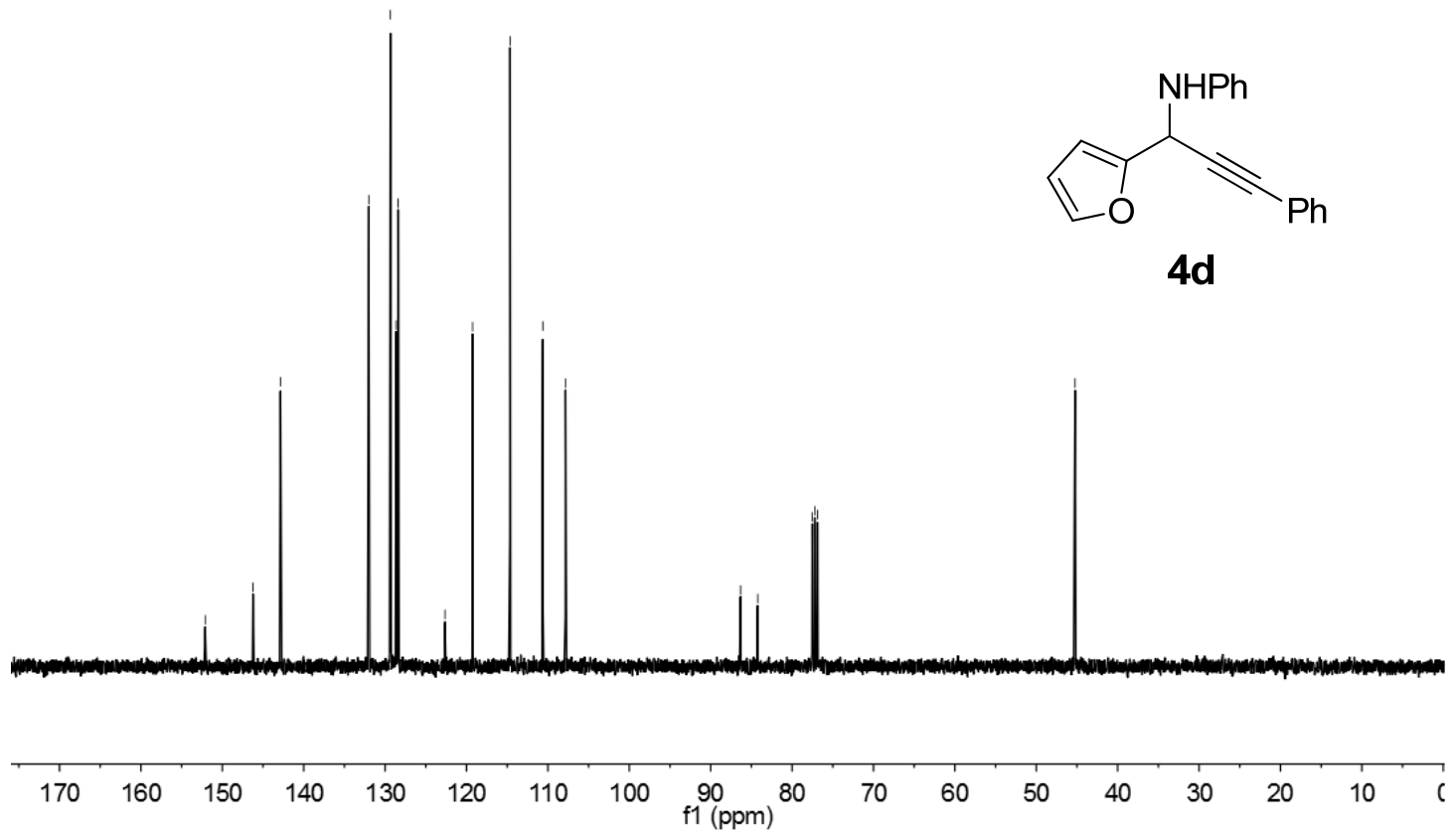




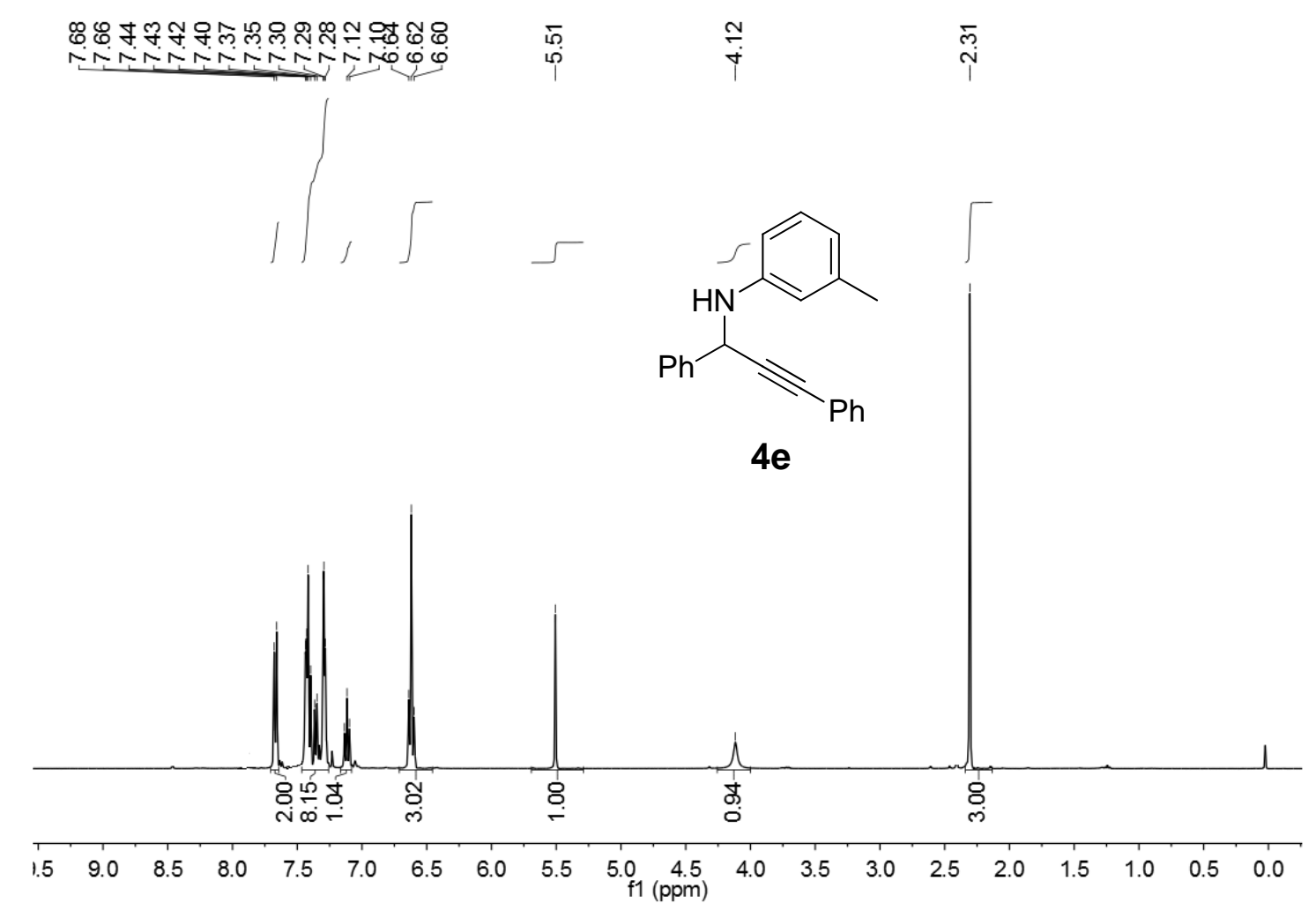

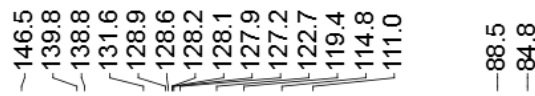

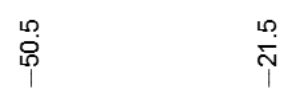
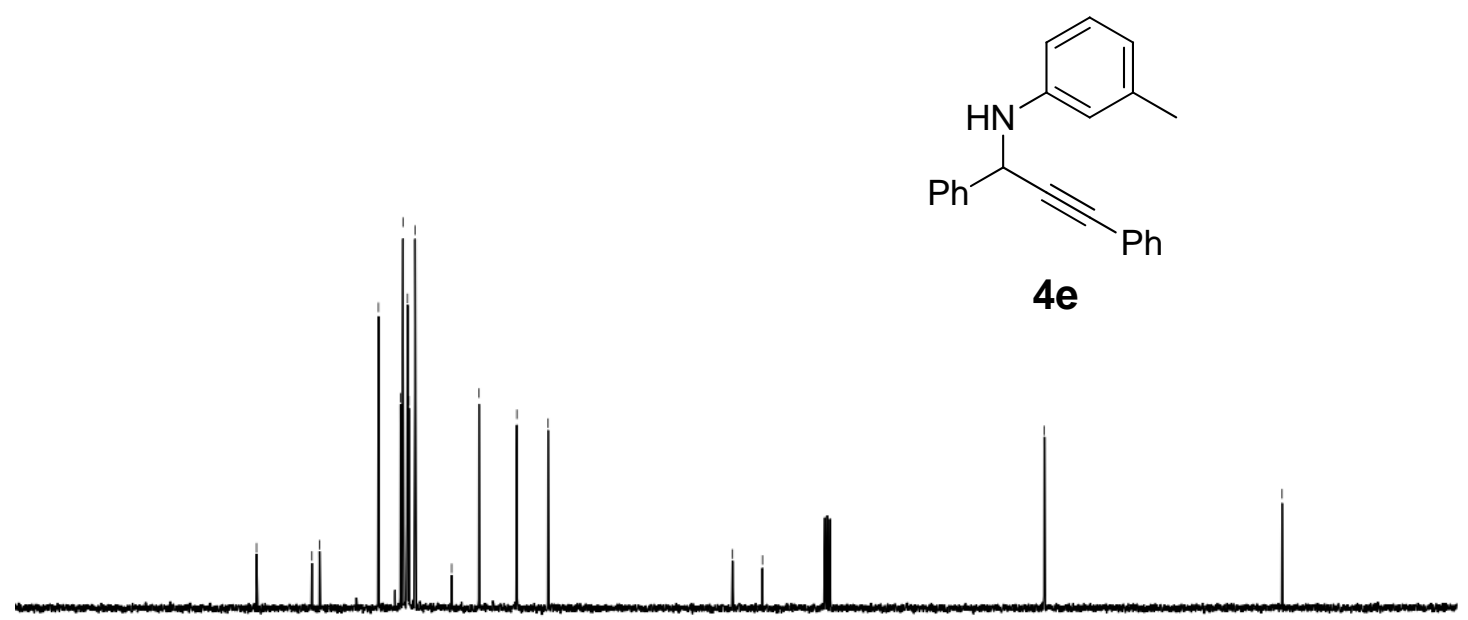

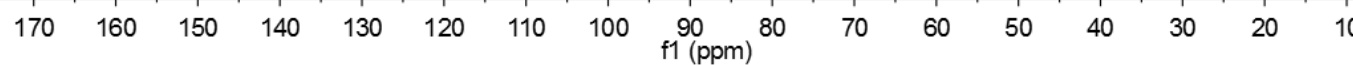

